\title{
Wachstum und Grenzflächenbeschaffenheit oxidischer Ausscheidungen in Silber
}

\author{
Dissertation \\ zur Erlangung des Doktorgrades \\ der Mathematisch-Naturwissenschaftlichen Fakultäten \\ der Georg-August-Universität Göttingen
}

vorgelegt von

Christian Kluthe

aus Büren 
D7

Referent:

Prof. Dr. Kirchheim

Korreferent:

PD Dr. Schneider

Tag der mündlichen Prüfung: $\quad$ 05.11.2003 
EINLEITUNG.

1 Materialphysikalischer Hintergrund 4

1.1 DIE INNERE OXIDATION BINÄRER LEGIERUNGEN ........................................ 4

1.1.1 Grundlagen der Inneren Oxidation.................................................... 4

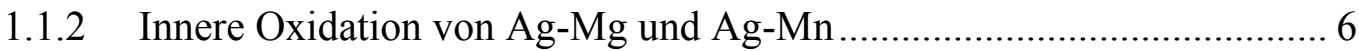

1.2 Die Metald/OXID (M/O)- GrenZFLÄCHE ............................................. 10

1.2.1 Modell der strukturellen Leerstellen ..................................................... 11

2 EXPeRIMENTELLE Methoden............................................... 14

2.1 FELDIONENMIKROSKOP UND TOMOGRAPHISCHE ATOMSONDE .................... 14

2.1.1 Das Feldionenmikroskop (FIM) .................................................... 14

2.1.2 Die tomographische Atomsonde (TAP) .............................................. 16

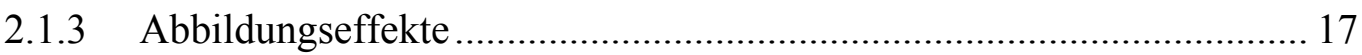

2.1.4 Auswerteverfahren der Atomsondendaten ......................................... 18

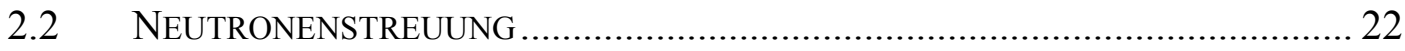

2.2.1 Grundlagen der Streuung mit Neutronen ............................................ 22

2.2.2 Prinzip der Neutronenkleinwinkelstreuung (SANS)............................ 25

2.2.3 Streuung an kugelsymmetrischen Teilchen ........................................ 27

2.2.4 Streuverhalten einer segregierten Schale H, D .................................... 29

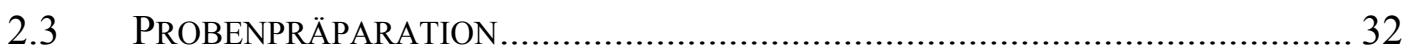

2.3.1 Präparation der innenoxidierten Proben für TAP- Analysen ................. 32

2.3.2 Präparation der SANS- Proben …..................................................... 33

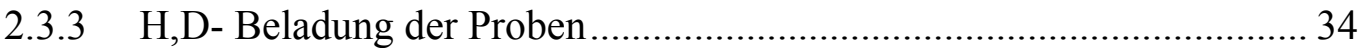

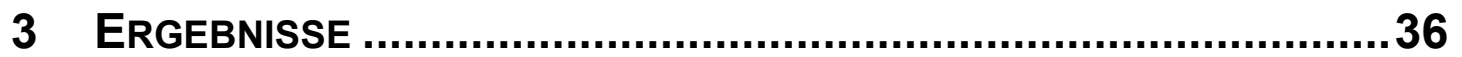

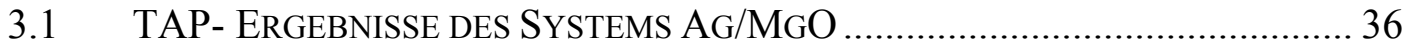

3.1.1 Innere Oxidation der AgMg- Legierung …........................................ 36

3.1.2 Untersuchung der Ag/MgO- Grenzfläche ........................................... 41

3.2 SANS- ERGEBNISSE DES SySTEMS AG/MGO ........................................ 44

3.2.1 Unbeladene Ag/MgO- Proben ............................................................... 44

3.2.2 H- und D- beladene $\mathrm{Ag} / \mathrm{MgO}-$ Proben ................................................. 49

3.2.3 Experimente an UHV- entgasten $\mathrm{Ag} / \mathrm{MgO}$ - Proben ............................. 53

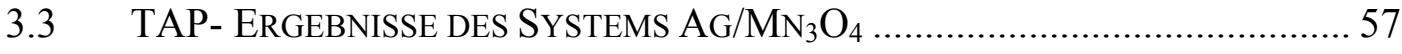

3.3.1 Innere Oxidation der AgMn- Legierung ............................................. 57 


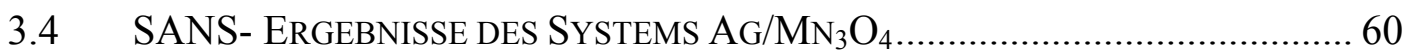

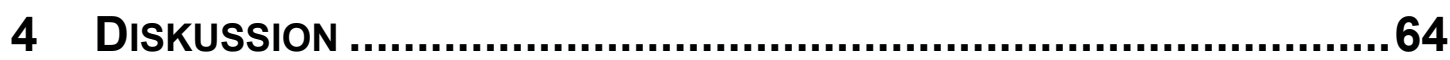

4.1 BILDUNGSKINETIK UND REIFUNG DER OXIDTEILCHEN ................................ 64

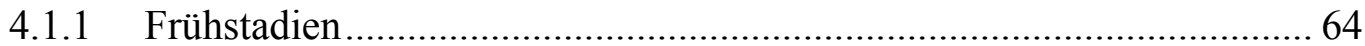

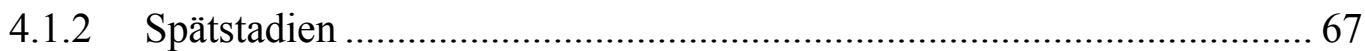

4.2 BeschafFENHEIT DER MEtAll/OxID (M/O)- GRENZFLÄChE..................... 72

4.2.1 Verifizierung des Kugel- Schale- Modells.......................................... 72

4.2.2 Segregation und Bindung von $\mathrm{O}$ und $\mathrm{H}$ an der Grenzfläche.................. 74

4.2.3 Änderung der Matrixdichte infolge der Segregation ............................ 76

4.2.4 Entwicklung der Grenzflächenbeschaffenheit ................................... 80

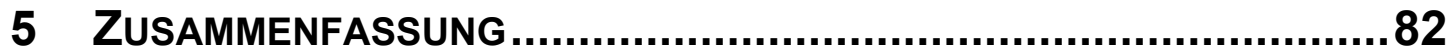

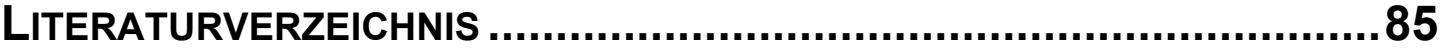

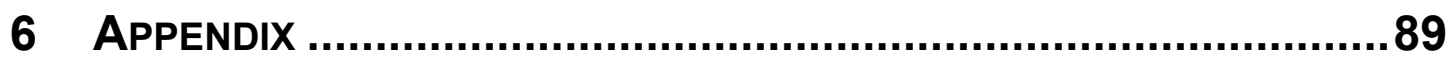

6.1 STREUQUERSCHNITT EINER SEGREGIERTEN SCHALE .................................. 89

6.2 Gesputterte Metald/OXID- Schichten................................................ 92

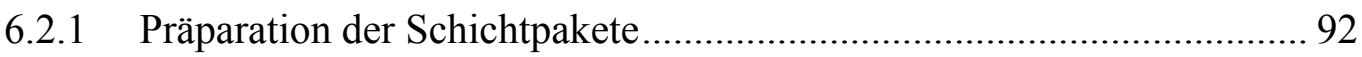

6.2.2 TAP- Analysen der Schichtpakete …................................................. 93 


\section{Einleitung}

Die Materialphysik leistet mit der Entwicklung neuer und verbesserter Werkstoffe einen wichtigen Beitrag für den technologischen Fortschritt auf unterschiedlichen Bereichen. Dabei versucht sie, den Zusammenhang zwischen mikroskopischem Gefüge und makroskopischen Eigenschaften zu ergründen und auf Grundlage dieses Wissens die Materialeigenschaften gezielt zu beeinflussen. Während in früheren Jahren die Erforschung metallischer Systeme im Vordergrund stand, sind heute vor allem Verbundmaterialien interessant, da in diesen die Eigenschaften unterschiedlicher Materialklassen verknüpft werden können. Dabei werden solche Materialien entwickelt, in denen metallische Phasen gemeinsam mit Polymeren, Karbiden, Nitriden oder Oxiden auf der Nanometerskala innerhalb des Gefüges vorliegen.

Für den Einsatz dieser Werkstoffe sind die mechanischen, elektrischen und magnetischen Eigenschaften der zugehörigen Grenzflächen entscheidend. Diese wichtigen Eigenschaften werden wiederum durch das Segregationsverhalten von Gasen, wie Sauerstoff oder Wasserstoff, an diesen Grenzflächen entscheidend beeinflußt. Ein sehr bekanntes Beispiel hierfür ist die sog. Wasserstoffversprödung hochfester Stähle mit kleinen Karbidausscheidungen. Die Segregation von Wasserstoff an den inneren Metall/Karbid- Grenzflächen geht mit der Ausbildung von Mikrorissen einher, die dann zu einem Sprödbruch des Werkstoffs führen [HuGGIN78]. Andererseits ist ein gutes Verständnis der Segregation von O und $\mathrm{H}$ an inneren Grenzflächen für die erfolgreiche Entwicklung nanostrukturierter Verbundmaterialen als Speichermedien für diese Gase unerläßlich. Wasserstoff kann ebenso auch als Sonde zur Charakterisierung des Gefüges dieser Materialien eingesetzt werden. Es ist daher von großem Interesse, den atomaren Aufbau solcher Grenzflächen und das Segregationsverhalten zu untersuchen.

Im Rahmen dieser Arbeit wird diese Fragestellung für Metall/Oxid (M/O)- Systeme behandelt. M/O- Grenzflächen treten in den Bereichen der Dispersionshärtung, der keramischen Schutzschichten, der Sensortechnik sowie in fast allen modernen Halbleiterbauelementen in Erscheinung. Da die Struktur der Grenzflächen stark von den bei der Herstellung vorhandenen Bedingungen abhängt, ist ein gutes Verständnis der ablaufenden Prozesse notwendig, um den Herstellungsprozeß gezielt zu kontrollieren. 
Im allgemeinen werden M/O- Grenzflächen durch die Deposition von Metallen auf oxidische Substrate hergestellt. Diese Art der Herstellung bedingt jedoch häufig Verunreinigungen, welche oft $\mathrm{zu}$ einer geringen Adhäsionsenergie des Metalls zum Oxid führen. Die im Rahmen dieser Arbeit untersuchten Systeme $\mathrm{Ag} / \mathrm{MgO}$ und $\mathrm{Ag} / \mathrm{Mn}_{3} \mathrm{O}_{4}$ wurden mit Hilfe der Methode der Inneren Oxidation (IO) hergestellt, durch welche nach kontrollierter Prozeßführung atomar scharfe M/O- Grenzflächen erzeugt werden. Ein weiterer Vorteil der IO ist, daß durch diese ein sehr hoher Anteil an M/O-Grenzflächen in einem kleinen Probenvolumen erzeugt werden kann (bis zu $100 \mathrm{~m}^{2}$ in einem $\mathrm{cm}^{3}$ der Ausgangslegierung).

Die durch Innere Oxidation von binären metallischen AgMg- und AgMn- Legierungen hergestellten, nanometergroßen $\mathrm{MgO}$ - und $\mathrm{Mn}_{3} \mathrm{O}_{4}$ - Ausscheidungen in $\mathrm{Ag}$ wurden mit Hilfe der tomographischen Atomsonde (TAP) und der Neutronenkleinwinkelstreuung (SANS) untersucht. Da die makroskopischen Eigenschaften durch die Struktur der Grenzflächen bestimmt sind, ist es erforderlich, diese mit Methoden höchster räumlicher Auflösung zu charakterisieren. Das TAP ermöglicht eine solch chemisch quantitative Untersuchung auf der Subnanometerskala und ist daher prädestiniert für die Untersuchung der Bildungs- und Wachstumskinetik der Oxidausscheidungen im Zuge der IO. Außerdem wird insbesondere das Segregationsverhalten von Wasserstoff an der M/OGrenzfläche untersucht. Ein detailliertes Verständnis dieses Segregationsverhaltens ermöglicht somit auch Rückschlüsse auf die Beschaffenheit der M/O- Grenzfläche. Der Wasserstoff wurde in Vorgängerarbeiten schon erfolgreich als Sonde zur Bestimmung von Defektdichten, Ausscheidungsbildung und Korngrößen eingesetzt [MAXELON01, KIRCHHEIM88, NoH96]. Um die Segregation von Wasserstoff und Deuterium an M/OTeilchengrenzflächen nachzuweisen, wird im Rahmen dieser Arbeit erstmals die Methode der Kleinwinkelneutronenstreuung (SANS) genutzt. Der Vorteil von SANS besteht insbesondere in dem großem Wirkungsquerschnitt des Wasserstoffs für Neutronen. Die unterschiedlichen Vorzeichen der kohärenten Streulängen von Wasserstoff und Deuterium für Neutronen ermöglichen es, durch Kontrastvariationsexperimente andere Streubeiträge auszuschließen und so die reine Segregation an den M/O- Grenzflächen zu untersuchen. Die optimale Untersuchung der betreffenden Systeme gelingt durch die Kombination von TAP und SANS. Mit Hilfe des TAP als Methode im Realraum können gezielt einzelne Teilchen und deren Grenzflächen analysiert werden. Untersu- 
chungen mit SANS als Methode im reziproken Raum liefern hingegen über sehr viele Teilchen gemittelte Aussagen, die einer hohen Zählstatistik unterliegen.

Die vorliegende Arbeit gliedert sich in vier Kapitel. Zunächst wird in Kapitel eins ein Überblick über die Grundlagen der Inneren Oxidation gegeben, welche dann auf die zu untersuchenden Systeme Ag-Mg und Ag-Mn angewendet werden. Des weiteren wird das Modell der strukturellen Leerstellen von Huang, Mader und Kirchheim [HuANG91] vorgestellt, welches die Struktur von $\{111\}$ - orientierten M/O- Grenzflächen beschreibt. Dieses Modell kann als Arbeitshypothese der folgenden Experimente angesehen werden. In Kapitel zwei werden die verwendeten experimentellen Methoden vorgestellt. Insbesondere wird das sog. Kugel- Schale Modell eingeführt, welches das Streuverhalten einer segregierten Schale H, D oder O beschreiben soll. Des weiteren wird die Präparation der TAP- und SANS- Proben, sowie deren Beladung mit Wasserstoff beschrieben. In Kapitel drei sind die Ergebnisse an innenoxidierten Ag-Mg- und AgMn- Proben aufgeführt. Der Schwerpunkt der Untersuchungen liegt hierbei auf dem System $\mathrm{Ag} / \mathrm{MgO}$. Für beide Systeme werden zunächst die TAP- Ergebnisse präsentiert, welche sich im wesentlichen auf die Bildungs- und Wachstumskinetik der oxidischen Ausscheidungen im Zuge der IO beziehen. Im Anschluß finden sich die Ergebnisse der SANS- Untersuchungen, bei denen die M/O- Grenzfläche im Vordergrund steht sowie deren Segregationsverhalten für H und D. In Kapitel vier werden zunächst die Ergebnisse zur Bildungs- und Wachstumskinetik der Oxidteilchen bewertet. Anschließend wird die Konsistenz der SANS- Ergebnisse überprüft und das Segregationsverhalten von $\mathrm{O}$ und $\mathrm{H}$ an der $\mathrm{M} / \mathrm{O}-$ Grenzfläche interpretiert. Abschließend wird die Grenzflächenbeschaffenheit für verschiedene Teilchenradien diskutiert und damit der Zusammenhang zur Wachstumskinetik der Oxidausscheidungen hergestellt. 


\section{Materialphysikalischer Hintergrund}

\subsection{Die Innere Oxidation binärer Legierungen}

Die Oxidation einer Legierung ist komplexer als die eines Reinmetalls, da die Legierungskomponenten aufgrund der verschiedenen chemischen Potentiale eine unterschiedliche Tendenz zur Oxidation aufweisen. Bei der Oxidation einer binären, homogenen Legierung $(\mathrm{AB})$ aus einem edlen Metall A und einem unedlen Metall B wird die Komponente B bevorzugt oxidiert. Ist ferner das Produkt aus Sauerstofflöslichkeit und Sauerstoffdiffusion sehr viel größer als das Produkt aus Konzentration

und Diffusion der B- Atome in der AB- Legierung $\left(c_{O} D_{O}>>c_{B}^{0} D_{B}\right)$, werden diese sogar innerhalb der Probe oxidiert. Der Sauerstoff diffundiert dann schneller in die Probe ein, als die Komponente B zum Rand. Es werden Ausscheidungen des Typs $\mathrm{B}_{\mathrm{m}} \mathrm{O}_{\mathrm{n}}$ innerhalb einer an B- Atomen verarmten Matrix gebildet. Dieser Fall wird als Innere Oxidation (IO) bezeichnet [SCHMALZRIED95]. Die Innere Oxidation kann aus thermodynamischer Sicht auch als Phasenseparation eines ternären A-B-O- Systems angesehen werden.

\subsubsection{Grundlagen der Inneren Oxidation}

Abb. 1.1 skizziert ein eindimensionales Modell zur Kinetik der Inneren Oxidation [WAGNER59]. Die Kurven entsprechen den Verläufen der O- und B-Konzentrationen ( $\mathrm{c}_{\mathrm{O}}$ und $\mathrm{c}_{\mathrm{B}}$ ) nahe der Reaktionsfront $\xi(\mathrm{t})$. Der Probenrand befindet sich im Koordinatenursprung, bei $x=0$. Die Diffusionsströme der beiden Reaktanden werden durch $\mathrm{j}_{0}$ und $\mathrm{j}_{\mathrm{B}}$ dargestellt. Links der Reaktionsfront $\xi(\mathrm{t})$ haben sich schon $\mathrm{B}_{\mathrm{m}} \mathrm{O}_{\mathrm{n}}$ - Ausscheidungen gebildet, rechts liegt die Ausgangslegierung AB vor (vgl. Abb. 1.1a). 
(a)

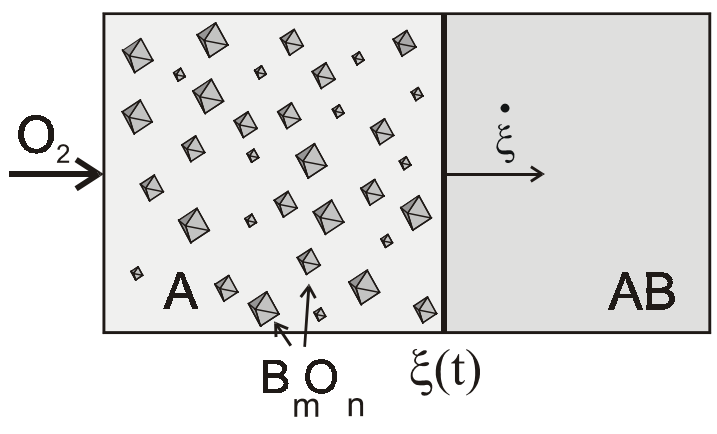

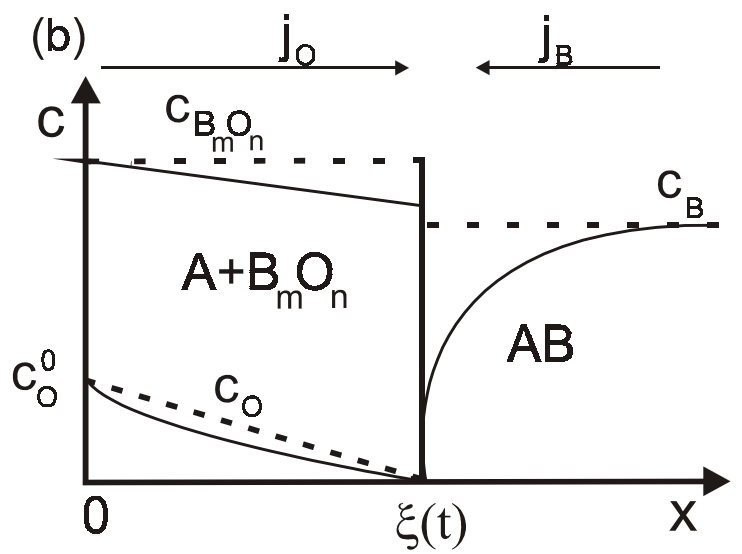

Abb. 1.1: $\quad$ Modell der Reaktionskinetik bei der IO einer Legierung AB (a) Skizze zur Veranschaulichung der Oxidationsfront; (b) Konzentrationsverläufe für den Fall starker (durchgezogene Linien) und zu vernachlässigender B-Gegendiffusion (gestrichelte Linien).

Der Fall schwacher B-Gegendiffusion $\left(c_{O} D_{O}>>c_{B}^{0} D_{B}\right)$ ist in Abb. 1.1b durch die gestrichelten Linien dargestellt. Die Sauerstoffkonzentration wird durch ein lineares Profil und die B-Konzentration durch ein Kastenprofil angenähert. Damit keine Oxidation der Komponente A stattfindet, darf die Sauerstoffkonzentration $\mathrm{c}_{\mathrm{O}}^{0}$ am Rand der Probe maximal gleich der Sättigungslöslichkeit $\mathrm{c}_{\mathrm{O}}^{\mathrm{s}}$ sein. Außerdem muß gewährleistet sein, daß von außen genügend Sauerstoff nachgeliefert werden kann, so daß $c_{O}^{0}$ an der Probenoberfläche stets konstant bleibt. Unter Vernachlässigung der äußeren Oxidation wandert dann die Reaktionsfront gemäß einem parabolischen Wachstumsgesetz durch die Probe [WAGNER59]:

$\xi(t)=\alpha \cdot \sqrt{D_{O} \cdot t}$

Dabei ist $\mathrm{D}_{\mathrm{O}}$ die Diffusionskonstante des Sauerstoffs. Der Faktor $\alpha$ kann unter Annahme geeigneter Randbedingungen aus den Diffusionsgleichungen für die eindimensionale Diffusion des Sauerstoffs und der Komponente B bestimmt werden, wie im Folgenden gezeigt wird. Betrachtet man nur den Fall schwacher B-Gegendiffusion, so ist der Sauerstofffluß $\mathrm{j}_{0}$ am Ort der Reaktionsfront $\xi$ durch das erste Ficksche Gesetz gegeben 
$\mathrm{j}_{\mathrm{O}}=-\mathrm{D}_{\mathrm{O}} \cdot \frac{\partial \mathrm{c}_{\mathrm{O}}}{\partial \mathrm{x}}=\frac{\mathrm{c}_{\mathrm{O}}^{0} \mathrm{D}_{\mathrm{O}}}{\xi(\mathrm{t})}$,

wobei mit $c_{O}=c_{O}^{0}-\frac{c_{0}^{0}}{\xi(t)} x$ für die Sauerstoffkonzentration ein lineares Profil angenommen wird. Die B-Atome werden als unbeweglich betrachtet $\left(\mathrm{c}_{\mathrm{B}}=\mathrm{c}_{\mathrm{B}}^{0}\right.$, für $\mathrm{x}>$ $\xi(\mathrm{t}))$. Während eines Zeitintervalls dt wird eine Schicht der Dicke $\mathrm{d} \xi$ durchwandert. Im Bezugssystem der wandernden Reaktionsfront müssen (am Ort $x=\xi(t)$ ) die Sauerstoffatome und die B-Atome der Stöchiometrie $\beta=m / n$ des Oxids $\mathrm{B}_{\mathrm{n}} \mathrm{O}_{\mathrm{m}}$ entsprechen:

$\beta \cdot c_{B} \cdot d \xi=c_{O}^{0} \frac{D_{O}}{\xi} \cdot d t$

Die Integration dieses Ausdrucks von $\mathrm{t}^{\prime}=0$ bis $\mathrm{t}$ und $\xi^{\prime}=0$ bis $\xi$ liefert mit Hilfe von (1.1) und (1.2) die momentane Lage der Reaktionsfront:

$$
\Rightarrow \quad \xi(\mathrm{t})=\sqrt{\frac{2 \mathrm{c}_{\mathrm{O}}^{0}}{\beta \cdot \mathrm{c}_{\mathrm{B}}} \mathrm{D}_{0} \cdot \mathrm{t}}
$$

Gleichung (1.4) gilt für den Fall einer zu vernachlässigenden B- Gegendiffusion. Bei gegebener Probendicke läßt sich damit auch die Zeit ausrechnen, nach der die Oxidationsfront die Probe komplett durchwandert hat. Weiterführende Betrachtungen der Grundlagen der Inneren Oxidation sind in [WAGNER59, GEGNER95, SCHMALZRIED95] zu finden.

\subsubsection{Innere Oxidation von Ag-Mg und Ag-Mn}

In Anlehnung an Kap. 1.1.1 wird zunächst die Gültigkeit der Voraussetzungen für die Innere Oxidation der in dieser Arbeit zu untersuchenden Systeme $\mathrm{Ag} / \mathrm{MgO}$ und $\mathrm{Ag} / \mathrm{Mn}_{3} \mathrm{O}_{4}$ überprüft. Die Sauerstoffdiffusion im Grundmetall Silber ist sehr hoch, seine Löslichkeit bei niedrigen Temperaturen hingegen gering [RAMANARA72]: 


$$
\begin{aligned}
& \mathrm{D}_{\mathrm{O}}(\mathrm{Ag})=4,9 \cdot 10^{-3} \exp \left(\frac{-48,567 \mathrm{~kJ}}{\mathrm{RT}}\right)\left[\frac{\mathrm{cm}^{2}}{\mathrm{~s}}\right] \quad(\mathrm{T}=1023 \text { bis } 1223 \mathrm{~K}) . \\
& \mathrm{c}_{\mathrm{O}}^{\mathrm{s}}(\mathrm{Ag})=7,2 \cdot \exp \left(\frac{-48,148 \mathrm{~kJ}}{\mathrm{RT}}\right) \quad[\text { At. } \%] \quad(\mathrm{T}=1023 \text { bis } 1223 \mathrm{~K}) .
\end{aligned}
$$

Nach Adda und Philibert [ADDA66] gilt für die Magnesiumdiffusion im Silber:

$$
\mathrm{D}_{\mathrm{Mg}}(\mathrm{Ag})=1,76 \cdot 10^{-3} \exp \left(\frac{-140,9 \mathrm{~kJ}}{\mathrm{RT}}\right)\left[\frac{\mathrm{cm}^{2}}{\mathrm{~s}}\right] .
$$

Die Diffusion von Mangan in Silber ist im Bereich von $500-800{ }^{\circ} \mathrm{C}$ etwa gleich der von Magnesium und beträgt [MAKUTA79]:

$$
\mathrm{D}_{\mathrm{Mn}}(\mathrm{Ag})=4,29 \cdot \exp \left(\frac{-196 \mathrm{~kJ}}{\mathrm{RT}}\right)\left[\frac{\mathrm{cm}^{2}}{\mathrm{~s}}\right] \quad(\mathrm{T}=883 \text { bis } 1212 \mathrm{~K})
$$

Der Diffusionskoeffizient von Magnesium und Mangan ist somit um fünf bis sechs Größenordnungen kleiner als der des Sauerstoffs in Silber (vgl. Abb. 1.2), wodurch die Ungleichung $c_{O} \cdot D_{O}>c_{B} \cdot D_{B}$ für beide $\mathrm{zu}$ untersuchenden Systeme erfüllt ist, da die Konzentrationen sich nur um zwei Größenordnungen unterscheiden.

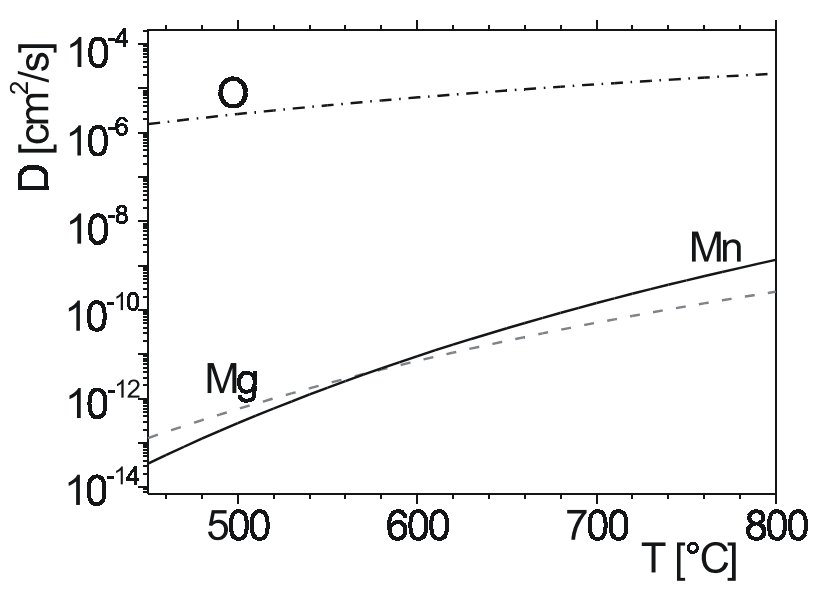

Abb. 1.2: $\quad$ Temperaturabhängikeit der Diffusionskoeffizienten von $\mathrm{O}, \mathrm{Mn}$ und $\mathrm{Mg}$ in $\mathrm{Ag}$.

Nach Böhm und Kahlweit nimmt die Teilchenzahldichte im Verlauf der Inneren Oxidation mit steigender Oxidationstiefe ab [BöHM64]. Dies liegt an einer Gegendiffusion der unedleren Legierungskomponente, da die B- Atome mit zunehmender 
Probentiefe mehr Zeit für eine gerichtete Diffusionsbewegung haben. Bei Proben von mehreren mm Dicke werden daher in der Mitte der Probe größere Ausscheidungen beobachtet, als in der Nähe der Probenoberfläche [BöHM64]. Für die zu untersuchenden Proben von 150- $200 \mu \mathrm{m}$ Dicke ist diesem Effekt allerdings, auch aufgrund der hohen Beweglichkeit von Sauerstoff im Grundmetall Silber, nur eine geringe Bedeutung zu zurechnen. Durch das elektrolytische Dünnen wird zudem jede FIM- Spitze immer aus der Mitte der zylinderförmigen Rohlinge präpariert (siehe Kap. 2.3.1). Diese Art der Präparation gewährleistet deshalb die systematische Untersuchung der Teilchenzahldichten sowie der Radien der gebildeten Ausscheidungen der jeweiligen Zustände.

Der ausgeschiedenen Phase kann man einen zugehörigen kritischen Keimradius

$$
\mathrm{r}_{\text {krit }}=\frac{2 \gamma}{\Delta \mathrm{G}_{\mathrm{V}}}
$$

zuordnen [SHEWMON69], wobei $\gamma$ die Grenzflächenenergie und $\Delta \mathrm{G}_{\mathrm{V}}$ den Gewinn an freier Energie pro Volumeneinheit bezeichnet. Sowohl Mg als auch Mn weisen sehr hohe Beträge der Gibbsche Bildungsenthalpien der jeweiligen Oxide auf. Der Gewinn an freier Energie ist bei der Bildung von $\mathrm{Mn}_{3} \mathrm{O}_{4}$ etwas geringer als für $\mathrm{MgO}$ (siehe Abb. $1.3)$.

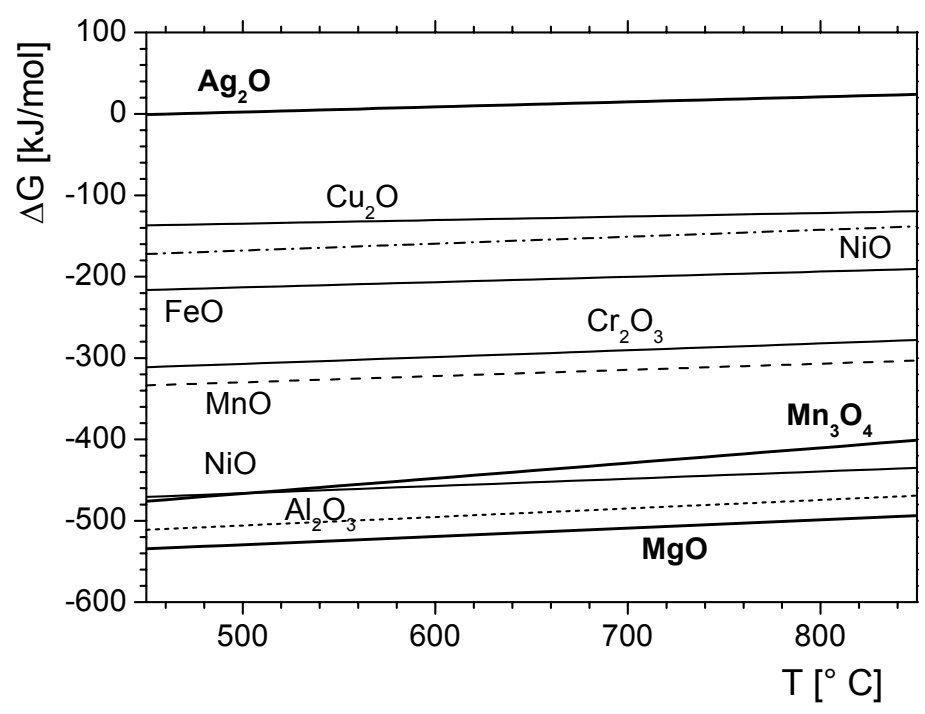

Abb. 1.3: Bildungsenthalpien verschiedener Oxide nach [FROMM76]. 
Für die Legierung Ag-1At.\% $\%$ gg wird bei $\mathrm{T}=750{ }^{\circ} \mathrm{C}$ dieser kritische Keimradius so klein, daß jedes MgO- Molekül für sich schon einen stabilen Keim darstellt ${ }^{1}$. Für $\mathrm{Mn}_{3} \mathrm{O}_{4}$ berechnet sich bei der gleichen Temperatur ein kritischer Keimradius ${ }^{2}$ von 0,5 nm. Bei gleicher Oxidationstemperatur sollten sich daher bei der IO einer Ag-0,4At.\%MnLegierung größere Ausscheidungen bilden, als bei IO einer Ag-0,4At.\%Mg- Legierung.

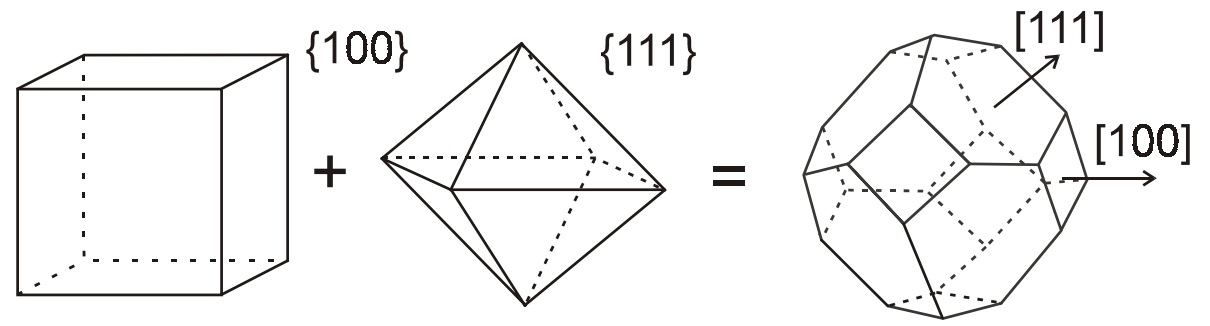

Abb. 1.4: Schematische Konstruktion der 14-Flächler als Gemisch aus \{100\}- und \{111\}-Ebenen [GEGNER95].

Im idealisierten Fall sind die sich bildenden Ausscheidungen kugelförmig, da die Kugelform ein Minimum der Oberfläche für ein gegebenes Volumen darstellt. Oft werden jedoch Teilchenformen abweichend von der Kugelform beobachtet, was durch eine Anisotropie von Verzerrungs- oder Grenzflächenenergie erklärt wird. Der Misfit der kohärenten $\mathrm{Ag} / \mathrm{MgO}$ - Grenzfläche fällt mit 3,1\% allerdings sehr klein aus und ist isotrop in jede Richtung. Die Verzerrungsenergie ist daher nicht die bestimmende Größe für die Teilchenmorphologie dieses Systems [MADER89]. Für Ag nimmt die $\{111\}$ - orientierte Oberfläche das Minimum der Grenzflächenenergie ein [VITOS98]. Es ist daher anzunehmen, daß auch die $\{111\}$ - orientierte $\mathrm{Ag} / \mathrm{MgO}$ - Grenzfläche eine geringere Energie aufweist, als die $\{100\}$ - und $\{110\}$ - Grenzfläche. Die Morphologie der MgO- Teilchen (mit NaCl- Struktur) wird also durch die Anisotropie der Grenzflächenenergie bestimmt. Dennoch bilden sich in Ag nicht perfekte Oktaeder, sondern an den [100]- Richtungen abgestumpfte Oktaeder aus $\{111\}$ - und $\{100\}$ - Flächen (siehe Abb. 1.4,). Das Abstumpfen der Ausscheidungen in [100]- Richtung kann dabei als Streben in Richtung der idealen Kugelform interpretiert werden.

\footnotetext{
${ }^{1}$ Mit $\gamma=2 \cdot 10^{-4} \mathrm{~J} / \mathrm{cm}^{2}, \Delta \mathrm{G}_{\mathrm{MgO}}=5,03 \cdot 10^{5} \mathrm{~J} / \mathrm{mol}, \mathrm{V}_{\mathrm{MgO}}=11,25 \mathrm{~cm}^{3} / \mathrm{mol}: \mathrm{r}_{\text {krit. }}=0,9 \AA(\operatorname{vgl~r} \mathrm{MgO}=1,65 \AA)$.

${ }^{2}$ Unter Annahme einer, zum System Ag/MgO ähnlichen Grenzflächenenergie u. $\mathrm{V}_{\mathrm{Mn} 3 \mathrm{O} 4}=79,45 \mathrm{~cm}^{3} / \mathrm{mol}$.
} 
$\mathrm{Mn}_{3} \mathrm{O}_{4^{-}}$Ausscheidungen besitzen eine tetragonale Spinell- Struktur und bilden eine semikohärente Grenzfläche zu Ag aus [KooI00]. Der Misfit beträgt im Mittel 4,6 \% und ist nicht isotrop in alle Raumrichtungen. Die a- Achse des tetragonalen Oxids ist mit a = 5,67 $\AA$ im Bereich der Gitterkonstante der meisten fcc- Metalle, die c- Achse hingegen ist wesentlich länger ( $c=9,44 \AA$ ). Ein Minimum der Misfitenergie wäre für plattenförmige Ausscheidungen mit $\{100\}$ - Grenzflächen und der c- Achse als Normalen der Habitusebene der Platten gewährleistet. Bei Berücksichtigung von Misfit- und Grenzflächenenergie sind für dieses System oktaedrische $\mathrm{Mn}_{3} \mathrm{O}_{4}$ - Ausscheidungen mit $\{100\}$ Grenzflächen zu erwarten. Tatsächlich werden nach der Inneren Oxidation nur Ausscheidungen mit $\{111\}$ - Facetten beobachtet. Interessanterweise ändert sich diese Morphologie der Ausscheidungen nach einer Hochtemperaturglühung im Vakuum. Nach Vakuumglühung der $\mathrm{Ag} / \mathrm{Mn}_{3} \mathrm{O}_{4}$ - Proben bei $650{ }^{\circ} \mathrm{C}$ für eine Woche haben sich aus den $\{111\}$ - facettierten Oktaedern Kuboktaeder, also in den $\{100\}$ - Richtungen abgestumpfte Oktaeder, analog zu MgO- Ausscheidungen in Ag (siehe Abb. 1.4), umgebildet [KoOI00].

\subsection{Die Metall/Oxid (M/O)- Grenzfläche}

Es ist bemerkenswert, daß $\{111\}$ - Grenzflächen sowohl für $\mathrm{MgO}$ - als auch für $\mathrm{Mn}_{3} \mathrm{O}_{4}$ Ausscheidungen in Ag stabil sind. Da die $\{111\}$-Ebenen des Oxids abwechselnd mit Mg- und O-Ionen belegt sind, stellen diese polare Flächen dar. In der terminierenden $\{111\}$-Ebene des Oxids zur Ag-Matrix entsteht daher ein resultierendes Dipolmoment. Eine geladene Oberfläche bedeutet jedoch eine nahezu unendliche Grenzflächenenergie. Bei einer nur halb besetzten terminierenden $\{111\}$-Fläche aus Sauerstoffatomen (siehe Kap. 1.2.1), wie sie Huang, Mader und Kirchheim vorschlagen, verschwindet dieser divergierende Energiebeitrag [HUANG91]. Die Grenzflächenenergie dieser Ebenen wird daher so gering, daß ein Facettieren der Teilchen in dieser kristallographischen Richtung energetisch günstig erscheint [MADER89], wie im folgenden Modell erläutert. 


\subsubsection{Modell der strukturellen Leerstellen}

An verschiedenen durch Innere Oxidation hergestellten Systemen, wie zum Beispiel $\mathrm{Ag} / \mathrm{CdO}$ und $\mathrm{Nb} / \mathrm{Al}_{2} \mathrm{O}_{3}$ [MADER89], wurden die mit hochauflösender Elektronenmikroskopie beobachteten Strukturen der Metall/Oxid (M/O)-Grenzflächen mit Bildsimulationen verglichen. Aus den zugehörigen Ergebnissen schlußfolgerten die Autoren, daß die äußere Atomlage des Oxids zum Metall ausschließlich aus O- Atomen besteht. Es wird angenommen, daß dies für alle $\mathrm{M} / \mathrm{O}-$ Grenzflächen der Fall ist [MADER89].

In Wasserstoffbeladungsexperimenten [HUANG88] beobachtete man nach einer Glühung der innenoxidierten Probe bei hohen $\mathrm{O}_{2}$-Partialdrücken reversibel sowie irreversibel haftenden Wasserstoff. Nach einer Glühung im Vakuum findet man hingegen nur reversibel haftenden Wasserstoff. Dieses Segregationsverhalten des Wasserstoffs an der Grenzfläche kann mit Hilfe des folgenden Modells erklärt werden (Abb. 1.5):

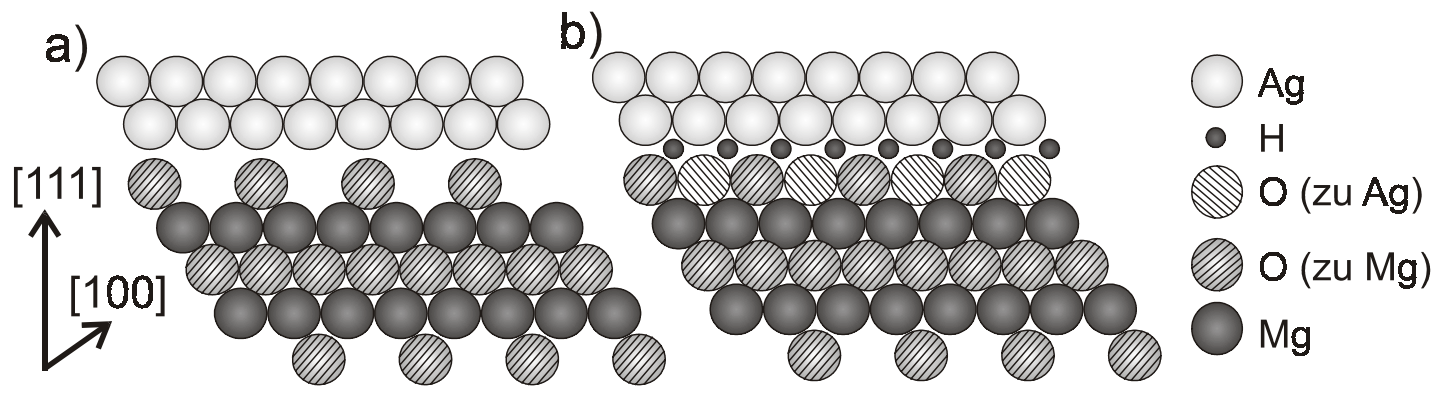

Abb. 1.5: $\quad$ Modell zur Segregation von Exzeß-O und Wasserstoff an $\{111\}-M / O-$ Grenzflächen; Bsp. Ag/MgO a) Strukturelle Leerstellen der halb besetzten, terminierenden $O$-Ebene des Oxids, b) Exzeß-O in den strukturellen Leerstellen, sowie irreversibel gebundener Wasserstoff an der M/O-Grenzfläche.

Unter der Annahme, daß die MgO- Ausscheidungen eine perfekte Stöchiometrie besitzen und nach außen elektrisch neutral sind, darf eine abschließende $\mathrm{O}^{2-}$-Lage nur zur Hälfte besetzt sein [HUANG91]. Die andere Hälfte stellen strukturelle Leerstellen dar. Letztere werden bei ausreichend hohen $\mathrm{O}_{2}$ - Drücken (und Temperaturen) mit sogenanntem Exzeß- Sauerstoff besetzt, welcher an der Grenzfläche eine schwache Bindung zu den Matrixatomen eingeht. Bei einer H- Beladung des Systems kann dieser Exzeß- O dann Wasserstoff durch O-H- Bindungen irreversibel an sich binden. Die zum Oxid gehörenden Sauerstoffatome tragen hingegen nicht $\mathrm{zu}$ dieser irreversiblen 
Bindung bei. Durch eine Bestimmung des in der Probe verbliebenen Wasserstoffs hat man somit eine Methode gefunden, die obige Vorstellung einer M/O- Grenzfläche zu verifizieren.

Die Verwendung der Bezeichnungen „Exzeß-O“ und „Oxid-O“ innerhalb des strukturellen Modells erweckt den Eindruck, daß es sich um unterschiedlich gebundene Sauerstoffatome an der Grenzfläche handelt. Tatsächlich geht der segregierte Sauerstoff allerdings eine Art Mischzustand mit dem zum Oxid gebundenen Sauerstoff ein und ist von diesem nicht zu unterscheiden [GEGNER95]. Ein an der M/O- Grenzfläche segregierter Wasserstoff wird von diesen ambivalenten Sauerstoff dann als O-H irreversibel gebunden. Im Rahmen dieser Arbeit wird der Begriff „Exzeß- O“ allerdings dennoch verwendet, da er die quantitative Auswertung der Experimente vereinfacht. Hier ist der Exzeß-O schlicht als die Menge des Sauerstoffs anzusehen, die zusätzlich zur Stoichiometrie des Oxids an der Teilchengrenzfläche segregiert.

Aus dem strukturellen Modell von Huang, Mader und Kirchheim lassen sich die theoretischen Belegungsdichten von Exzeß- O und Wasserstoff für die zu untersuchenden Systeme abschätzen. Für eine allein aus O- Atomen terminierte $\{111\}$ - Grenzfläche des Oxids berechnen sich:

$\Theta_{\mathrm{O}}^{\{111\}}(\mathrm{MgO})=1,30 \cdot 10^{15} \mathrm{~cm}^{-2} \quad$ und $\quad \Theta_{\mathrm{O}}^{\{111\}}\left(\mathrm{Mn}_{3} \mathrm{O}_{4}\right)=1,08 \cdot 10^{15} \mathrm{~cm}^{-2}$.

Nach dem strukturellen Modell kann die Hälfte dieses Sauerstoffs als Exzeß- O angesehen werden:

Für MgO: $\quad \Theta_{\text {Ex.O }}\{111\}=6,5 \cdot 10^{14} \mathrm{~cm}^{-2}$

Für $\mathrm{Mn}_{3} \mathrm{O}_{4}: \quad \Theta_{\text {Ex.O }}\{111\}=5,4 \cdot 10^{14} \mathrm{~cm}^{-2}$

Schließlich muß noch die Teilchenmorphologie berücksichtigt werden. $\mathrm{MgO}$ - Teilchen in Ag haben die Form von abgestumpften Oktaedern mit $\{111\}$ - und $\{100\}$ - Facetten [GEGNER95]. Die $\{100\}$ - Grenzflächen bestehen sowohl aus Mg, als auch aus O- Ionen. Somit ist an der terminierenden $\{100\}$ - Grenzfläche kein Exzeß- O vorhanden $\left(\Theta_{\mathrm{ExO}}\{100\}=0\right) . \mathrm{Mn}_{3} \mathrm{O}_{4}$ scheidet sich in $\mathrm{Ag}$ in Form von Oktaedern aus, welche nur aus 
$\{111\}$ - Grenzflächen bestehen [KoOI00]. Auf ein gesamtes Teilchen bezogen, betragen die theoretischen Exzeß- O Belegungsdichten demnach:

Für $\mathrm{MgO}^{3}: \quad \Theta_{\text {Ex.O }}$ theor. $=0,5 \cdot \Theta_{\text {Ex. }}{ }^{\{111\}}=3,25 \cdot 10^{14} \mathrm{~cm}^{-2}$

Für $\mathrm{Mn}_{3} \mathrm{O}_{4}: \quad \Theta_{\text {Ex.O }}{ }^{\text {theor. }}=\Theta_{\text {Ex.O }}\{111\}=5,4 \cdot 10^{14} \mathrm{~cm}^{-2}$.

Für den Fall einer Bildung von $\mathrm{H}_{2} \mathrm{O}$ an der Grenzfläche sind entsprechend die doppelten Mengen für die theoretischen $\mathrm{H}-$ Belegungsdichten zu erwarten:

Für $\mathrm{MgO}: \quad \Theta_{\mathrm{H}}{ }^{\text {theor. }}=2 \cdot \Theta_{\text {Ex.O }}{ }^{\text {theor }}=6,5 \cdot 10^{14} \mathrm{~cm}^{-2}$

Für $\mathrm{Mn}_{3} \mathrm{O}_{4}: \quad \Theta_{\mathrm{H}}^{\text {theor. }}=2 \cdot \Theta_{\text {Ex.O }}{ }^{\text {theor }}=10,8 \cdot 10^{14} \mathrm{~cm}^{-2}$.

Mit Hilfe von Elektronen-Energie-Verlust-Spektroskopie (EELS) an den Systemen $\mathrm{Ag} / \mathrm{MgO}$ und $\mathrm{Cu} / \mathrm{MgO}$ wurde bereits der qualitative Nachweis für die Segregation von Exzeß- O an der M/O- Grenzfläche erbracht [BACKhaus00, BackHaus99, PiPPEL00]. Nach Untersuchungen solcher Grenzflächen mit APFIM [JANG92, CHAN95, ORTH96] soll in dieser Arbeit die M/O- Grenzfläche sowohl mit Hilfe von TAP als auch mit SANS untersucht werden. Hierbei wird im Speziellen das Segregationsverhalten von $\mathrm{H}$ (und D) an den M/O- Grenzflächen studiert. Nach obiger Vorstellung ist anzunehmen, daß unter $\mathrm{H}-$ Angebot der schwach gebundene Exzeß- $\mathrm{O}$ zu $\mathrm{H}_{2} \mathrm{O}$ reagiert. Der $\mathrm{H}$ kann somit als Sonde zur Charakterisierung der M/O- Grenzfläche benutzt werden, da die segregierte Menge an $\mathrm{H}$ direkt an die Belegungsdichte des Exzeß- $\mathrm{O}$ an der M/OGrenzfläche gekoppelt ist.

\footnotetext{
${ }^{3}$ Bei einem Flächenverhältnis $\mathrm{A}^{\{111\}} / \mathrm{A}^{\{100\}}$ des MgO- Teilchens von 1:1.
} 


\section{Experimentelle Methoden}

In diesem Kapitel wird die Funktionsweise der tomographischen Atomsonde, eine Kombination aus Feldionenmikroskop und positionssensitivem Detektor (PSD), erklärt. Die für die Arbeit relevanten Auswerteverfahren werden geschildert. Ausführlichere Informationen über die Feldionenmikroskopie sind in [MILLER96] zu finden.

\subsection{Feldionenmikroskop und tomographische Atomsonde}

\subsubsection{Das Feldionenmikroskop (FIM)}

Als Probe für das Feldionenmikroskop (FIM) dient eine elektrolytisch gedünnte Spitze mit einem Krümmungsradius von 10 bis $50 \mathrm{~nm}$. Diese wird in eine Hauptvakuumkammer mit $10^{-7}$ bis $10^{-8}$ Pa Restgasdruck geschleust und dort in einen beweglichen Kupferhalter eingebaut. In einem Abstand von $\mathrm{R}=50 \mathrm{~mm}$ befinden sich zwei hintereinandergeschaltete Kanalplatten, die als Signalverstärker dienen (im FIMModus) und ein Bildschirm (siehe Abb. 2.1).

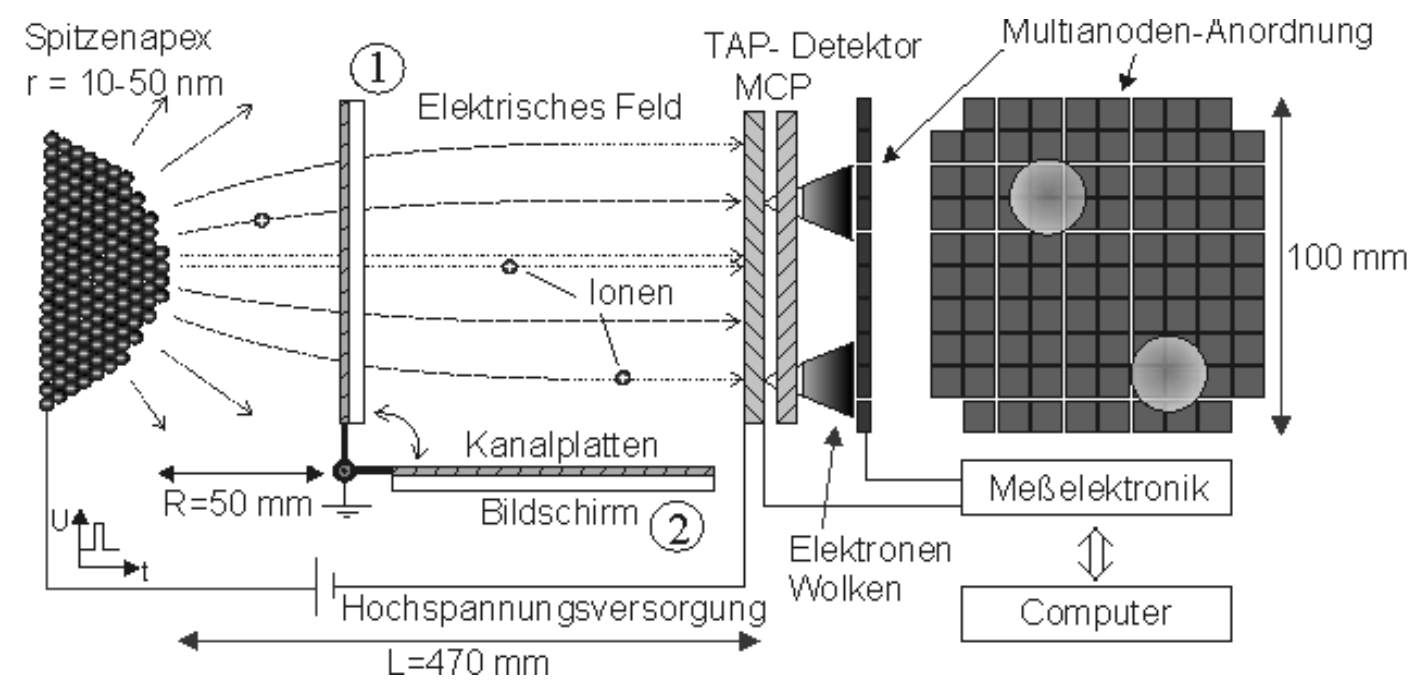

Abb. 2.1: Feldionenmikroskop mit 3-dimensionalem Detektor; (1) FIM-Modus (2) 3dAtomsonden-Modus 
In der Hauptkammer wird ein Druck von $5 \cdot 10^{-3}$ Pa Bildgas $\left(\mathrm{He}, \mathrm{Ne}\right.$ oder $\left.\mathrm{H}_{2}\right)$ eingestellt. Die Spitze wird dann auf ein positives Potential der Stärke U=2-20 kV DC gelegt, so daß zwischen ihr und dem geerdeten Bildschirm ein elektrisches Feld der Stärke

$\mathrm{F}=\frac{\mathrm{U}}{\mathrm{k}_{\mathrm{f}} \cdot \mathrm{r}_{\mathrm{t}}}$

(bis $50 \frac{\mathrm{v}}{\mathrm{nm}}$ ) entsteht. Der Geometriefaktor $\mathrm{k}_{\mathrm{f}}=5$ bis 7 ist von der Spitzenform abhängig, $r_{t}$ ist der mittlere Krümmungsradius der Spitze. Die Bildgasatome werden in diesem Feld polarisiert und zum Spitzenapex angezogen, wo sie thermalisiert werden, d.h. ihre kinetische Energie durch inelastische Stöße abgeben. Bei ausreichender Feldstärke erfolgt dann die Ionisation der Bildgasatome unter einem kritischen Abstand vom Apex per Tunnelprozeß [MüLlER60]. Die Bildgasionen werden radial in Richtung der Kanalplatten beschleunigt. Beim Auftreffen der Bildgasionen auf der vorderen Seite der Kanalplatten werden Elektronenlawinen, sog. Szintillationen, ausgelöst, die auf dem Bildschirm als helle Leuchtpunkte erscheinen. Um die Beweglichkeit der Bildgasatome an der Oberfläche zu vermindern, muß die Spitze auf etwa $25 \mathrm{~K}$ abgekühlt werden. Die Spitzenoberfläche wird in dieser atomistischen Betrachtung aus diskreten Atomlagen verschiedener Richtungen aufgebaut, so daß die Feldstärke entlang der Oberfläche schwankt. Die Terrassenstufen ionisieren am stärksten, erscheinen somit am hellsten. Netzebenen werden deshalb als konzentrische Ringe sichtbar. Die lokal unterschiedliche Krümmung des Apex führt zu verschiedener Vergrößerung einzelner Bereiche der Spitzenoberfläche. Gebiete stärkerer Krümmung erscheinen heller als Gebiete schwächerer Krümmung. Bei weiterer Steigerung der angelegten Spannung kann das elektrische Feld so groß werden, daß Oberflächenatome abgelöst werden. Diesen Vorgang bezeichnet man als Feldverdampfung. Die Oberflächenatome werden dabei Ebene für Ebene abgetragen. Aufgrund des endlichen Schaftwinkels der Spitze nimmt der Krümmungsradius mit fortlaufender Feldverdampfung immer weiter zu. Gleichzeitig wird dann die Vergrößerung geringer. 


\subsubsection{Die tomographische Atomsonde (TAP)}

Abb. 2.1 skizziert das FIM kombiniert mit einem ortsauflösenden Detektor. Um in den TAP-Modus zu wechseln, wird das Abbildungssystem des FIM weggeklappt. Anschließend wird die Bildgaszufuhr unterbrochen und das Bildgas entfernt. Überlagert man zur Grundspannung $U_{0}$ eine Pulspannung $U_{p}$ von 15 bis $25 \%$ der Grundspannung, feldverdampfen einzelne Atome der Spitzenoberfläche. Die chemische Identifizierung der detektierten Ionen erfolgt mittels Flugzeitmassenspektrometrie. Jeder Hochspannungspuls startet eine Zeitmessung, welche beim Auftreffen eines feldverdampften Ions auf der Mikrokanalplatte wieder gestoppt wird. Unter Berücksichtigung der Energieerhaltung läßt sich dann mit der Flugzeit $\mathrm{t}$ die spezifische Masse m/q des detektierten Ions bestimmen:

$$
\frac{\mathrm{m}}{\mathrm{q}}=2 \mathrm{e}\left(\mathrm{U}_{0}+\mathrm{U}_{\mathrm{P}}\right) \frac{\mathrm{t}^{2}}{\mathrm{~L}^{2}}
$$

Mit Hilfe des positionssensitiven Detektors läßt sich zudem der Ursprungsort eines detektierten Ions in der Probe mit Hilfe einer Punktprojektion berechnen. Die laterale Atomposition wird im wesentlichen durch Berechnung des Ladungsschwerpunktes, der auf der Multianode auftreffenden Elektronenwolke, bestimmt. Die Tiefenkoordinate z wird aus der Anzahl der feldverdampften Atomlagen, d.h. aus der Anzahl der detektierten Ionen $\mathrm{N}$, berechnet. Mit Kenntnis des Molvolumens $v_{\mathrm{a}}$ kann man aus dem elementaren Meßvolumen $\Delta \mathrm{V}$ direkt auf die zugehörige Tiefe $\Delta \mathrm{z}$ schließen:

$$
\Delta \mathrm{z}=\frac{\Delta \mathrm{N} \cdot \mathrm{v}_{\mathrm{a}}}{\mathrm{S}_{\mathrm{D}} \cdot \mathrm{d}_{\mathrm{a}}^{2}}
$$

$\mathrm{d}_{\mathrm{a}}=\mathrm{d} / \mathrm{M}$ ist die auf den Detektor projizierte Probenfläche, $\mathrm{S}_{\mathrm{D}} \approx 0,5$ die Detektoreffizienz. Aufgrund der Beschaffenheit der Mikrokanalplatten werden maximal nur 50\% aller feldverdampften Ionen detektiert. Mit fortlaufender Feldverdampfung wird, wie in Kap. 2.1.1 beschrieben, die Vergrößerung geringer. Der Analysebereich wird somit im Verlauf einer Messung immer größer. Infolgedessen werden $\Delta z$ und $d_{a}$ sukzessiv 
bestimmt. Die absolute Tiefe $\mathrm{z}$ erhält man durch numerische Integration über die elementare Tiefe $\Delta z$.

\subsubsection{Abbildungseffekte}

In phasenseparierten Proben enthält der Bereich der Spitze zwei Phasen unterschiedlicher Feldverdampfungsfeldstärken, häufig nahezu kugelförmige Ausscheidungen in einer Matrix. Bei stationärer Feldverdampfung müssen über beiden entmischten Phasen die jeweiligen Feldverdampfungsfeldstärken erreicht sein. Dies ist nach Gl. (2.1) nur durch eine sich lokal einstellende Variation des Krümmungsradius möglich. Die Feldionisationswahrscheinlichkeit eines Bildgasatoms ist in Bereichen kleinerer Krümmung mit geringer Feldstärke kleiner, als in Bereichen stärkerer Krümmung mit hoher Feldstärke. Die herrausragenden Phasen höherer Feldverdampfungsfeldstärke werden deshalb mit hellem Kontrast und die zurückweichenden Phasen geringerer Feldverdampfungsfeldstärke mit dunklem Kontrast im FIM abgebildet (Abb. 2.2). Diese deutliche Variation im lokalen Krümmungsradius führt neben den Kontrastunterschieden $\mathrm{zu}$ erheblicher Variation in der lokalen Vergrößerung und verhindert eine genaue Bestimmung der Teilchengrößen.

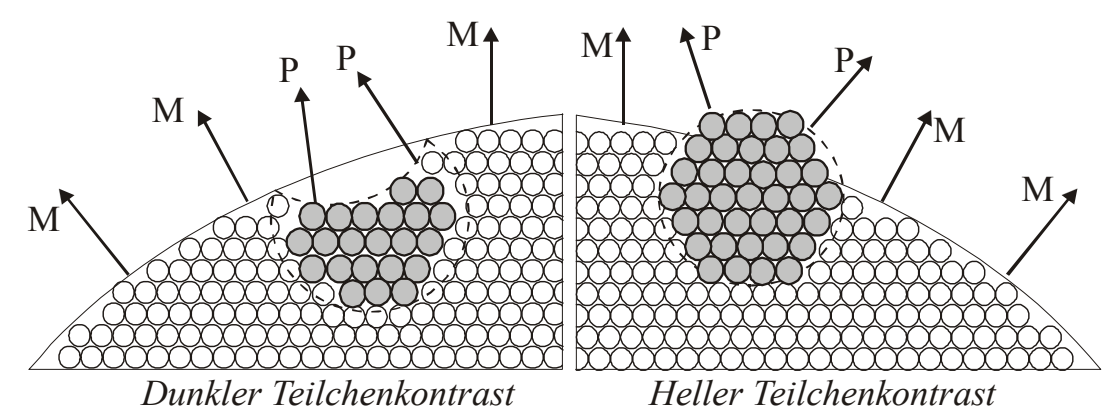

Abb. 2.2: $\quad$ FIM-Bild-Kontrast mit dunkel/hell abbildenden Teilchen anderer Phasen; Links: Ausscheidung hat geringere Feldverdampfungsfeldstärke als Matrix; Rechts: Ausscheidung hat höhere Feldverdampfungsfeldstärke als Matrix.. 


\subsubsection{Auswerteverfahren der Atomsondendaten}

Die Daten einer TAP-Messung werden gemäß der in Kap. 2.1.2 beschriebenen Punktprojektion zu einer Verteilung von Atomen rekonstruiert. Das Auswerteprogramm ermöglicht es, die so gewonnenen Informationen über Ort und Chemie der Atome optimal zu nutzen. In diesem Abschnitt werden die Methoden zur statistischen Auswertung des Datenmaterials vorgestellt.

\section{Leiterdiagramm}

Um Auskunft über die lokale Konzentration, z.B. an Grenzflächen, zu gewinnen, können sog. Leiterdiagramme angewandt werden. Die kumulative Anzahl der Ionen einer Komponente B wird hierbei gegen die Anzahl der Ionen aller Komponenten aufgetragen. Es entsteht ein Stufendiagramm, dessen Steigung direkt die Konzentration $\mathrm{c}_{\mathrm{B}}$ der Komponente $\mathrm{B}$ angibt. Um nachzuprüfen, ob signifikante Konzentrationsschwankungen um den Mittelwert auftreten, wird für Abschnitte mit unterschiedlichen Steigungen die doppelte Standardabweichung aus der Binomialstatistik als Vertrauensbereich angegeben:

$$
2 \sigma=2 \cdot \sqrt{\frac{\mathrm{c}_{\mathrm{B}}\left(1-\mathrm{c}_{\mathrm{B}}\right)}{\mathrm{N}}} .
$$

$\mathrm{N}$ ist hierbei die Anzahl der Ionen des Abschnitts und $\mathrm{c}_{\mathrm{B}}$ ist dessen Steigung. Nach der Binomialstatistik liegt die wahre Konzentration mit einer Wahrscheinlichkeit von 95,5\% innerhalb der $2 \sigma$-Grenzen. Auf diese Art läßt sich feststellen, ob eine signifikante Konzentrationsabweichung vom Mittelwert vorliegt, d.h. ob das untersuchte Gefüge homogen oder entmischt ist. Unterscheiden sich die Konzentrationswerte benachbarter Abschnitte außerhalb ihrer 2 $\sigma$-Grenzen, so geht man davon aus, daß es sich um eine signifikante Konzentrationsschwankung handelt. 


\section{Radiale Konzentrationsprofile}

Bei der Untersuchung von kugelförmigen Ausscheidungen einer zweiten Phase in der Matrix sind radiale Konzentrationsprofile besonders nützlich. Zunächst wird der Schwerpunkt einer Ausscheidung bestimmt. Anschließend werden Kugelschalen gleicher Schalendicke um das Zentrum der Ausscheidung gelegt. In jeder Kugelschale wird die lokale Konzentration bestimmt und gegen den Radius $r$ aufgetragen. Auf diese Art läßt sich auch ein Leiterdiagramm vom Ausscheidungszentrum bis zur Oberfläche eines Teilchens auftragen und somit direkt die lokale Konzentration an der Oberfläche der Ausscheidung ermitteln.

\section{Identifizierung von Ausscheidungen und Keimen}

Da bei konstantem Volumenbruchteil einer ausgeschiedenen Phase die Teilchenzahldichten bei kleinsten Ausscheidungen sehr hoch sind, wird es schwer, einzelne Ausscheidungen innerhalb des analysierten Volumens $\mathrm{zu}$ unterscheiden. Um die Frühstadien der Oxidkeimbildung zu charakterisieren, ist es außerdem wichtig, ein zuverlässiges Verfahren für die Unterscheidung von agglomerierten Atomen in signifikante sowie nicht signifikante Konzentrationsschwankungen zu finden. Die herkömmliche Auswertung über Isokonzentrationsflächen ist bei sehr kleinen Mengen an agglomerierten Atomen, im Folgenden als Cluster bezeichnet, nicht mehr anwendbar [FÄRBER00]. Im Rahmen dieser Arbeit wurde daher in Zusammenarbeit mit D. WoldeGiorgis [WoLDE03] und A. Heinrich [HEINRICH03] ein Algorithmus zur Identifizierung von oxidischen Clustern innerhalb der analysierten Volumina konzipiert und umgesetzt [KLUTHE03A]. Im Folgenden soll die Funktionsweise dieses Verfahrens vorgestellt werden:

Charakteristisch für einen Cluster aus B- Atomen in einer A- Matrix ist, daß der mittlere Abstand der B- Atome zueinander geringer ist, als in der homogenen Ausgangslegierung. Ziel des sog. Clustersuch- Algorithmus ist es daher, Bereiche innerhalb des Analysevolumens zu erfassen, in denen die Abstände der B- Atome untereinander kleiner sind, als ein vom Benutzer vorgegebener charakteristischer Abstand $r_{c}$. Der Algorithmus sucht dabei ausgehend von einem beliebigen B- Atom, die B- Atome, die 
sich innerhalb einer Kugel mit Radius $r_{c}$ um dieses Startatom befinden (Abb. 2.3, Schritt 1). Existieren solche Nachbaratome, so sucht er für diese wiederum alle Atome innerhalb $r_{c}$ (Schritt 2). Auf diese Art kann der Algorithmus einen Cluster rekursiv erschließen (Schritt 3). Eine weitere Bedingung für die Identifizierung des Clusters ist, daß dieser eine Mindestanzahl der Atome der Minoritätskomponente in einem zusammenhängenden Bereich enthält. Dies erscheint physikalisch sinnvoll, da man mit einem Cluster eine gewisse Anzahl von Atomen verbindet. Der Schwellwert $\mathrm{N}_{\mathrm{c}}$ für die Mindestanzahl der enthaltenen Atome ist somit ein weiterer Parameter, um einen statistisch signifikanten Cluster korrekt zu identifizieren.
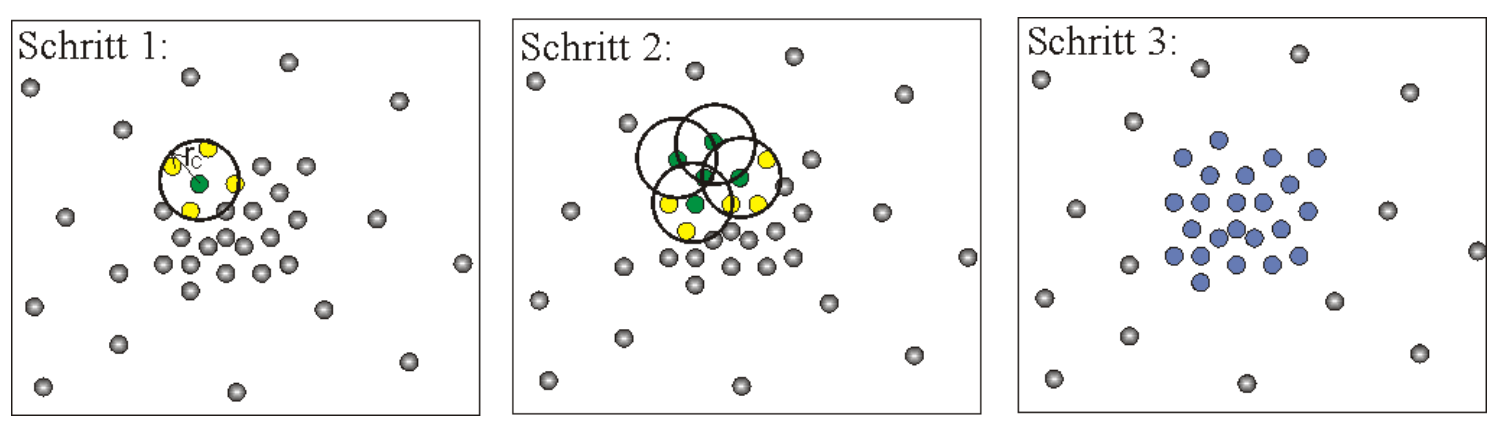

Abb. 2.3: Schematische Abbildung zur Darstellung der Funktionsweise des Clustersuch-Algorithmus; grün: beliebiges Startatom, gelb: Atom in Reichweite $r_{c}$ des Startatoms, blau: identifizierte Clusteratome.

Sind die zu untersuchenden Ausscheidungen durch einen Abbildungseffekt behaftet (siehe Kap. 2.1.3) wird die Identifizierung der analysierten Cluster erschwert. Als Folge eines lokalen Vergrößerungseffektes der Ausscheidungen, überlappen sich dann benachbarte Ausscheidungen innerhalb des Analysevolumens (Abb. 3.4a). Der oben beschriebene Clustersuch-Algorithmus definiert dann zwei sich überlappende Ausscheidungen als einen gemeinsamen Cluster. Um einzelne Ausscheidungen (aus BAtomen) dennoch korrekt $\mathrm{zu}$ identifizieren, besteht ein modifizierter Cluster-Suchalgorithmus aus 2 Schritten. Im ersten Schritt werden die B- Atome die sich außerhalb der A- freien Volumina befinden, aus dem Datensatz entfernt. Das sind solche BAtome mit einer signifikant höheren Volumendichte an Matrixatomen in ihrer Umgebung im Vergleich zu denen innerhalb der A- freien Bereiche. Diese Prozedur ist notwendig um gerade diejenigen B-Atome eines Teilchens zu entfernen die mit anderen Teilchen überlappen (siehe Abb. 3.4b). Im zweiten Schritt kann dann der oben 
beschriebene Clustersuch- Algorithmus angewendet, der nach charakteristischen Abständen der B- Atome sucht und so die Cluster innerhalb des extrahierten Datensatzes findet.

(a)

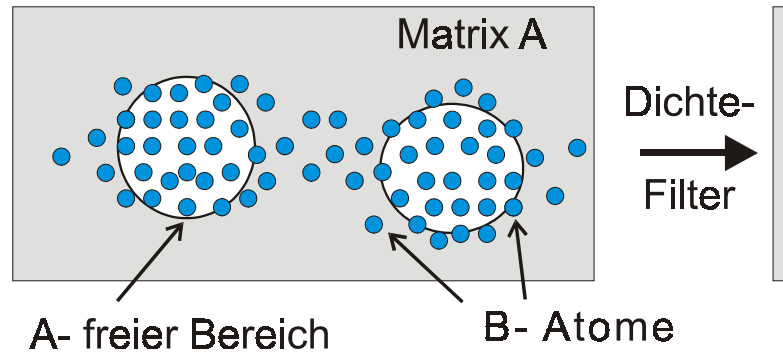

(b)

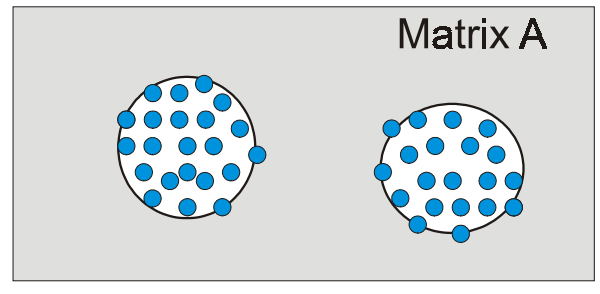

Abb. 2.4: 2D-Schematik zur Darstellung der Funktionsweise des DichtefilterAlgorithmus; (a) Zwei überlappende Ausscheidung aus B-Atomen innerhalb einer Matrix aus A-Atomen. (b) Nach Anwendung des Dichtefilters. 


\subsection{Neutronenstreuung}

Zur Untersuchung des Segregationsverhaltens von Wasserstoff und Deuterium innerhalb der innenoxidierten Proben wird in der vorliegenden Arbeit die Methode der Kleinwinkelneutronenstreuung (SANS) angewandt. Der Vorteil von SANS liegt in der, im Vergleich zu anderen Methoden hohen Sensitivität für Wasserstoff und in dem großen, analysierten Volumen von $0,1-1 \mathrm{~cm}^{3}$. Bei den zu untersuchenden Ag/MgOund $\mathrm{Ag} / \mathrm{Mn}_{3} \mathrm{O}_{4^{-}}$Proben wird damit typischerweise über $10^{16}$ bis $10^{17}$ Oxid- Teilchen gemittelt.

\subsubsection{Grundlagen der Streuung mit Neutronen}

Die Unterschiede der Neutronenstreuung zu anderen Streumethoden sind durch die Art der Wechselwirkung von Neutronen mit Materie begründet. Diese basiert auf den grundlegenden Eigenschaften des Neutrons:

$\begin{array}{llll}\text { Ladung } & \mathrm{Z}_{\mathrm{n}}=0 \\ \text { Masse } & \mathrm{m}_{\mathrm{n}}=1,675 \cdot 10^{-27} \mathrm{~kg} \\ \text { Spin } & \mathrm{S}_{\mathrm{n}}= & 1 / 2 \hbar \\ \text { Elektrisches Dipolmoment } & \mathrm{P}_{\mathrm{n}}=0 \\ \text { Magnetisches Dipolmoment } & \mu_{\mathrm{n}}=-1,913 \cdot \mu_{\text {Kern }} \\ \text { Halbwertszeit } & \tau_{1 / 2}=888 \mathrm{~s}\end{array}$

Nach dem von der Quantenmechanik beschriebenen Welle-Teilchen-Dualismus verhalten sich Neutronen wie Materiewellen. Neutronen kann somit nach de Broglie eine Wellenlänge zugeordnet werden:

$\lambda_{\mathrm{n}}=\frac{\mathrm{h}}{\mathrm{m}_{\mathrm{n}} \mathrm{v}_{\mathrm{n}}}=\frac{\mathrm{h}}{\sqrt{2 \mathrm{~m}_{\mathrm{n}} \mathrm{E}_{\mathrm{n}}}}$. 
Für kalte Neutronen, wie sie für die Neutronenstreuung verwendet werden, liegt diese im Bereich von Atomabständen in Festkörpern. Die Wechselwirkung mit Materie ist für Neutronen sehr viel geringer als für Röntgenquanten, da sie aufgrund ihrer fehlenden Ladung nur am Atomkern und nicht an dessen Hülle gestreut werden. Neutronen dringen daher sehr viel tiefer in Materie ein als Röntgenquanten.

Eine Folge der Streuung am Atomkern ist, daß verschiedene Isotope eines Elementes unterschiedlich stark streuen. Analog zum Strukturfaktor der Röntgenstreuung wird die Amplitude der Streuwelle als das Streuvermögen eines Atoms definiert. Man bezeichnet diese Größe als die Streulänge b, die vom Kernradius, der Kernstruktur, dem Kernspin und der Energie der Neutronen abhängt. Bei der Streuung an einem Kristall aus chemisch identischen Atomen sind zwei Anteile zu unterscheiden. Die kohärente Streuung wird durch eine mittlere Streulänge aller chemisch identischen Atome beschrieben:

$b_{\text {kohärent }}=\left\langle b_{i}\right\rangle$.

Die kohärent gestreuten Neutronen sind interferenzfähig und damit abhängig vom Streuvektor. Dies gilt nicht für die inkohärente Streuung, deren Streulänge $b_{i}^{\text {inkohärent }}$ als Wurzel der mittleren quadratischen Abweichung der Streulängen $b_{i}$ definiert ist:

$b_{\text {inkohärent }}=\sqrt{\left\langle b_{i}^{2}\right\rangle-\left\langle b_{i}\right\rangle^{2}}$.

Die Streulängen beziehen sich auf das Streuverhalten einzelner Atome. Als Materialeigenschaft wird zusätzlich die sog. Streulängendichte $\rho$ definiert, welche sich auf ein Volumen mehrerer Atome bezieht. Auf diese Art kann somit z.B. auch das Streuverhalten einer Phase aus chemisch unterschiedlichen Atomen beschrieben werden, nach:

$\rho=\frac{\sum_{i}^{N} b_{i}}{V_{N}}$. 
Die Streulängendichte $\rho$ kann dabei über eine beliebige Menge von N Atomen mit jeweiligen Volumen $\mathrm{V}_{\mathrm{N}}$ definiert werden. Die gewählte Menge darf allerdings innerhalb der Phase keine Inhomogenität in b zeigen, da diese Fluktuation einen zusätzlichen Streubeitrag verursachen würde. Die für diese Arbeit relevanten Streulängen sowie die Streulängendichten $\rho$ finden sich in Tabelle 2.1. Eine wichtige Besonderheit stellen die relativ großen Streulängen von $\mathrm{H}$ und $\mathrm{D}$ dar, die sogar unterschiedliche Vorzeichen aufweisen.

Tabelle 2.1: Kohärente und inkohärente Streulängen diverser Elemente sowie Streulängendichten relevanter Phasen.

\begin{tabular}{lllll}
\hline Element & $b_{\text {kohärent }}[\mathrm{fm}]$ & $b_{\text {inkohärent }}[\mathrm{fm}]$ & Phase & $\rho\left[10^{10} \mathrm{~cm}^{-2}\right]$ \\
\hline$H$ & $-3,74$ & 25,27 & $\mathrm{Ag}$ & 3,47 \\
$D$ & $+6,67$ & 4,04 & $\mathrm{MgO}$ & 5,98 \\
$O$ & $+5,80$ & 0 & $\mathrm{Mn}_{3} \mathrm{O}_{4}$ & 1,52 \\
$\mathrm{Mg}$ & $+5,38$ & 0,15 & & \\
$\mathrm{Mn}$ & $-3,73$ & 1,79 & & \\
$\mathrm{Ag}$ & $+5,92$ & -- & & \\
\hline
\end{tabular}

Die gestreute Intensität der Probe wird bei Streuexperimenten als Streuquerschnitt angegeben. Dabei bezeichnet der totale Streuquerschnitt $\sigma$ den Anteil der gestreuten Neutronen am gesamten Fluß einfallender Neutronen. Der differentielle Wirkungsquerschnitt $d \sigma$ pro Raumwinkelelement $\mathrm{d} \Omega$ und Volumen $\mathrm{V}$ wird dann als makroskopischer Streuquerschnitt $\mathrm{d} \Sigma / \mathrm{d} \Omega$ angegeben:

$\frac{\mathrm{d} \Sigma}{\mathrm{d} \Omega}=\frac{\text { Anzahl der nach } \mathrm{d} \Omega \text { gestreuter Neutronen pro Zeiteinheit }}{\text { gestreutes Volumen } \mathrm{V} \times \mathrm{Flu} ß \text { einallender Neutronen } \times \mathrm{d} \Omega}=\frac{1}{\mathrm{~V}} \frac{\mathrm{d} \sigma}{\mathrm{d} \Omega}$.

Den makroskopischen Streuquerschnitt erhält man aus der Interferenz von $\mathrm{N}$ Kugelwellen:

$$
\frac{\mathrm{d} \Sigma}{\mathrm{d} \Omega}=\frac{\mathrm{N}}{\mathrm{V}} \cdot\left|\iiint_{\text {Teilchen }} \Delta \rho \cdot \mathrm{e}^{\mathrm{i} \mathrm{Q} \cdot \overrightarrow{\mathrm{r}}} \mathrm{d}^{3} \overrightarrow{\mathrm{r}}\right|^{2}+\left.\frac{\mathrm{d} \Sigma}{\mathrm{d} \Omega}\right|_{\text {Inkohärent }}
$$


Dabei ist $\Delta \rho=\rho_{K}-\rho_{M}$ die Streulängendichtedifferenz des Teilchens zur Matrix und $\mathrm{d} \Sigma /\left.\mathrm{d} \Omega\right|_{\text {ink. }}$ der inkohärente Streuquerschnitt, welcher analog zu (2.9) definiert wird. Der inkohärente Streuquerschnitt ist in jedem Streuexperiment ein wesentlicher Bestandteil der Untergrundintensität, da er für alle Q- Vektoren identisch ist. Durch den hohen inkohärenten Streuquerschnitt des Wasserstoffs $\left(\sigma_{\text {ink }}^{\mathrm{H}}=4 \pi \cdot \mathrm{b}_{\text {ink }}^{\mathrm{H}}{ }^{2}=80,3\right.$ barn $)$ ist es möglich, aus der Zunahme der Untergrundintensität bei H- Beladung, nach Gl. (2.11) die H- Konzentration der Probe zu bestimmen:

$\mathrm{c}_{\mathrm{H}}=\left.\Delta \frac{\mathrm{d} \Sigma}{\mathrm{d} \Omega}\right|_{\mathrm{H}-\mathrm{UB}} \cdot \frac{4 \pi \cdot \mathrm{V}_{\mathrm{Ag}}}{\sigma_{\mathrm{inc}}^{\mathrm{H}}}$.

Hierbei ist $\Delta \mathrm{d} \Sigma /\left.\mathrm{d} \Omega\right|_{\mathrm{H}-\mathrm{UB}}$ die Zunahme der Untergrundintensität infolge der H-Beladung und $\mathrm{V}_{\mathrm{Ag}}$ das Atomvolumen des Ag.

\subsubsection{Prinzip der Neutronenkleinwinkelstreuung (SANS)}

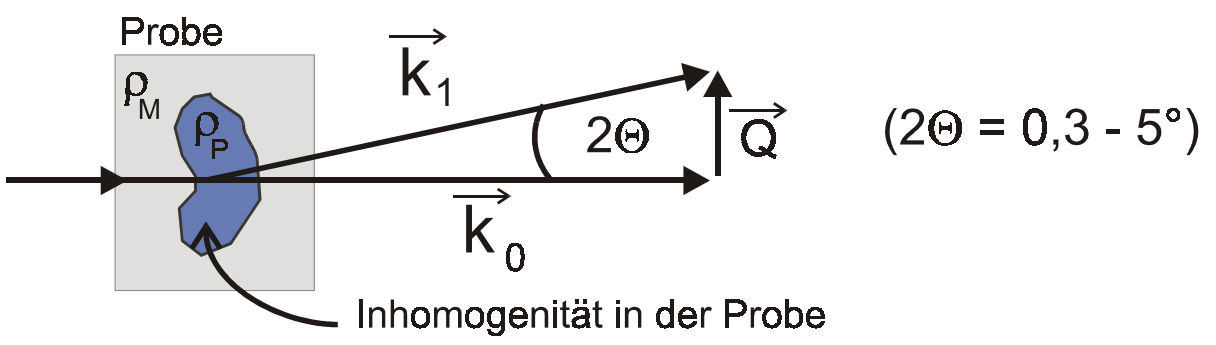

Abb. 2.5: Skizze zur Kleinwinkelstreuung. Streuung an einer Inhomogenität mit Streukontrast $\rho_{P}$ innerhalb einer Matrix mit Streukontrast $\rho_{M}$.

Bei kleinen Winkeln nahe dem Nullstrahl $\left(2 \Theta=0,3\right.$ bis $\left.5^{\circ}\right)$ wird das Streuverhalten durch räumliche Inhomogenitäten der Streulängendichte bestimmt. Objekte in Richtung des Streuvektors zeigen für bestimmte Q- Vektoren eine konstruktive Interferenz. Die Streuung von Objekten des Durchmessers d wird deshalb nur bei bestimmten QVektoren beobachtet:

$\mathrm{d}=\frac{2 \pi}{\mathrm{Q}}$ 
Folglich wird bei kleinen Q- Vektoren (d.h. Winkeln) nach (2.12) die Streuung größerer Inhomogenitäten und bei großen Q-Vektoren die Streuung kleinerer Inhomogenitäten beobachtet. Die gemessene Intensität hängt nicht nur von der Art der Matrix und der Teilchen ab, sondern auch von deren Volumenkonzentration, Form und Größe.

Die SANS- Untersuchungen dieser Arbeit wurden im Forschungszentrum Jülich an den Instrumenten KWS1 und KWS2 und im National Institute of Standards and Technology in Gaithersburgh M.D. (USA) am NG7-SANS- Instrument durchgeführt. Die Meßinstrumente sind im Wesentlichen, wie in Abb. 2.6 aufgebaut.

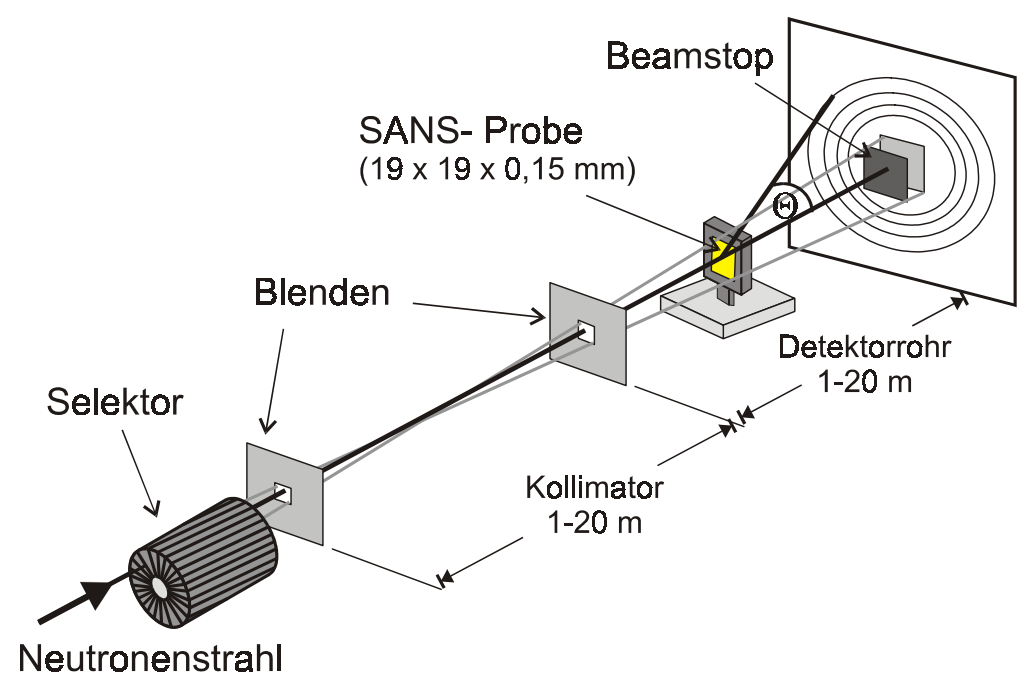

Abb. 2.6: Schematische Anordnung des SANS- Experimentes.

Thermische Neutronen werden in einem Moderator aus den heißen Neutronen des Reaktors produziert. Die Temperatur des Moderators liegt in der Größenordnung 50 bis $80^{\circ} \mathrm{C}$. Kalte Neutronen werden aus den thermischen Neutronen in einer sog. Kalten Quelle erzeugt und dann über Totalreflexion an Ni-58 beschichteten Glasleitern zu einem Wellenlängen- Selektor weitergeleitet. Dieser kann nur von Neutronen einer bestimmten Geschwindigkeit und somit auch Wellenlänge passiert werden. Der Neutronenstrahl wird anschließend durch eine Reihe von Blenden kollimiert, wobei Ein- und Austrittsblenden variabel sind, und trifft dann auf die Probe. Die gestreuten Neutronen werden auf einem 2D- Detektor registriert. Das Optimum von Auflösung und Intensität wird gerade dann erreicht, wenn der Kollimatorabstand gleich dem Abstand der Probe zum Detektor ist. Der Durchmesser des Neutronenstrahls im Probenort liegt für diese Arbeit bei $2 \mathrm{~cm}$. Die Dicke der durchstrahlten Proben liegt 
typischerweise im Zehntel mm-Bereich. Dies führt dazu, daß Untersuchungen mit kalten Neutronen grundsätzlich über das Probenvolumen gemittelte Aussagen erbringen. Je nach Querschnitt des auf die Probe einfallenden Neutronenstrahls beträgt das erfaßte Probenvolumen ca. $0,5-1 \mathrm{~cm}^{3}$.

\subsubsection{Streuung an kugelsymmetrischen Teilchen}

Die makroskopischen Streuquerschnitte einfacher Objekte weisen typische Streukurven auf. Allgemeine Darstellungen werden von Kostorz [KosTORZ93] und Glatter und Kratky [GLATTER83] gegeben. Im Folgenden werden die für diese Arbeit relevanten Guinier- und Porod- Näherungen vorgestellt. Beide beschreiben die Streuung kugelsymmetrischer Teilchen in homogener Matrix, die Guinierstreuung bei kleinen QVektoren mit exponentiellen Verhalten und das Porodverhalten bei etwas größeren QVektoren mit einer $\mathrm{Q}^{-4}$-Abhängigkeit der gestreuten Intensität.

\section{Guinierverhalten}

Die Streuung von Kugeln des Radius R, mit einer Streulängendichtedifferenz $\Delta \rho_{\mathrm{KM}}=$ $\rho_{K}-\rho_{M}$ zur homogenen Matrix und einer Volumendichte $N$ kann durch Ausführung des Integrals von Gl. (2.10) mit Hilfe des Kugelformfaktors (siehe Kap. 6.1) berechnet werden:

$$
\frac{\mathrm{d} \Sigma}{\mathrm{d} \Omega}=\mathrm{N} \cdot\left(\frac{4 \pi \cdot \Delta \rho_{\mathrm{KM}}}{\mathrm{Q}}\right)^{2} \cdot\left|\frac{\sin \mathrm{QR}}{\mathrm{Q}^{2}}-\frac{\mathrm{R} \cdot \cos \mathrm{QR}}{\mathrm{Q}}\right|^{2} .
$$

Für QR $\rightarrow 0$ entspricht das Betragsquadrat in der Guinier- Näherung [GUINIER63] einer Exponentialfunktion:

$$
\frac{\mathrm{d} \Sigma}{\mathrm{d} \Omega}=\frac{4}{3} \pi \cdot \mathrm{R}^{3} \cdot v \cdot \Delta \rho_{\mathrm{KM}}^{2} \cdot \exp \left(-\frac{(\mathrm{QR})^{2}}{5}\right)
$$


$v$ ist dabei der Volumenbruchteil der Kugeln. Die Guinier- Näherung gilt, allgemeiner formuliert, für beliebige Teilchenformen und ist über alle Orientierungen gemittelt:

$$
\frac{\mathrm{d} \Sigma}{\mathrm{d} \Omega}=\mathrm{N} \cdot \mathrm{V}^{2} \cdot \Delta \rho_{\mathrm{KM}}^{2} \cdot \exp \left(-\frac{\mathrm{Q}^{2}\left\langle\mathrm{R}_{\mathrm{G}}^{2}\right\rangle}{3}\right) \text {. }
$$

Dabei bezeichnet $\left\langle\mathrm{R}_{\mathrm{G}}{ }^{2}\right\rangle$ den Gyrationsradius des Teilchens, analog dem Trägheitsmoment. Für kugelförmige Teilchen mit Radius $R$ gilt gerade: $R^{2}=5 / 3 \cdot R_{G}{ }^{2}$. Die Guinier- Gleichung (2.15) beschreibt das Streuverhalten für $\mathrm{Q} \cdot \mathrm{R}_{\mathrm{G}}<1$.

\section{Porodverhalten}

Betrachtet man Gleichung (2.13) für den Fall $Q \cdot R_{G}>1$, so kann man das Betragsquadrat durch den Wert seines Maximums von eins annähern [GLATTER83]. Faßt man zusätzlich $\mathrm{N} \cdot 4 \pi \mathrm{R}^{2}$ zur Gesamtoberfläche $\mathrm{S}$ pro Einheitsvolumen zusammen, so erhält man das Porod- Gesetz:

$$
\frac{\mathrm{d} \Sigma}{\mathrm{d} \Omega}=\frac{4 \pi \cdot \mathrm{S} \cdot \Delta \rho_{\mathrm{KM}}^{2}}{\mathrm{Q}^{4}} .
$$

Das Porod- Gesetz gilt allgemein für Teilchen mit scharfen Grenzflächen und homogener Dichte. Insbesondere muß Q größer als der Reziprokwert der kürzesten Teilchendimension sein, damit $\mathrm{Q} \cdot \mathrm{R}_{\mathrm{G}}>>1$ erfüllt ist. Umgekehrt bedeutet dies, daß die Teilchendimension mindestens dem Reziprokwert des kleinsten Streuvektors entspricht, bei dem das Porodverhalten beobachtet wurde.

Abb. 2.7 zeigt eine Berechnung des zu erwartenden Streuverhaltens von MgO- Teilchen in Ag. Links im Graphen ist der Guinierbereich, rechts der Porodbereich zu erkennen. Die Modulationen im Porodbereich stammen aus dem Formfaktor für Streuung an kugelsymmetrischen Teilchen. Die Maxima dieser Modulationen fallen mit $\mathrm{Q}^{-4} \mathrm{ab}$ und ihre Lage ist durch den Teilchenradius R bestimmt. Für das n-te Maximum $\mathrm{Q}_{\operatorname{Max}}{ }^{\mathrm{n}}$ gilt:

$$
\mathrm{R} \cdot \mathrm{Q}_{\mathrm{Max}}^{\mathrm{n}}=\mathrm{n} \cdot \pi \quad, \text { mit } \mathrm{n}=1,2,3, \ldots
$$


Die schwarze Kurve gilt nur für eine exakt monodisperse Teilchengrößen- Verteilung. Mit zunehmender Verteilungsbreite gehen die Modulationen in einer Gerade mit $\mathrm{Q}^{-4}$ Verhalten über (vgl. Abb. 2.7).

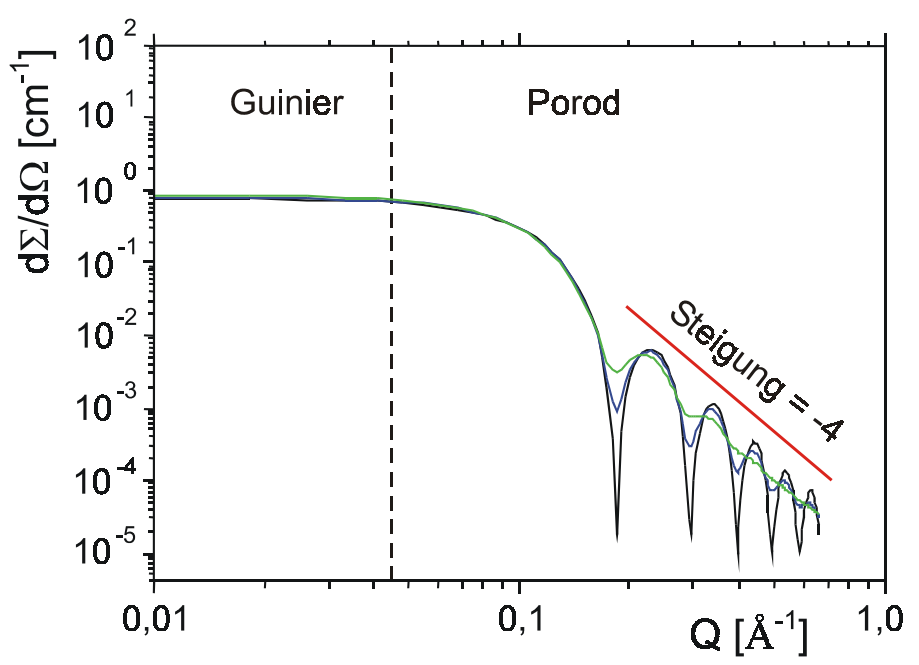

Abb. 2.7: Berechnete Streukurven für MgO- Teilchen in Ag unterschiedlicher Verteilungsbreite um einen mittleren Teilchenradius von $R=3 \mathrm{~nm}$; schwarz: $0 \%$, blau: $5 \%$, grün: $15 \%$ Polydispersität.

\subsubsection{Streuverhalten einer segregierten Schale H, D}

Im Rahmen dieser Arbeit wird die Segregation von Wasserstoff an M/O- Teilchengrenzflächen mit Hilfe von SANS untersucht. Da sowohl MgO-, als auch $\mathrm{Mn}_{3} \mathrm{O}_{4^{-}}$Ausscheidungen nahezu kugelförmig sind, sollte sich ein an diesen Teilchen segregierter Wasserstoff in guter Näherung als dünne Schale um ein kugelförmiges Oxidteilchen beschreiben lassen (siehe Abb. 2.8 und [KIRCHHEIM03,

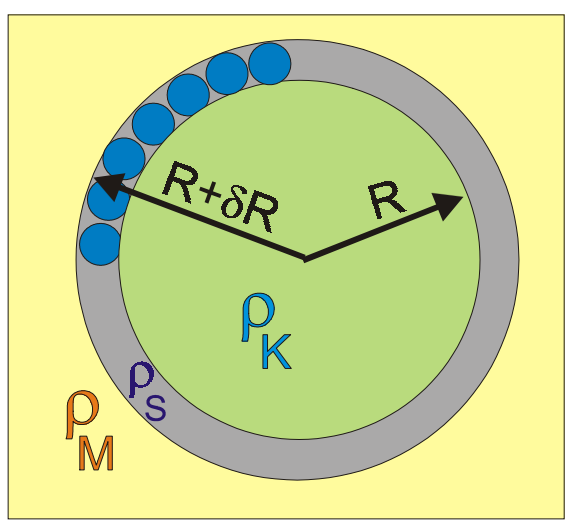

Abb. 2.8: Kugel-Schale Modell. KLUTHE03B]). Dieser Schale kann dann eine eigene Streulängendichte $\rho_{\mathrm{S}}$ zugeordnet werden. Segregiert der Wasserstoff an den Teilchengrenzflächen, ohne daß sich die Dichte in seiner Umgebung ändert, wird der Streukontrast $\Delta \rho_{\mathrm{S}}$ nach der Definition der Streulängendichte zu: 
$\Delta \rho_{\mathrm{S}}=\frac{\mathrm{N}_{\mathrm{H}} \cdot \mathrm{b}_{\mathrm{H}}}{\mathrm{V}}=\frac{\mathrm{N}_{\mathrm{H}} \cdot \mathrm{b}_{\mathrm{H}}}{\mathrm{A} \cdot \delta \mathrm{R}}=\frac{\Theta_{\mathrm{H}} \cdot \mathrm{b}_{\mathrm{H}}}{\delta \mathrm{R}}$.

$\mathrm{N}_{\mathrm{H}}$ ist die Anzahl segregierter H-Atome im Volumen V, mit kohärenter Streulänge $\mathrm{b}_{\mathrm{H}}$. $\Theta_{\mathrm{H}}$ ist hierbei die Belegungsdichte der Grenzflächen mit Wasserstoff. Berücksichtigt man, daß der Wasserstoff durch seine Segregation die Matrixdichte in der Nähe der Grenzfläche verändert, muß ein weiterer Term eingeführt werden, welcher die Menge der verdrängten Ag- Atome mit einbezieht:

$$
\Delta \rho_{\mathrm{S}}=\frac{\Theta_{\mathrm{H}} \cdot \mathrm{b}_{\mathrm{H}}-\Delta \Theta_{\mathrm{Ag}} \cdot \mathrm{b}_{\mathrm{Ag}}}{\delta \mathrm{R}} .
$$

$\Delta \Theta_{\mathrm{Ag}}$ ist hierbei die Änderung der Ag- Dichte mit $\mathrm{b}_{\mathrm{Ag}}$ als kohärente Streulänge des Silbers. Findet also eine Änderung der Ag- Dichte bei H- Segregation statt, wird der Kontrast um den Betrag des zweiten Terms vermindert. Aufgrund des negativen Vorzeichens der kohärenten Streulänge von Wasserstoff $\left(b_{H}=-3,74 \mathrm{fm}, b_{D}=+6,67 \mathrm{fm}\right)$ nimmt der Kontrast von Wasserstoff vom Betrag her zu, wohingegen der Kontrast des Deuteriums abnimmt. Die stärkere kohärente Streuung von Deuterium im Vergleich zu Wasserstoff wird somit bei einer Änderung der Ag- Dichte durch H/D- Segregation in einem gewissen Maße kompensiert.

Eine Herleitung aus Gl. (2.10) für den Spezialfall des makroskopischen Streuquerschnitts $\mathrm{d} \Sigma /\left.\mathrm{d} \Omega\right|_{\text {Schale }} ^{0}$ einer segregierten Schale um ein kugelförmiges Teilchen ist im Appendix in Kap. 6.1 angeführt. Für den Guinier- Bereich erhält man folgende Näherung für $\mathrm{d} \Sigma /\left.\mathrm{d} \Omega\right|_{\text {Schale }} ^{0}$ :

$$
\left.\frac{\mathrm{d} \Sigma}{\mathrm{d} \Omega}\right|_{\text {Schale }} ^{0}=\frac{\left.\frac{\mathrm{d} \Sigma}{\mathrm{d} \Omega}\right|_{\mathrm{K}+\mathrm{S}}-\left.\frac{\mathrm{d} \Sigma}{\mathrm{d} \Omega}\right|_{\mathrm{K}}}{\left.\frac{\mathrm{d} \Sigma}{\mathrm{d} \Omega}\right|_{\mathrm{K}}}=\frac{6 \Delta \rho_{\mathrm{S}} \delta \mathrm{R}}{\Delta \rho_{\mathrm{KM}} \mathrm{R}} \cdot \exp \left(-\frac{\mathrm{Q}^{2} \mathrm{R}^{2}}{15}\right) .
$$


Hierbei ist R der Teilchenradius und $\delta \mathrm{R}$ die Dicke der segregierten Schale. $\Delta \rho_{\mathrm{KM}}, \Delta \rho_{\mathrm{S}}$ sind die Differenzen der Streulängendichte von Teilchen zu Matrix und segregierter Schicht zu Matrix (siehe Abb. 2.8). In einem Streuexperiment entspricht $d \Sigma /\left.d \Omega\right|_{K}$ der gestreuten Intensität der unbeladenen Probe und $\mathrm{d} \Sigma /\left.\mathrm{d} \Omega\right|_{\mathrm{K}+\mathrm{S}}$ der gestreuten Intensität der mit $\mathrm{H}$ (bzw. D) beladenen Probe. $\mathrm{d} \Sigma /\left.\mathrm{d} \Omega\right|_{\mathrm{S}} ^{0}$ wird im Folgenden als normierter Nettostreuquerschnitt der Schale bezeichnet.

Aus einer modifizierten Guinier- Auftragung $\ln \mathrm{d} \Sigma /\left.\mathrm{d} \Omega\right|_{\mathrm{S}} ^{0}$ gegen $(\mathrm{QR})^{2}$ läßt sich nach Gleichung (2.20) das Produkt $\Delta \rho_{\mathrm{S}} \cdot \delta \mathrm{R}$ aus dem Achsenabschnitt direkt ermitteln. Dieses Produkt $\Delta \rho_{S} \cdot \delta \mathrm{R}$ aus Streukontrast der Schale und Schalendicke wird im Folgenden als Schalenkontrast bezeichnet. Da man nicht von einer reinen Segregation, d.h. einer Segregation ohne Änderung der Ag- Dichte, ausgehen kann, läßt sich aus dem Schalenkontrast nicht direkt auf die H- Belegungsdichte der Schale schließen. Aus Gl. (2.19) ist erkennbar, daß die Dichteänderung $\Delta \Theta_{\mathrm{Ag}}$ des $\mathrm{Ag}$ einen weiteren unbekannten Parameter darstellt. Um $\Theta_{\mathrm{H}}$ dennoch exakt zu bestimmen, kann man sich der Kontrastvariation von $\mathrm{H}$ und $\mathrm{D}$ bedienen. Da Wasserstoff und Deuterium chemisch identisch sind, sollten deren Belegungsdichten ebenfalls gleich sein, also $\Theta_{H}=\Theta_{D}$. Zieht man die normierten Nettostreuquerschnitte für Wasserstoff und Deuterium unter Verwendung der Gleichungen (2.19) und (2.20) voneinander ab, erhält man:

$$
\left.\frac{\mathrm{d} \Sigma}{\mathrm{d} \Omega}\right|_{\mathrm{D}-\mathrm{H}} ^{0}=\left.\frac{\mathrm{d} \Sigma}{\mathrm{d} \Omega}\right|_{\mathrm{D}} ^{0}-\left.\frac{\mathrm{d} \Sigma}{\mathrm{d} \Omega}\right|_{\mathrm{H}} ^{0}=\frac{6 \Theta_{\mathrm{H}}\left(\mathrm{b}_{\mathrm{D}}-\mathrm{b}_{\mathrm{H}}\right)}{\Delta \rho_{\mathrm{KM}} \mathrm{R}} \exp \left(-\frac{(\mathrm{QR})^{2}}{15}\right)
$$

Da die Teilchengröße R schon aus den unbeladenen Proben bestimmt werden kann, ist in (2.21) die H- Belegungsdichte $\Theta_{\mathrm{H}}$ der einzige unbekannte Parameter. Dieser läßt sich dann aus einer Auftragung von $\ln \mathrm{d} \Sigma /\left.\mathrm{d} \Omega\right|_{\mathrm{S}} ^{0}$ gegen $(\mathrm{QR})^{2}$ aus dem Achsenabschnitt bestimmen. Mit $\Theta_{\mathrm{H}}$, sowie den mit Gleichung (2.20) bestimmten Schalenkontrasten für $\mathrm{H}$ oder D kann nach (2.19) schließlich auch eine etwaige Ag- Dichteänderung $\Delta \Theta_{\mathrm{Ag}}$ berechnet werden. 


\subsection{Probenpräparation}

\subsubsection{Präparation der innenoxidierten Proben für TAP- Analysen}

Um die Frühstadien der Oxidbildung mit TAP zu charakterisieren, muß die Anzahldichte der gebildeten Ausscheidungen möglichst klein sein. Nur so sind die einzelnen Teilchen soweit voneinander entfernt, daß eine eindeutige Separation und Charakterisierung der Oxidausscheidungen möglich ist. Da mit geringerer $\mathrm{Mg}$ - Einwaage die Anzahldichten kleiner werden, die Teilchengrößen hingegen nahezu gleich bleiben [CHARRIN00], wurden für die TAP- Analysen Ag- Legierungen mit 0,4 At.\% Mg bzw. 0,4 At.\% Mn im Lichtbogenofen hergestellt ${ }^{4}$. Die aufgeschmolzenen Kügelchen wurden dann bei $650{ }^{\circ} \mathrm{C}$ für 4 Wochen unter Argon- Schutzgas homogenisiert und daraufhin zu Drähten von $200 \mu \mathrm{m}$ Durchmesser gezogen. Anschließend folgte eine Rekristallisationsbehandlung. Diese war notwendig, um den Einfluß der Korngrenzen bei der Inneren Oxidation der Proben zu minimieren [KLUTHE03A]. Nach einer Glühung bei $650{ }^{\circ} \mathrm{C}$ für $24 \mathrm{~h}$ unter Argon- Atmosphäre wurde eine mittlere Korngröße von 80 $\mu \mathrm{m}$ für die Ag-0,4At.\%Mg- und $150 \mu \mathrm{m}$ für die Ag-0,4At.\%Mn- Proben erzielt. Um eine homogene O- Permeation zu gewährleisten, wurden die Proben direkt vor der Inneren Oxidation in einer Lösung aus $\mathrm{NH}_{3}, \mathrm{H}_{2} \mathrm{O}_{2}$ und $\mathrm{H}_{2} \mathrm{O}$ (Verhältnis 15:3:5) für wenige Sekunden angeätzt.

Die Ag-0,4At.\%Mg- Legierung wurde bei $\mathrm{T}_{\mathrm{IO}}=750{ }^{\circ} \mathrm{C}$ für verschiedene Zeiten $\mathrm{t}_{\mathrm{IO}}=10$ min bis 182 d an Luft innenoxidiert. Für die Ag-0,4At.\%Mn- Legierung wurde die Innere Oxidation bei $\mathrm{T}_{\mathrm{IO}}=650^{\circ} \mathrm{C}$ für $\mathrm{t}_{\mathrm{IO}}=10 \mathrm{~min}$ bis $21 \mathrm{~d}$ durchgeführt. Für die TAPAnalyse wurden dann für beide Legierungen die jeweiligen Drähte bei 8 V DC elektrolytisch zu FIM- Spitzen gedünnt. Als Elektrolyt diente eine Mischung aus Perchlorsäure und Essigsäure (1:10) und als Kathode wurde eine Platin- Elektrode benutzt. Die Qualität der hergestellten TAP- Proben konnte anschließend im Elektronenmikroskop überprüft werden (Abb. 2.9).

\footnotetext{
${ }^{4}$ Reinheit der Ausgangsstoffe: Ag 99,999 \%, Mg und Mn: 99,95\%.
} 


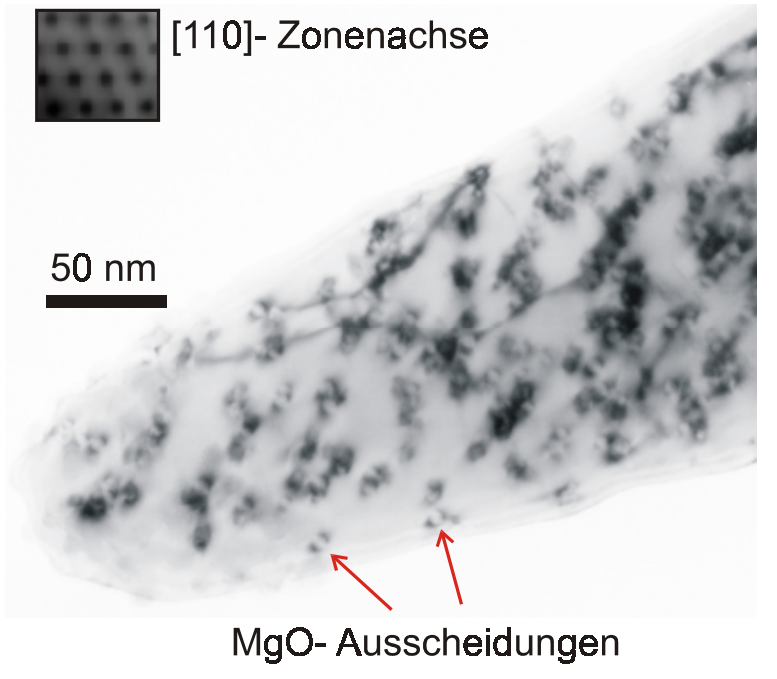

Abb. 2.9: TEM-Aufnahme einer innenoxidierten AgMg- Spitze. Die $\mathrm{MgO}$ Teilchen sind durch einen Kaffeebohnenkontrast sichtbar.

\subsubsection{Präparation der SANS- Proben}

Die für die SANS- Analysen verwendete Ag-1At.\%Mg- Legierung wurde von der Fa. Vacuumschmelze Hanau GmbH freundlicherweise zur Verfügung gestellt. Die Ag1At.\%Mn- Legierung konnte im Lichtbogenofen analog zu den Proben in Kap. 2.3.1 hergestellt werden. Diese Legierungen wurden dann zu Folien von $150 \mu \mathrm{m}$ Dicke gewalzt und bei $650{ }^{\circ} \mathrm{C}$ für $24 \mathrm{~h}$ im UHV rekristallisiert. Die Korngröße der $\mathrm{Ag}$ 1At.\%Mg- Legierung betrug nach der Rekristallisationsbehandlung $100 \mu \mathrm{m}$, die der Ag1At.\%Mn- Legierung $200 \mu \mathrm{m}$. Die Innere Oxidation wurde bei Temperaturen von 650 bis $850{ }^{\circ} \mathrm{C}$ für 1 bis 27 Tage an Luft durchgeführt. Anschließend wurde die Oberfläche der SANS- Proben elektrolytisch analog zu Kap. 2.3.1 bei 2-3 V DC poliert. Eine Mikrosondenanalyse (Abb. 2.10) der so hergestellten Ag-1At.\%Mg- Proben bestätigt eine homogene $\mathrm{MgO}$ - Konzentration von $\mathrm{c}_{\mathrm{Mg}}=(0,81 \pm 0,02)$ At.\% innerhalb eines AgKorns. Die Ag-1At.\%Mn- Proben wiesen eine mittlere Konzentration von $\mathrm{c}_{\mathrm{Mn}}=(0,98 \pm$ 0,02) At.\% innerhalb der Ag- Körner ${ }^{5}$ auf.

\footnotetext{
${ }^{5}$ Diese Konzentrationen entsprechen 0,9 Vol.\% der MgO-, sowie 1,5 Vol.\% der $\mathrm{Mn}_{3} \mathrm{O}_{4}$ - Phase innerhalb der Ag- Körner der jeweiligen Proben.
} 

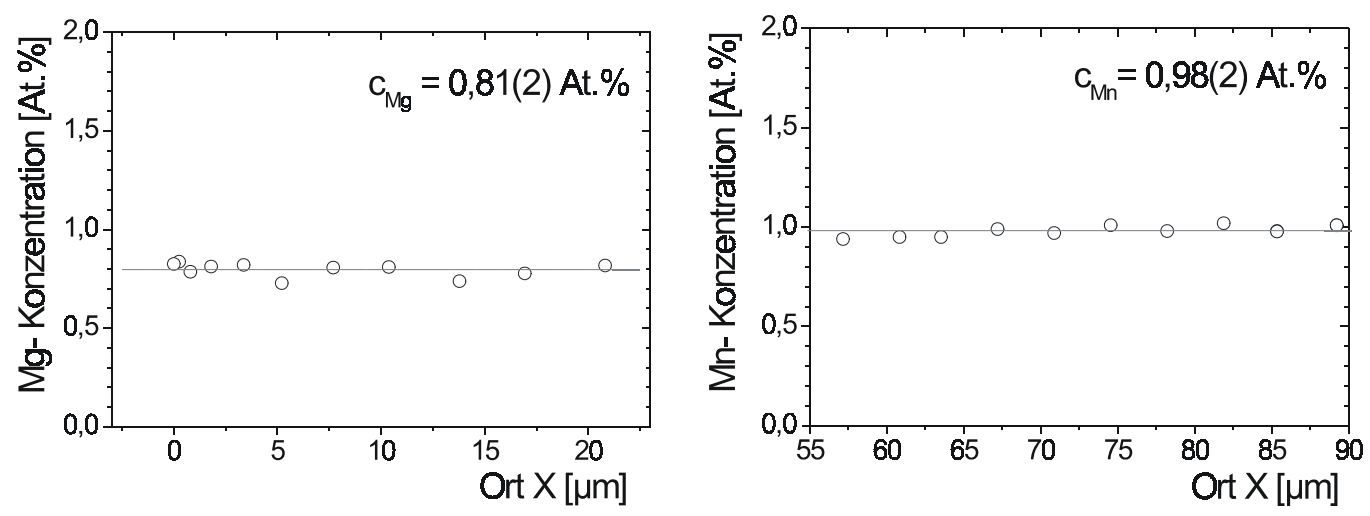

Abb. 2.10: Mikrosondenanalysen der innenoxidierten SANS-Proben.

\subsubsection{H,D- Beladung der Proben}

Um die Segregation des $\mathrm{H}$ an den M/O- Grenzfläche zu studieren, wurden die innenoxidierten Proben einer H- Beladung unterzogen (siehe Kap. 1.2.1). Damit gewährleistet ist, daß die innenoxidierten Proben vollständig beladen werden, muß die erforderliche Beladungstemperatur und -zeit abgeschätzt werden. Geht man davon aus, daß der Wasserstoff an den Teilchengrenzflächen segregiert, so gilt für dessen Eindiffusion in die Probe ein parabolisches Wachstumsgesetz (analog zur Bewegung der Oxidationsfront, siehe Kap. 1.1):

$\xi_{H}(t)=\sqrt{\frac{2 c_{H}^{0} \cdot D_{H} \cdot t}{c_{H}^{M / O}}}$.

$\xi_{\mathrm{H}}(\mathrm{t})$ ist hierbei der Ort der H- Diffusionsfront, $\mathrm{D}_{\mathrm{H}}$ der Diffusionskoeffizient und $\mathrm{c}_{\mathrm{H}}{ }^{0}$ die Löslichkeit von $\mathrm{H}$ in Ag. Nach Gegner beträgt die maximale $\mathrm{H}-$ Konzentration $\mathrm{c}_{\mathrm{H}}{ }^{\mathrm{M} / \mathrm{O}}$ einer innenoxidierten Ag-1At.\%Mg- Legierung etwa 0,1 At. \% [GEGNER95]. Auf Basis der Werte H- Löslichkeit und Diffusion in Ag aus [FrOMm76, OpARA97]

$\log \mathrm{c}_{\mathrm{H}}^{0}=\frac{1}{2} \log \mathrm{p}-1,66-\frac{3600}{\mathrm{~T}}, \mathrm{D}_{\mathrm{H}}(\mathrm{T})=2 \cdot 10^{-2} \cdot \exp \left(\frac{-7500}{\mathrm{RT}}\right)$, 
kann damit die Beladungszeit nach Gl. (2.24) für eine Diffusionsdistanz von $\xi_{\mathrm{H}}{ }^{\mathrm{Max}}=\mathrm{d} / 2$ $=75 \mu \mathrm{m}$ abgeschätzt werden.

$\mathrm{t}_{\mathrm{H}}=\frac{\mathrm{c}_{\mathrm{H}}^{\mathrm{M} / \mathrm{O}} \cdot \xi_{\mathrm{H}}^{\operatorname{Max}^{2}}}{2 \mathrm{c}_{\mathrm{H}}^{0} \cdot \mathrm{D}_{\mathrm{H}}}$.

Wie in Abb. $2.11 \mathrm{zu}$ erkennen, kann die H- Beladung nicht bei Raumtemperatur erfolgen, weil dies $\mathrm{zu}$ extrem langen Zeiten führen würde. Dies liegt an der sehr geringen Löslichkeit und Diffusion von $\mathrm{H}$ in Ag. Auch im Fall einer elektrolytischen Beladung wird diese Zeit nicht hinrei-

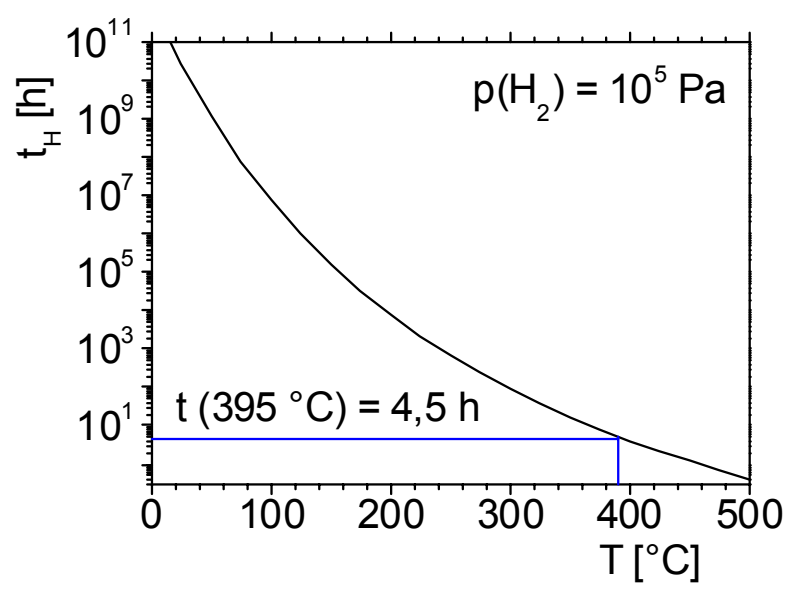
chend verkürzt, obwohl weitaus höhere $\mathrm{H}_{2}$ - Drücke erzielt werden

Abb. 2.11: H-Beladungsdauer nach Gl. (2.24). können. Die H- und D- Beladung der SANS- Proben wurde deshalb, basierend auf dieser Abschätzung, bei $395{ }^{\circ} \mathrm{C}$ für $8 \mathrm{~h}$ bei 1 bar $\mathrm{H}_{2}$ (bzw. $\mathrm{D}_{2}$ ) aus der Gasphase durchgeführt. Bei der gewählten Temperatur findet kein weiteres Wachstum der Oxidteilchen statt, auch sind keine weiteren Kornvergröberungsprozesse zu erwarten [SPRENGLER65], so daß zwar H (bzw. D) schneller aufgenommen wird, jedoch das Probengefüge unverändert bleibt. Die $\mathrm{H}$ - und D- Beladung der hergestellten $\mathrm{Fe} / \mathrm{MgO} / \mathrm{Ag}$ - und $\mathrm{Ag} / \mathrm{MgO} / \mathrm{Ag}$ Schichten (Appendix, Kap. 6.2) sowie der innenoxidierten TAP- Proben wurde bei 200 ${ }^{\circ} \mathrm{C}$ für 30 min durchgeführt. Aufgrund der kleineren Dimensionen ( $50 \mathrm{~nm}$ am Apex) konnte eine geringere Beladungstemperatur gewählt werden. 


\section{Ergebnisse}

Im Folgenden werden die Ergebnisse zur Bildungs- und Wachstumskinetik der Oxidausscheidungen sowie $\mathrm{zu}$ deren Grenzflächenzusammensetzung vorgestellt. Als repräsentative Modellsysteme wurden mittels Innerer Oxidation hergestellte MgOsowie $\mathrm{Mn}_{3} \mathrm{O}_{4}$ - Ausscheidungen in Ag- Matrix untersucht. Der Schwerpunkt lag hierbei auf dem System $\mathrm{Ag} / \mathrm{MgO}$, das wesentlich umfangreicher untersucht wurde als das System $\mathrm{Ag} / \mathrm{Mn}_{3} \mathrm{O}_{4}$. Da für die Untersuchung der $\mathrm{M} / \mathrm{O}-$ Grenzfläche ein gutes Verständnis der Bildungs- und Wachstumskinetik der Oxidausscheidungen von Vorteil ist, wurde zunächst die Kinetik der Inneren Oxidation der betreffenden Systeme mit Hilfe der tomographischen Atomsonde (TAP) studiert. Durch die Kombination von 3DAtomsondentomographie (TAP) und Neutronenkleinwinkelstreuung (SANS) konnte anschließend die systematische Untersuchung der M/O- Grenzfläche durchgeführt werden. Da der Wasserstoff als Sonde für die Charakterisierung der M/O- Grenzfläche benutzt werden kann (siehe Kap. 1.2), wurde im speziellen das Segregationsverhalten von Wasserstoff bzw. Deuterium untersucht.

\subsection{TAP- Ergebnisse des Systems Ag/MgO}

\subsubsection{Innere Oxidation der AgMg- Legierung}

Zur Analyse der zeitlichen Entwicklung von Morphologie und Größe der Oxidteilchen wurden Auslagerungsexperimente an zylinderförmigen Rohlingen der Zusammensetzung Ag-0,4At.\%Mg durchgeführt. Die Auslagerungsdauer bzw. Oxidationszeiten lagen dabei zwischen 10 Minuten und 182 Tagen bei einer Oxidationstemperatur von $750{ }^{\circ} \mathrm{C}$ (siehe auch Tabelle 3.1). Die Abb. 3.1a zeigt eine FIM- Abbildung einer für 7 Tage oxidierten Probe. Die MgO- Teilchen sind sehr leicht durch ihren hellen Kontrast im Vergleich zur dunkel abbildenden Ag- Matrix sichtbar. Für die quantitative Analyse wurden TAP- Untersuchungen durchgeführt, in denen die Oxidteilchen mit Hilfe des in Kap. 2.1.4 beschriebenen Cluster- Suchalgorithmus identifiziert wurden. Exemplarisch ist in Abb. 3.1b,c das Ergebnis dieses Verfahrens für eine 28 Tage lang oxidierte Probe 
zu sehen. Fast alle Mg- und O- Atome sind innerhalb von Ausscheidungen agglomeriert. Die Teilchen erscheinen etwas in die Breite gezogen zu sein. Dies ist eine Konsequenz eines lokalen Vergrößerungseffektes (siehe Kap. 2.1.3 und [KLUTHE02]). Bedingt durch diesen Meßeffekt wird bei der Bestimmung der Teilchengröße aus der Ausdehnung der zugehörigen Mg- u. O- Atome ein zu großer Wert für die Teilchengröße ermittelt.

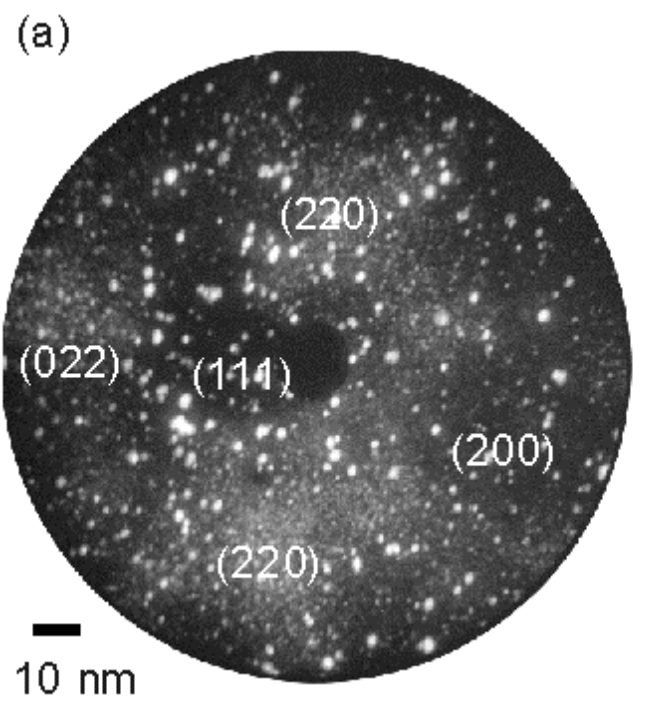

(b)

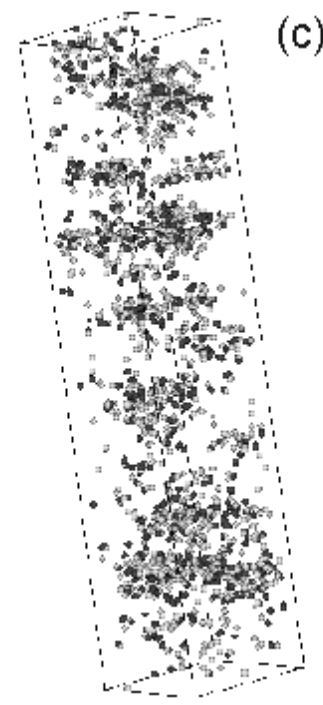

(c)

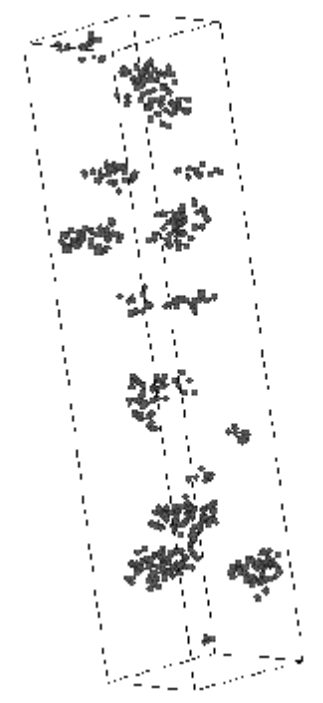

Abb. 3.1: (a) FIM-Abbildung einer für 7d innenoxidierten Probe. Die Pole niedriger Ordnung sind indiziert. Die MgO-Ausscheidungen sind durch ihren hellen Kontrast zur umgebenden Ag- Matrix sichtbar. (b) TAP-Analysevolumen einer für $28 d$ innen-

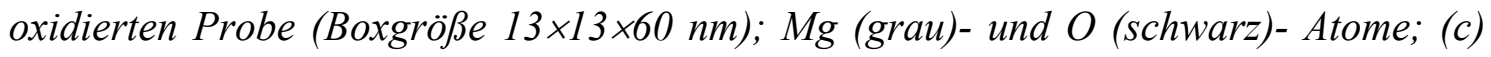
Verteilung der Oxidatome nach Verwendung des Cluster-Suchalgorithmus.

Die Feldverdampfung der Matrix- Atome wird hingegen nur unwesentlich von dem lokalen Vergrößerungseffekt der Teilchen beeinflußt [KLUTHE02]. Dies zeigt sich beispielsweise in Abb. 3.2 daran, daß bei einer Analyse nahe der (111)- Achse der Probe die Netzebenen innerhalb der Ag- Matrix aufgelöst werden. Aus diesem Grunde wurde das Ausmaß in x-, y- und z- Richtung des zugehörigen silberfreien Bereiches jeder Ausscheidung bestimmt. Anschließend wurde daraus die Teilchengröße als geometrisches Mittel dieser Werte bestimmt. 


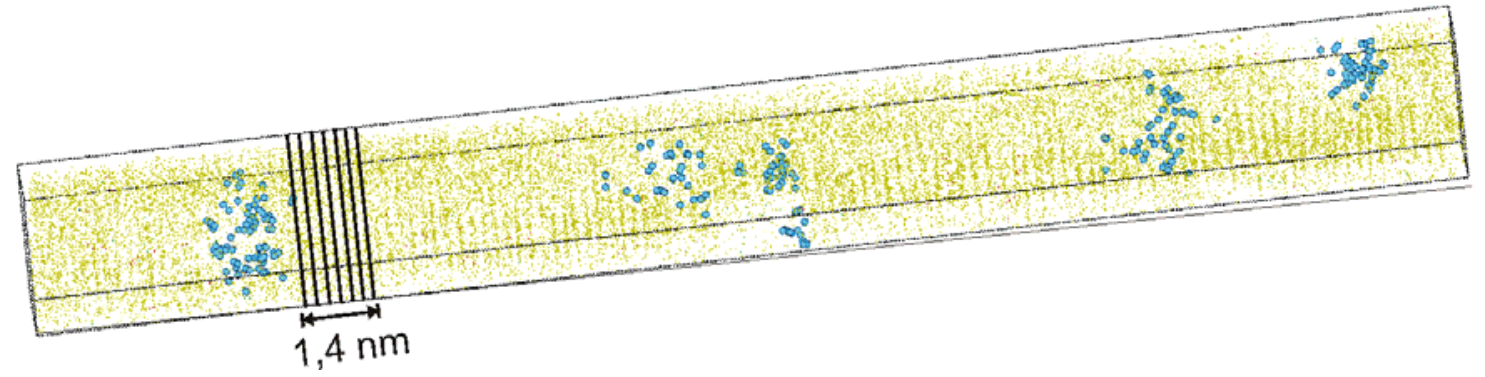

Abb. 3.2: Rekonstruiertes Meßvolumen einer für $t_{I O}=21$ Tage innenoxidierten Ag0,4At.\%Mg-Probe. \{111\}-Netzebenen innerhalb der Ag-Matrix (gelb) um mehrere $\mathrm{MgO}$ - Ausscheidungen (blau).

Abb. 3.3 zeigt die Ergebnisse der TAP- Untersuchungen der durchgeführten Auslagerungsserie. In dieser Abbildung wird eine Darstellung der Teilchen durch deren zugehörige silberfreie Bereiche bevorzugt, in der die Oxidteilchen als offensichtliche Lücken in der Matrix zu erkennen sind.
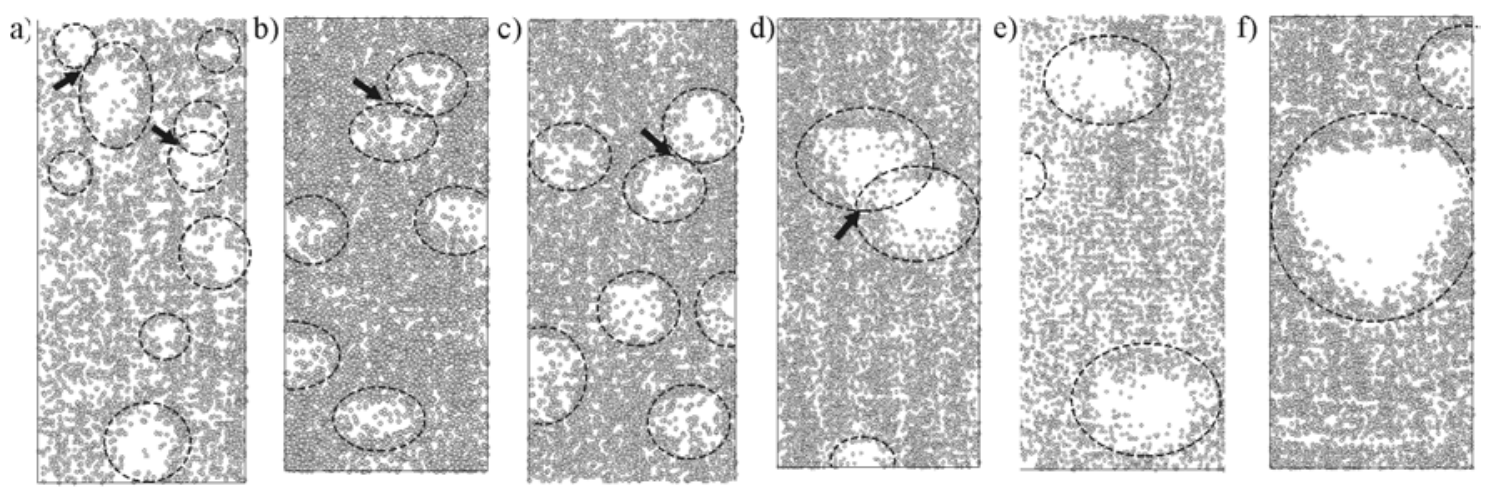

Abb. 3.3: Verteilung der Ag-Atome innerhalb rekonstruierter Analysevolumina von bei $750{ }^{\circ} \mathrm{C}$ für verschiedene Zeiten innenoxidierten Ag-0,4At.\%Mg-Proben; (a) $10 \mathrm{~min}$, (b) $1 \mathrm{~h}$, (c) $7 d$, (d) $12 d$, (e) $21 d$, (f) $28 d$. Die dargestellten Ausschnitte der Analysevolumina haben die Ausmaße: $9 \times 2 \times 20 \mathrm{~nm}$.

Die mittlere Größe der gebildeten Oxidteilchen nimmt mit steigender Oxidationszeit zu, wobei immer weniger Teilchen im Analysevolumen zu finden sind, die Anzahldichte also abnimmt. Die mittleren Teilchenradien und die entsprechenden Anzahldichten, wie sie mit Hilfe des Cluster- Suchalgorithmus gefunden wurden sind für die untersuchten Oxidationszeiten in Tabelle 3.1 aufgelistet. Typischerweise wurden für jeden Zustand etwa 100 Teilchen ausgezählt, um den Mittelwert der Teilchenradien hinreichend genau zu bestimmen. 
Tabelle 3.1: Aus TAP-Analysen bestimmte Werte von Radius $R$ und Anzahldichte $N_{V}$ der $\mathrm{MgO}$ - Ausscheidungen, sowie die jeweiligen O/Mg-Verhältnisse innerhalb der Proben für verschiedene Oxidationsdauern $t_{I O},\left(T_{I O}=750{ }^{\circ} \mathrm{C}\right)$. Es sind nur die statistischen Fehler der einzelnen Werte angegeben.

\begin{tabular}{l|cccccccccc}
\hline$t_{I O}$ & $10 \min$ & $35 \mathrm{~min}$ & $1 \mathrm{~h}$ & $6 \mathrm{~h}$ & $1 d$ & $7 d$ & $12 d$ & $21 d$ & $28 d$ & $182 d$ \\
\hline \multirow{2}{*}{$\mathrm{R}[\mathrm{nm}]$} & 0,55 & 0,88 & 0,91 & 0,94 & 1,00 & 1,41 & 1,64 & 1,70 & 1,99 & 3,10 \\
& $\pm 0,08$ & $\pm 0,07$ & $\pm 0,07$ & $\pm 0,04$ & $\pm 0,05$ & $\pm 0,06$ & $\pm 0,07$ & $\pm 0,06$ & $\pm 0,06$ & $\pm 0,09$ \\
\multirow{2}{*}{$\mathrm{N}_{\mathrm{V}}\left[10^{24} \mathrm{~m}^{-3}\right]$} & 2,00 & 1,85 & 1,80 & 1,78 & 1,40 & 0,60 & 0,32 & 0,29 & 0,25 & 0,05 \\
& $\pm 1,00$ & $\pm 0,37$ & $\pm 0,36$ & $\pm 0,36$ & $\pm 0,28$ & $\pm 0,12$ & $\pm 0,07$ & $\pm 0,06$ & $\pm 0,05$ & $\pm 0,01$ \\
$\mathrm{O} / \mathrm{Mg}$ & 0,82 & 0,83 & 0,89 & 0,85 & 0,80 & 0,73 & 0,79 & 0,80 & 0,77 & 0,82 \\
& $\pm 0,06$ & $\pm 0,13$ & $\pm 0,05$ & $\pm 0,06$ & $\pm 0,09$ & $\pm 0,11$ & $\pm 0,06$ & $\pm 0,10$ & $\pm 0,05$ & $\pm 0,06$ \\
\hline
\end{tabular}

Die bei $750{ }^{\circ} \mathrm{C}$ für nur 10 min oxidierte Probe weist eine sehr hohe Teilchenzahldichte auf. Der Vergleich der gemessenen Mg- und O- Verteilung mit der einer Binomialverteilung liefert einen Chi- Quadrat Wert $\chi^{2}$ Exp., der deutlich oberhalb des Vergleichswertes $\chi^{2}$ stat. liegt (Tabelle 3.2). Hierbei bezieht sich der Vergleichwert auf ein Signifikanzniveau von 95 \%. Folglich liegen sowohl Mg als auch O inhomogen verteilt innerhalb der Probe vor.

Tabelle 3.2: $\quad \chi^{2}$ - Test der Mg- und O-Verteilung innerhalb einer bei $750{ }^{\circ} \mathrm{C}$ für 10 min innenoxidierten Ag-0,4At.\%Mg-Probe.

\begin{tabular}{c|cc}
\hline Element & $\chi_{\text {Exp. }}^{2}$ & $\chi_{\text {Stat. }}^{2}$ \\
\hline $\mathrm{Mg}$ & 445 & 11 \\
\hline $\mathrm{O}$ & 429 & 11 \\
\hline
\end{tabular}

Anhand der Ag- freien Bereiche innerhalb der Analysevolumina konnte für diesen Zustandes ein mittlerer Teilchenradius von $\mathrm{R} \approx 0,6 \mathrm{~nm}$ bestimmt werden. Es ist mit den bisher entwickelten Methoden allerdings nicht möglich, Teilchen mit einem Radius von weniger als 0,4 $\mathrm{nm}$ innerhalb des Analysevolumens $\mathrm{zu}$ identifizieren. Für diesen Zustand liegt der tatsächliche Wert des mittleren Teilchenradius deshalb vermutlich 
unterhalb des ermittelten. Aus dem gleichen Grund ist anzunehmen, daß für diesen Zustand die Teilchenzahldichte höher ist als die aus den TAP- Analysen bestimmte.

Bei der Entwicklung der Teilchengröße fällt auf, daß sich schon nach einer Oxidationsdauer von 1 Stunde $\mathrm{MgO}$ - Ausscheidungen von etwa $\mathrm{R}=0,9 \mathrm{~nm}$ Radius gebildet haben. Bis $\mathrm{zu}$ einer Auslagerungszeit von $\mathrm{t}_{\mathrm{IO}}=24$ Stunden wird dann allerdings weder eine signifikante Größenzunahme der Ausscheidungen noch eine Abnahme in deren Anzahldichte Nv beobachtet. Erstaunlicherweise werden die Ausscheidungen für Zeiten länger als 24 Stunden wieder deutlich größer. Das Verhältnis aller Mg- und O- Atome der TAP- Analysen beträgt im Mittel über alle Oxidationszeiten $\mathrm{O} / \mathrm{Mg} \approx 0,8$ (Tabelle 3.1), abweichend von gravimetrischen Analysen mit $\mathrm{O} / \mathrm{Mg}>1$ [SEMEGA92]. Es ist somit davon auszugehen, daß im TAP nur etwa 75-80\% des Probensauerstoffs detektiert werden, da ein gewisser Teil des Probensauerstoffs zwischen den Hochspannungspulsen zusammen mit Wasserstoff des Restgases als HO- Komplex von der FIM- Spitze desorbiert [KLUTHE03A]. In diesem Fall ist der Sauerstoff für die Analyse verloren, da ihm keine Flugzeit zugeordnet werden kann.

Ferner wurde das Reifungsverhalten von Proben ohne Exzeß- O untersucht. Dazu wurden zwei Proben zunächst bei $750{ }^{\circ} \mathrm{C}$ für 35 min innenoxidiert. Eine dieser beiden Proben wurde dann im UHV bei $700{ }^{\circ} \mathrm{C}$ für $2 \mathrm{~h}$ geglüht. Durch die Glühung im UHV kann der überwiegende Teil des Exzeß- $\mathrm{O}$ aus dieser Probe entfernt werden. Anschließend wurde die UHV- geglühte Probe im Quarzglas unter 5.10 $\mathrm{Pa}$ Argon eingeschmolzen. Die zweite Probe wurde hingegen nicht behandelt. Beide Proben wurden dann im gleichen Muffelofen bei $750{ }^{\circ} \mathrm{C}$ für 7 Tage $(=168 \mathrm{~h})$ gealtert und anschließend einer TAP- Analyse unterzogen. Die aus den TAP- Analysen ermittelten Teilchenradien finden sich in Tabelle 3.3.

Tabelle 3.3: Bestimmte mittlere Teilchenradien von bei $750{ }^{\circ} \mathrm{C}$ für 7 Tage innenoxidierten Proben; (UHV) Probe ohne Exzeß-O, (O) Probe mit Exzeß-O.

\begin{tabular}{c|cc}
\hline Zustand & $U H V$ & $O$ \\
\hline$R[n m]$ & $1,69 \pm 0,05$ & $1,47 \pm 0,04$ \\
\hline
\end{tabular}


Die UHV- geglühte Probe weist einen deutlich größeren mittleren Teilchenradius als die Vergleichsprobe mit Exzeß- O auf. Da die Zwischenglühung im UHV unterhalb der Oxidationstemperatur durchgeführt wurde und die zugehörige Glühzeit von $2 \mathrm{~h}$ klein gegen die Endglühdauer von 168 h ist, können Unterschiede in der Teilchengrößenverteilung der Proben nur durch die Anwesenheit (bzw. Abwesenheit) des Exzeß-O bestimmt sein. Dies läßt den Schluß zu, daß Proben ohne Exzeß- O schneller vergröbern als Proben mit Exzeß- O.

Die Restlöslichkeit von $\mathrm{Mg}$ und $\mathrm{O}$ in der Ag- Matrix wurden aus einer bei $750{ }^{\circ} \mathrm{C}$ für 182 Tage innenoxidierten Probe ermittelt. Die Teilchen sind in diesem Zustand so groß, daß sie in sehr geringer Anzahldichte bzw. einem ausreichend großen Teilchenabstand von $\lambda_{\mathrm{V}} \approx 20 \mathrm{~nm}$ vorliegen, um die Restlöslichkeiten innerhalb einer ausscheidungsfreien Zone des rekonstruierten Volumens bestimmen zu können. Die so ermittelten Werte für die Restlöslichkeiten finden sich in Tabelle 3.4. Das Verhältnis der Mg- zu ORestlöslichkeit beträgt $(\mathrm{O} / \mathrm{Mg})_{\text {Restl. }} \approx 1,2$ und ist damit höher als das $\mathrm{O} / \mathrm{Mg}$ - Verhältnis der gesamten Probe (Tabelle 3.4). Berücksichtigt man noch eine geringere Detektionswahrscheinlichkeit für O- Atome, so erhöht sich der Wert noch merklich. Es ist also davon auszugehen, daß $\mathrm{MgO}_{\mathrm{x}}-$ Moleküle mit $\mathrm{x} \geq 1$ innerhalb der $\mathrm{Ag}$ - Matrix vorliegen.

Tabelle 3.4: Restlöslichkeit von Mg und O innerhalb der Ag-Matrix. Innere Oxidation bei $T_{I O}=750{ }^{\circ} \mathrm{C}$ für $t=182$ Tage.

\begin{tabular}{c|cc}
\hline Element & $M g$ & $O$ \\
\hline $\mathrm{c}^{0}{ }_{\mathrm{x}}[$ At. $\%]$ & $0,028 \pm 0,005$ & $0,034 \pm 0,006$ \\
\hline
\end{tabular}

\subsubsection{Untersuchung der Ag/MgO- Grenzfläche}

In [Kluthe00] konnte gezeigt werden, daß die lokale O- Konzentration der Teilchengrenzfläche höher ist als die des Mg. Aus radialen Leiterdiagrammen erkennt man einen Anstieg der relativen Sauerstoffkonzentration zur Teilchenoberfläche von ca. 40 At.\% auf bis zu 70 At.\% von der Gesamtzahl an detektierten Mg- und O-Atomen (Abb. 3.4). Dieser Anstieg kann nur durch die Anwesenheit von Exzeß- O erklärt werden 
[Kluthe00]. Aufgrund der auftretenden Meßeffekte ${ }^{6}$ konnte aus diesem GrenzflächenExzeß allerdings keine O- Belegungsdichte berechnet werden.

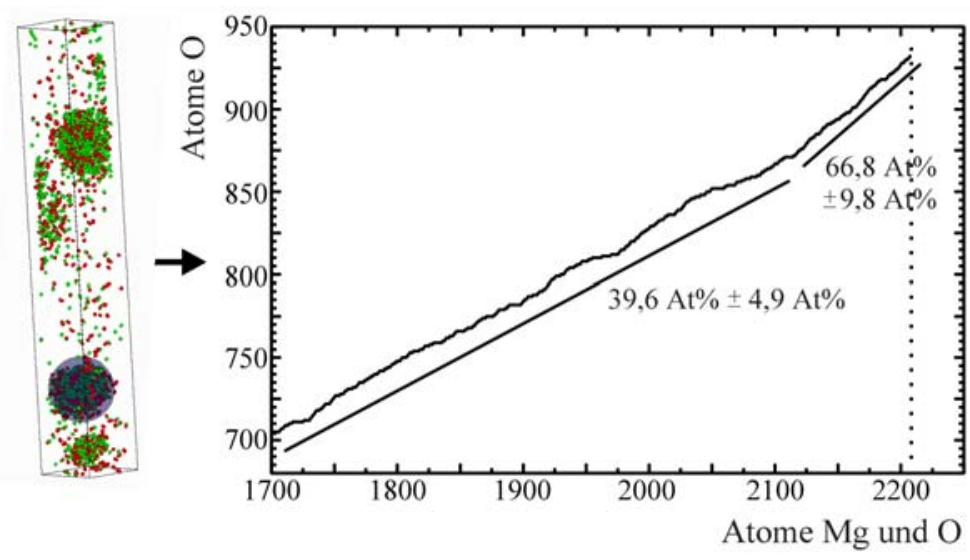

Abb. 3.4: Links: $M g O-$ Teilchen in einer Analysesphäre. Rechts: Zugehöriges radiales Leiterdiagramm vom Zentrum dieser Ausscheidung. Es wurde nur das Verhältnis von Mg- zи O-Atomen betrachtet. Die gestrichelte Linie markiert die Kugeloberfläche [KLUTHE00].

Wie in Kap. 1.2a beschrieben, kann Wasserstoff als Sonde für die Charakterisierung der M/O- Grenzfläche benutzt werden. Die segregierte Menge an Exzeß- O läßt sich danach auch indirekt durch eine H- Beladung prüfen, da anzunehmen ist, daß unter H- Angebot der schwach gebundene Exzeß- $\mathrm{O}$ zu $\mathrm{H}_{2} \mathrm{O}$ reagiert. Im TAP kann Wasserstoff erfolgreich detektiert werden, sofern die Analysen bei Meßtemperaturen tiefer als $50 \mathrm{~K}$ durchgeführt werden und die Feldverdampfung über den gesamten Apex homogen verläuft. Ist dies nicht gewährleistet, kann Restgas (bei Drücken von $10^{-8} \mathrm{~Pa}$ überwiegend $\mathrm{H}_{2} \mathrm{O}$ und $\mathrm{H}_{2}$ ) am Spitzenapex kondensieren und zusammen mit den Probenatomen analysiert werden. Bedauerlicherweise weisen $\mathrm{MgO}$ - Ausscheidungen in Ag kein homogenes Feldverdampfungsverhalten auf [KLUTHE02]. Als Folge dieser nicht homogenen Feldverdampfung tritt Wasserstoff im Massenspektrum der Analyse auf, selbst dann wenn die Probe zuvor nicht mit Wasserstoff beladen wurde [KLUTHE02]. Diese Tatsache macht es in H- beladenen Proben unmöglich, den segregierten Wasserstoff innerhalb des Analysevolumens eindeutig zu identifizieren.

6 Geringere Detektionswahrscheinlichkeit des O, sowie lokaler Vergrößerungseffekt der Oxidausscheidungen 
Motiviert durch die Annahme, daß die Feldverdampfung bei einer geschlossenen, aber sehr dünnen Oxidschicht wesentlich homogener abläuft, wurden ebenfalls Oxidschichten hergestellt. Im Rahmen dieser Arbeit gelang es dabei erstmals oxidische Schichtpakete mit Hilfe von TAP zu untersuchen (siehe Abb. 3.5).Eine detaillierte Beschreibung der TAP- Ergebnisse der unbeladenen Metall/Oxid- Schichten findet sich im Appendix in Kap. 6.2.
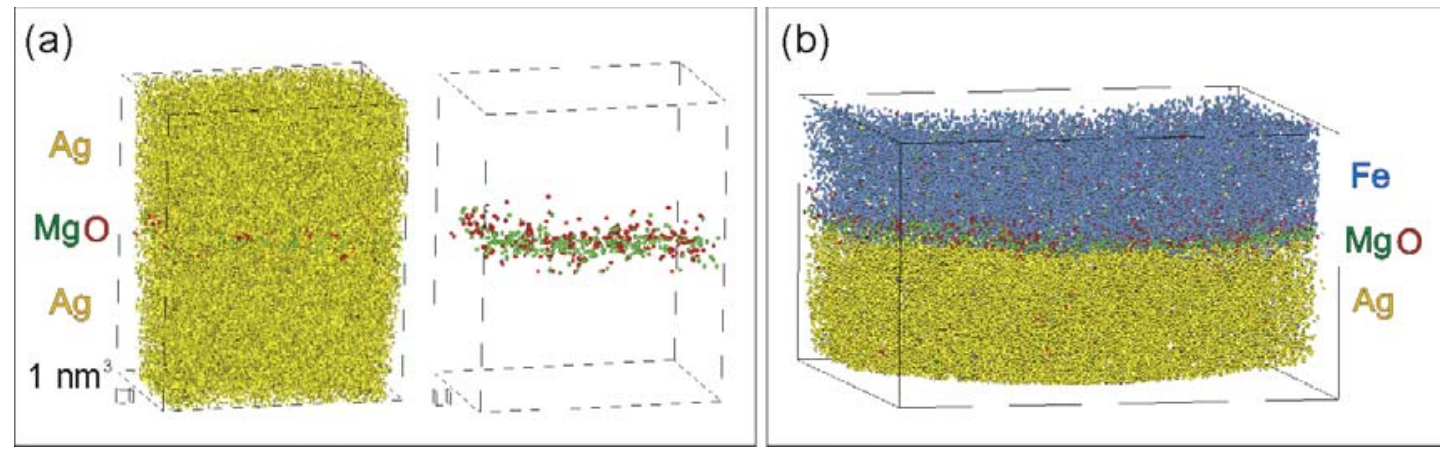

Abb. 3.5: Rekonstruierte Analysevolumina; (a) Ag/MgO/Ag-Schichtpaket, links: alle Atome, rechts: nur $\mathrm{Mg}$ - und $\mathrm{O}$ - Atome; (b) Fe/MgO/Ag-Schichtpaket. Boxgröße: $18 \times 18 \times 10,5 \mathrm{~nm}$.

Bedauerlicherweise wurde die Stabilität der Spitzen durch die Wasserstoffbeladung derart gering, daß keines der mit Deuterium beladenen $\mathrm{Ag} / \mathrm{MgO} / \mathrm{Ag}$ - Schichtpakete erfolgreich im TAP gemessen werden konnte. Alle D- beladenen Spitzen rissen immer nach einigen 10000 detektierten Ag- Ionen ab. Es muß daher festgestellt werden, daß eine Untersuchung des Segregationsverhaltens von Wasserstoff zur Charakterisierung der M/O- Grenzfläche nicht mit TAP durchgeführt werden kann. Dies gilt sowohl für $\mathrm{MgO}$ - Ausscheidungen in $\mathrm{Ag}$ als auch für $\mathrm{MgO}$ - Schichten. 


\subsection{SANS- Ergebnisse des Systems Ag/MgO}

In Kap. 3.1.2 zeigte sich, daß eine Untersuchung des Segregationsverhaltens von Wasserstoff zur Charakterisierung der M/O- Grenzfläche nicht mit TAP durchgeführt werden kann. Diese Tatsache motivierte Untersuchungen der innerlich oxidierten Systeme mit Hilfe der Neutronenkleinwinkelstreuung (SANS). Wie schon in Kap. 2.2.1 beschrieben besteht der große Vorteil dieser Methode in den sehr großen Wirkungsquerschnitten von $\mathrm{H}$, D für Neutronen. Da zudem die kohärenten Streulängen von $\mathrm{H}$ und D unterschiedliche Vorzeichen aufweisen, bietet sich die Möglichkeit einer Kontrastvariation (siehe Kap. 2.2.4) an.

\subsubsection{Unbeladene Ag/MgO- Proben}

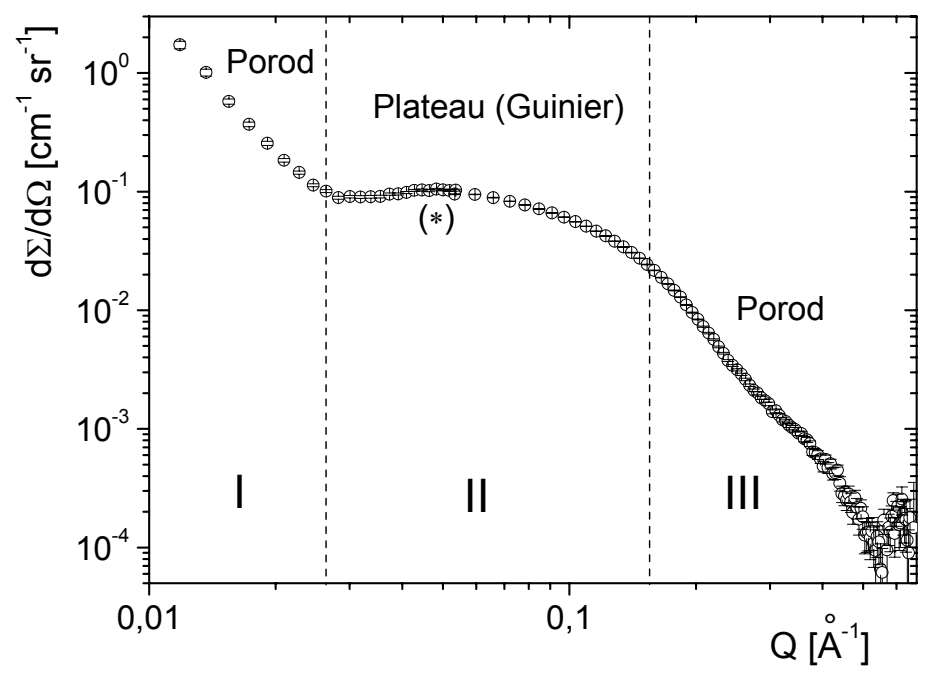

Abb. 3.6: Makroskopischer Streuquerschnitt einer bei $800{ }^{\circ} \mathrm{C}$ für $21 d$ innenoxidierten, unbeladenen Ag-1At.\%Mg-Probe. Der mittlere Radius der innerhalb der Ag-Körner gebildeten $\mathrm{MgO}$-Ausscheidungen beträgt $R_{M g O}=1,82 \pm 0,06 \mathrm{~nm}$.

In Abb. 3.6 ist eine typische Neutronenstreukurve einer unbeladenen innenoxidierten Ag-1At.\%Mg- Probe abgebildet, wie sie mittels kalter Neutronen am NG7-SANSInstrument am NIST gemessen wurde. Deutlich sind die drei Bereiche I, II und III zu erkennen. Die Bereiche I und III stellen Porod- Bereiche dar, in denen das zu erwar- 
tende $\mathrm{Q}^{-4}$ - Verhalten zu beobachten ist. Im mittleren Bereich II, dem Plateau, findet man ein Guinier- Verhalten. Da das Porod- Verhalten des Bereichs I schon bei Werten Q > $0.01 \AA^{-1}$ einsetzt, folgt aus G1. (2.12), daß die in diesem Bereich streuenden Teilchen einen Durchmesser von größer $60 \mathrm{~nm}$ besitzen. TEM- Untersuchungen zur Folge finden sich derart große MgO- Teilchen nur in den Korngrenzen des Ag wieder [GEGNER95]. Die Oxidteilchengrößen im Korn betragen nach TAP 1 bis $6 \mathrm{~nm}$. Die Porodstreuung dieser wesentlich kleineren MgO- Teilchen innerhalb der Körner entspricht daher dem Bereich III (in Abb. 3.6).

(a)

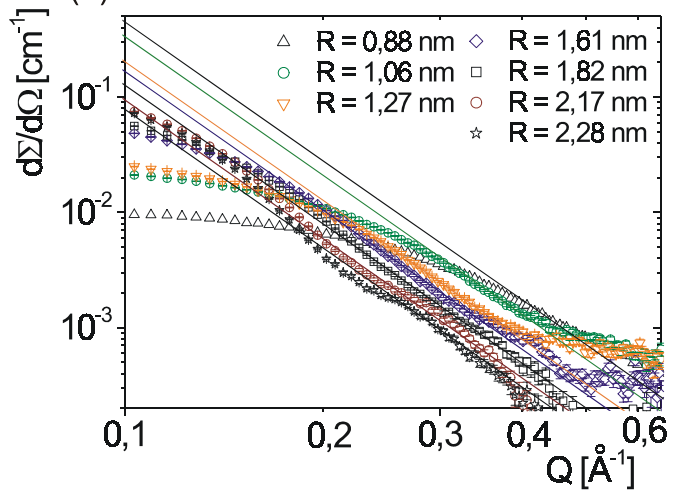

(b)

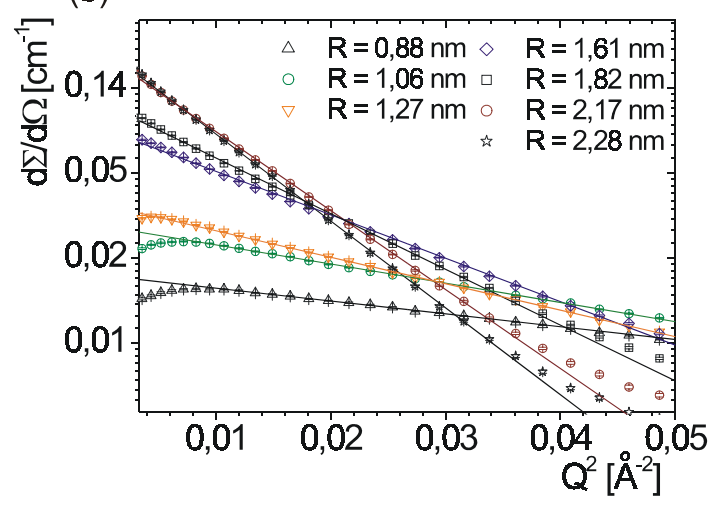

Abb. 3.7: Makroskopischer Streuquerschnitt d $\Sigma / d \Omega$ der innenoxidierten Ag-1At.\%MgProben aus Tabelle 3.5: (a) In doppeltlogarithmischer Auftragung des Bereichs III., (a) In Guinier- Auftragung innerhalb des Bereich II.; Den durchgezogenen Linien entsprechen Anpassungsgeraden nach Gl. (2.14), bzw. (b) Gl. (2.16).

Mit Hilfe von Gl. (2.16) kann innerhalb der Porod- Bereiche die totale Grenzfläche S der Teilchen bestimmt werden (siehe Abb. 3.7a). Während S im Bereich I ca. $10^{3}$ $\mathrm{cm}^{2} / \mathrm{cm}^{3}$ beträgt, findet man innerhalb der Körner Werte der Größenordnung $10^{5}$ $\mathrm{cm}^{2} / \mathrm{cm}^{3}$ (siehe Tabelle 3.5). Unter Verwendung der Gl. (2.14) wurden der Volumenbruchteil $v$ und der Teilchenradius $\mathrm{R}$ der MgO- Ausscheidungen aus dem GuinierBereich (II) bestimmt (Abb. 3.7b). Die berechneten Werte sind in Tabelle 3.5 für verschiedene Oxidationstemperaturen und Oxidationszeiten aufgelistet. Bemerkenswert ist, daß die Ausscheidungen in den Korngrenzen einen Volumenbruchteil von $\sim 20 \%$ am gesamten $\mathrm{MgO}$ ausmachen, jedoch kaum zur totalen $\mathrm{M} / \mathrm{O}$ - Grenzfläche der Probe beitragen. Der Anteil der kleinen Teilchen an der totalen M/O- Grenzfläche beträgt dagegen über $99 \%$ (vgl. Tabelle 3.5). 
Bei all diesen Proben waren zudem die Modulationen des Kugel-Formfaktors in den Streukurven zu erkennen. Nach Abziehen der Porodstreuung bei großen Q- Vektoren ist es möglich, die Maxima und Minima dieser Modulationen zu bestimmen (Abb. 3.8a). Aus diesen konnte wiederum der Teilchenradius bestimmt (siehe Kap. 2.2.3, Gl. (2.17)) und mit dem aus dem Guinier- Bereich berechneten verglichen werden.

Außerdem fällt bei genauer Betrachtung von Abb. 3.6 ein kleines Maximum (siehe (*)) innerhalb des Guinier- Plateaus bei $\mathrm{Q} \sim 0,045 \AA^{-1}$ auf. Dieses Maximum rührt von dem sog. Strukturfaktor S(Q) einer Teilchen- Teilchen Korrelation her. Nach Abziehen des Porod- Beitrages der Teilchen in den Korngrenzen, sowie des Guinier- Beitrages der Teilchen innerhalb der Körner, läßt sich die Lage des Maximums $\mathrm{Q}_{\mathrm{k}}$ bestimmen $(\mathrm{Abb}$. 3.8b). Aus $\mathrm{Q}_{\mathrm{k}}$ kann dann aus dem Zusammenhang $\lambda_{\mathrm{k}}=2 \pi / \mathrm{Q}_{\mathrm{k}}$ der mittlere Abstand der Teilchen berechnet werden [GLATTER83]. Zum Vergleich wird der Teilchenabstand $\lambda_{v}$ ebenfalls aus der Anzahldichte $\mathrm{N}_{\mathrm{v}}$ der $\mathrm{MgO}$ - Ausscheidungen, bzw. aus deren mittleren Radius R sowie dem Volumenbruchteil $v$, berechnet und in Tabelle 3.5 den Werten $\lambda_{\mathrm{k}}$ gegenübergestellt. $\lambda_{v}$ folgt aus Gleichung (3.1):

$$
\lambda_{v}=\frac{1}{\sqrt[3]{N_{P}}}=\frac{R}{\sqrt[3]{3 v / 4 \pi}}
$$

(a)

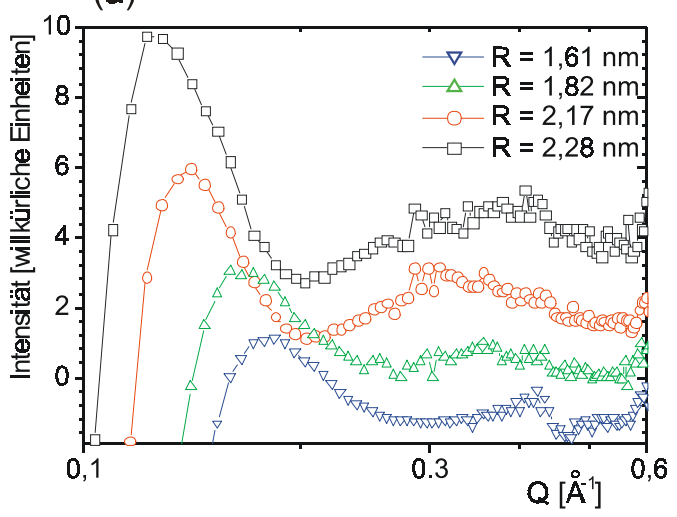

(b)

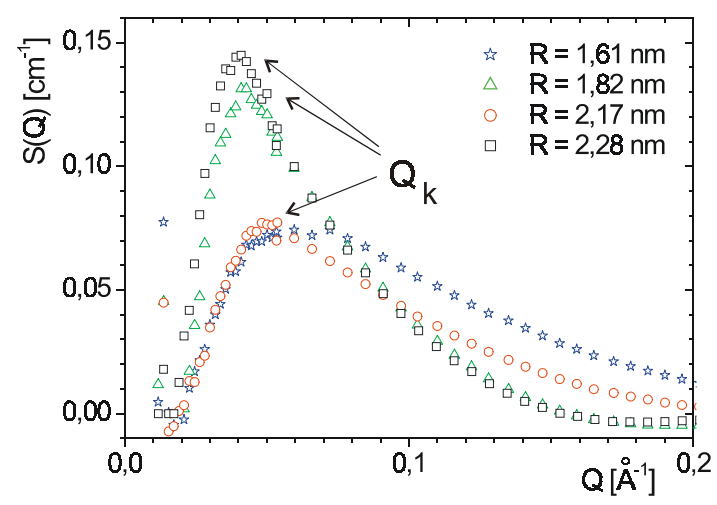

Abb. 3.8: (a) Modulationen des Kugel-Formfaktors der MgO-Teilchen im Bereich großer Q-Vektoren; (b) Verlauf des Strukturfaktors $S(Q)$ der Teilchen- Teilchen Korrelation für Proben mit unterschiedlichen Teilchenradien $R$. 
Durch die Innere Oxidation der binären AgMg- Legierung werden $\mathrm{MgO}$ - Ausscheidungen von 0,88 bis 2,28 nm Teilchenradius gebildet. Die Größe der Ausscheidungen nimmt offensichtlich mit zunehmender Oxidationstemperatur $\mathrm{T}_{\mathrm{IO}}$, sowie mit steigender Glühdauer $t_{I O}$, zu. Innerhalb der Fehlergrenzen stimmen die aus dem Guinier- Bereich ermittelten mittleren Teilchenradien mit den aus den Modulationen bestimmten Radien überein. Aufgrund der geringeren Fehler wurden für die weitere Auswertung die aus der Guinierstreuung bestimmten Teilchenradien verwendet.

Tabelle 3.5: Aus SANS- bestimmte Werte für $\mathrm{MgO}$ - Teilchen innerhalb der AgKörner. $T_{I O}$ ist die Oxidationstemperatur, $t_{I O}$ die Oxidationsdauer.

\begin{tabular}{l|llllll}
\hline$T_{I O} / t_{I O}$ & $v[$ Vol.\%] & $R_{\text {Guinier }}[\mathrm{nm}]$ & $R_{\text {Modul. }}[\mathrm{nm}]$ & $S\left[10^{5} \mathrm{~cm}^{-1}\right]$ & $\lambda_{k}[\mathrm{~nm}]$ & $\lambda_{v}[\mathrm{~nm}]$ \\
\hline $650{ }^{\circ} \mathrm{C} / 1 \mathrm{~d}$ & $0,83 \pm 0,06$ & $0,88 \pm 0,01$ & $0,91 \pm 0,07$ & $7,81 \pm 0,05$ & $6,1 \pm 0,2$ & $7,0 \pm 0,1$ \\
$750{ }^{\circ} \mathrm{C} / 1 \mathrm{~d}$ & $0,85 \pm 0,06$ & $1,06 \pm 0,01$ & $1,09 \pm 0,06$ & $5,66 \pm 0,05$ & $6,5 \pm 0,2$ & $8,4 \pm 0,1$ \\
$750{ }^{\circ} \mathrm{C} / 14 \mathrm{~d}$ & $0,67 \pm 0,07$ & $1,27 \pm 0,02$ & $1,27 \pm 0,08$ & $4,93 \pm 0,05$ & $7,4 \pm 0,3$ & $11,0 \pm 0,1$ \\
$750{ }^{\circ} \mathrm{C} / 21 \mathrm{~d}$ & $0,76 \pm 0,07$ & $1,61 \pm 0,02$ & $1,59 \pm 0,14$ & $3,74 \pm 0,04$ & $13,2 \pm 0,4$ & $12,6 \pm 0,5$ \\
$800^{\circ} \mathrm{C} / 21 \mathrm{~d}$ & $0,71 \pm 0,07$ & $1,82 \pm 0,02$ & $1,78 \pm 0,12$ & $3,11 \pm 0,04$ & $15,3 \pm 0,5$ & $14,0 \pm 0,3$ \\
$850^{\circ} \mathrm{C} / 3 \mathrm{~d}$ & $0,76 \pm 0,07$ & $2,17 \pm 0,02$ & $2,15 \pm 0,19$ & $2,82 \pm 0,04$ & $17,8 \pm 0,7$ & $15,0 \pm 0,4$ \\
$825{ }^{\circ} \mathrm{C} / 21 \mathrm{~d}$ & $0,70 \pm 0,06$ & $2,28 \pm 0,02$ & $2,28 \pm 0,15$ & $2,24 \pm 0,04$ & $19,2 \pm 0,7$ & $16,1 \pm 0,4$ \\
$835^{\circ} \mathrm{C} / 21 \mathrm{~d}$ & $0,71 \pm 0,07$ & $2,38 \pm 0,02$ & $2,35 \pm 0,16$ & $2,15 \pm 0,04$ & $20,6 \pm 0,7$ & $20,7 \pm 0,4$ \\
\hline
\end{tabular}

Trägt man die aus SANS bestimmte Grenzfläche $\mathrm{S}_{\mathrm{MgO}}$ der $\mathrm{MgO}$ - Ausscheidungen gegen deren reziproken Teilchenradius $\mathrm{R}$ auf, findet man generell eine gute Übereinstimmung mit dem Verlauf der zugehörigen Ursprungsgerade (Abb. 3.9). Die totale Grenzfläche $\mathrm{S}_{\mathrm{MgO}}$ der Teilchen wird dabei entsprechend Gleichung (3.2), die einen konstanten Volumenbruchteil der Oxidphase voraussetzt $(v=$ konst.), mit kleinerem Teilchenradius R, bzw. mit abnehmender Anzahldichte $\mathrm{N}_{\mathrm{V}}$ zunehmend größer (siehe Abb. 3.9):

$\mathrm{S}_{\mathrm{MgO}}=4 \pi \mathrm{R}^{2} \cdot \mathrm{N}_{\mathrm{V}}=\frac{3 \mathrm{v}}{\mathrm{R}}$, da $\mathrm{v}=\frac{4}{3} \pi \mathrm{R}^{3} \cdot \mathrm{N}_{\mathrm{V}}$ (Massenerhaltung) 


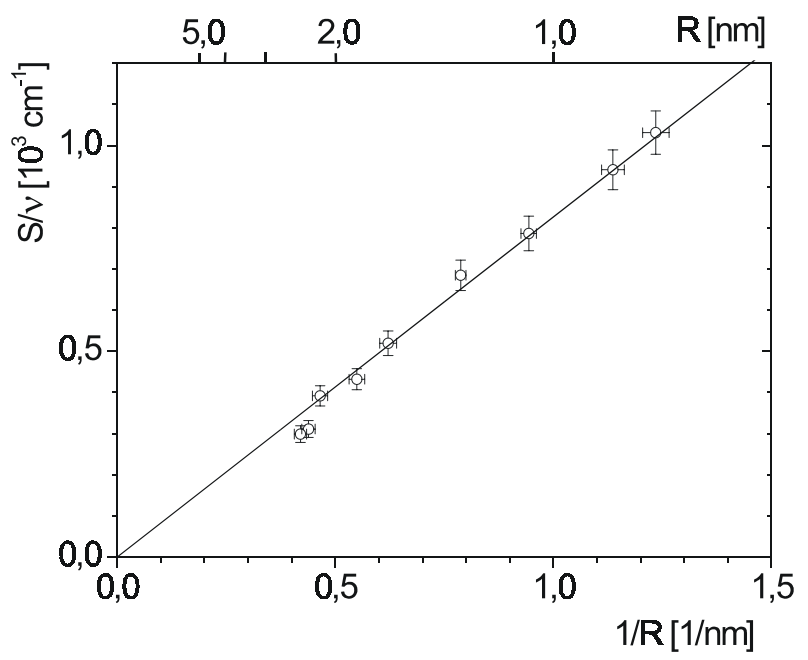

Abb. 3.9: Auftragung $S_{M g O} / \nu_{M g O}$ gegen den reziproken Teilchenradius. Die Werte entsprechen dem Verlauf einer Ursprungsgerade. 


\subsubsection{H- und D- beladene Ag/MgO- Proben}
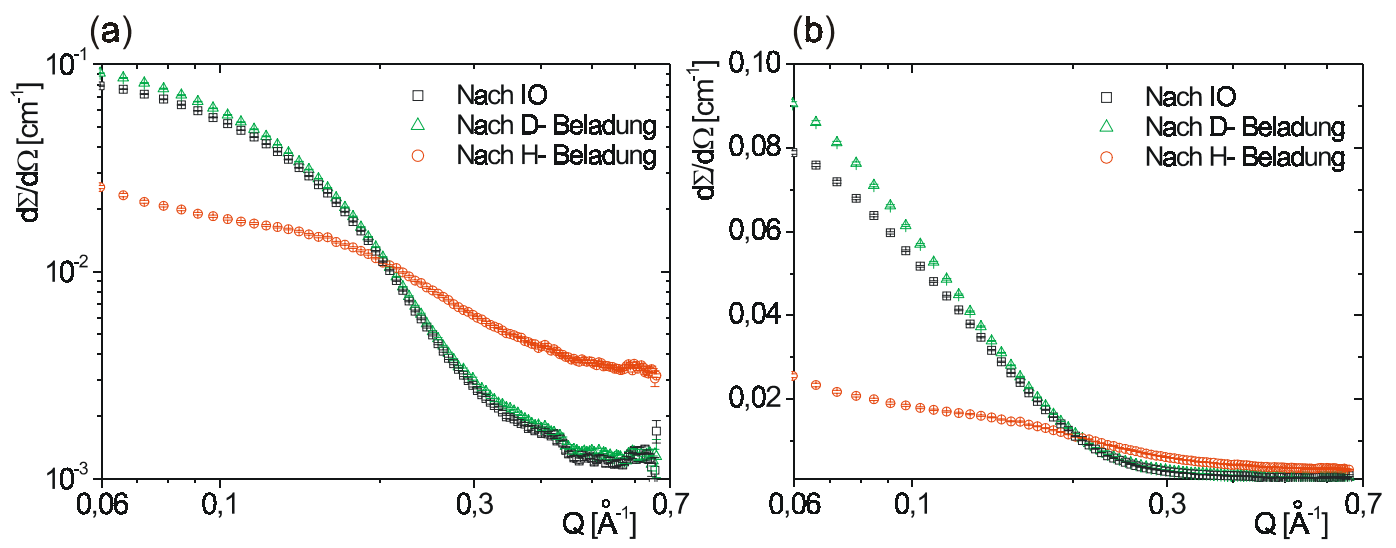

Abb. 3.10: (a) Doppeltlogarithmische Auftragung von $d \Sigma / d \Omega(Q)$ einer unbeladenen, einer mit $\mathrm{H}$ - und einer mit D beladenen innenoxidierten Ag-1At.\%Mg-Probe. Der mittlere Teilchenradius beträgt $R_{M g O}=1,61 \pm 0,05 \mathrm{~nm}$. (b) Halblogarithmische Auftragung von (a).

Abb. 3.10 zeigt exemplarisch die Veränderung des Streuverhaltens einer innenoxidierten Ag-1At.\%Mg- Probe bei H- Beladung sowie bei D- Beladung. Die Streukurven wurden für zwei identische, innenoxidierte Proben aufgenommen, die zunächst beide im unbeladenen Zustand gemessen wurden und von denen anschließend eine Probe mit $\mathrm{H}$ und die andere Probe mit $\mathrm{D}$ beladen wurde. Hatten diese beiden Proben noch ein identisches Streuverhalten vor der Beladung, wiesen sie nach der Beladung ein stark unterschiedliches Streuverhalten auf. Die aus der negativen kohärenten Streulänge des Wasserstoff $\left(b_{H}=-3,74 \mathrm{fm}\right.$, siehe Tabelle 2.1) resultierende Abnahme der gestreuten Intensität ist deutlich zu erkennen. Die Abnahme entspricht ca. $70 \%$ im Verhältnis zur unbeladenen Probe. Eine ca. 15 \%ige Zunahme ist dagegen bei der deuterierten Probe $\left(b_{\mathrm{D}}=+6,67 \mathrm{fm}\right)$ festzustellen. Dies ist besonders gut in einer halblogarithmischen Auftragung zu erkennen (siehe Abb. 3.10b). Selbst nach einer Glühung der beladenen Proben im Hochvakuum ( 10 $\left.0^{-2} \mathrm{~Pa}\right)$ zeigte sich immer noch ein identisches Streuverhalten. Durch den im Vergleich zu D sehr viel größeren inkohärenten Streuquerschnitt des H (vgl. Tabelle 2.1) weist die H- beladenen Probe zudem eine sehr viel höhere Untergrundintensität auf. Mit Hilfe der Gl. (2.11) kann aus diesem Beitrag der inkohärenten Steuung die H- Konzentration der Proben bestimmt werden. Für Proben, 
mit unterschiedlichen mittleren Teilchenradien der $\mathrm{MgO}-$ Ausscheidungen, sind so die $\mathrm{H}$ - Konzentrationen ermittelt und in Tabelle 3.6 aufgelistet worden.

Zur genaueren Charakterisierung der vermuteten H (bzw. D)- Schale wurde daher aus einer modifizierten Guinier- Auftragung (siehe Kap. 2.2.4) der Streukurven von H- bzw. D- beladenen Proben der sog. Schalenkontrast $\Delta \rho_{\mathrm{S}} \cdot \delta \mathrm{R}$ ermittelt. Der gesuchte Schalenkontrast $\Delta \rho_{S} \cdot \delta$ R läßt sich nach G1. (2.20) in dieser Auftragung aus dem Schnittpunkt von y- Achse und der Anpassungsgerade des Guinier- Bereichs bestimmen (siehe Abb. 3.11). Die so berechneten Werte sind für verschiedene Proben zusammen mit den entsprechenden H- Konzentrationen gegen die aus den unbeladenen Proben ermittelten MgO- Teilchengrößen in Tabelle 3.6 aufgelistet.
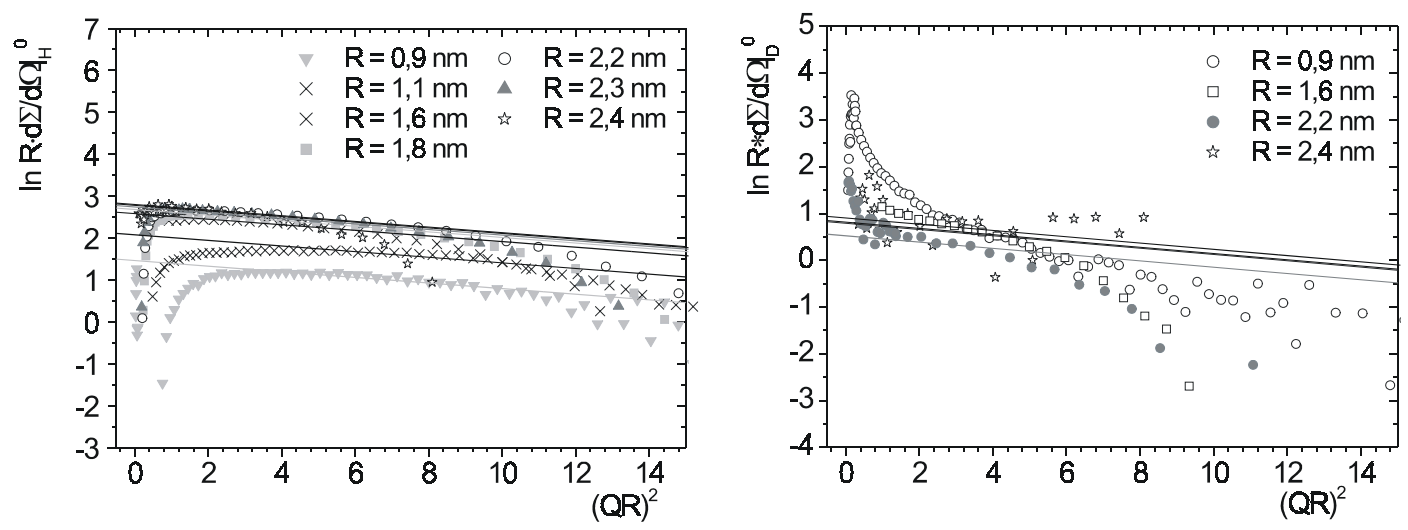

Abb. 3.11: Modifizierte Guinier- Plots von $R \cdot d \Sigma /\left.d \Omega\right|_{S}{ }^{0}$ gegen $(Q R)^{2}$ innerlich oxidierter Ag/MgO-Proben. Links: H- beladene Proben, Rechts: D- beladene Proben. Den durchgezogenen Linien entsprechen Anpassungsgeraden für den Guinierbereich (bei $Q^{2} R^{2} \approx 3$ ) nach Gl. (2.20).

Für Teilchen $\mathrm{R} \geq 1,6 \mathrm{~nm}$ können folgende Mittelwerte für den Schalenkontrast angegeben werden:

Für H- beladene Ag/MgO- Proben: $\left\langle\Delta \rho_{S} \delta \mathrm{R}>_{\mathrm{H}}=-650 \pm 83 \mathrm{~cm}^{-1}\right.$.

Für D- beladene $\mathrm{Ag} / \mathrm{MgO}$ - Proben: $\left\langle\Delta \rho_{\mathrm{S}} \delta \mathrm{R}>_{\mathrm{D}}=+88 \pm 23 \mathrm{~cm}^{-1}\right.$

Der Schalenkontrast für Wasserstoff ist also wesentlich größer als für Deuterium. Dies ist erstaunlich, da die kohärente Streulänge von D vom Betrag größer ist als die von $\mathrm{H}$ 
(vgl. Tabelle 2.1). Im Falle einer reinen Segregation (d.h. ohne Änderung der Matrixdichte) würde man den absolut größeren Kontrast für D (und nicht für H) erwarten. Da für Deuterium wie für Wasserstoff die gleiche Schalendicke $\delta \mathrm{R}$ anzunehmen ist, bedeutet dieses Ergebnis, daß der Kontrast für Deuterium um einen zusätzlichen Term vermindert, bzw. der von Wasserstoff verstärkt wird. Außerdem ist bemerkenswert, daß für Teilchen $\mathrm{R}<1,6 \mathrm{~nm}$ der Schalenkontrast abnimmt (Tabelle 3.6). Dieses Verhalten zeigt sich besonders deutlich bei den $\mathrm{H}$ - beladenen $\mathrm{Ag} / \mathrm{MgO}$ - Proben.

Tabelle 3.6: Schalenkontrast $\Delta \rho_{S} \cdot \delta R$ nach $H$ - bzw. D-Beladung, sowie aus der inkohärenten Streuung bestimmte H-Konzentrationen der Ag/MgO-Proben.

\begin{tabular}{l|lll}
\hline$R[\mathrm{~nm}]$ & $\left(\Delta \rho_{S} \cdot \delta R\right)_{H}\left[\mathrm{~cm}^{-1}\right]$ & $\left(\Delta \rho_{S} \cdot \delta R\right)_{D}\left[\mathrm{~cm}^{-1}\right]$ & $c_{H}[\mathrm{At} . \%]$ \\
\hline $0,88 \pm 0,01$ & $-189 \pm 15$ & $+113 \pm 18$ & $0,30 \pm 0,02$ \\
$1,27 \pm 0,01$ & $-356 \pm 16$ & k.A. & $0,58 \pm 0,02$ \\
$1,61 \pm 0,05$ & $-567 \pm 18$ & $+95 \pm 7$ & $0,48 \pm 0,03$ \\
$1,82 \pm 0,06$ & $-618 \pm 21$ & k.A. & $0,48 \pm 0,03$ \\
$2,17 \pm 0,08$ & $-697 \pm 26$ & $+65 \pm 5$ & $0,32 \pm 0,03$ \\
$2,28 \pm 0,08$ & $-678 \pm 24$ & k.A. & $0,32 \pm 0,03$ \\
$2,38 \pm 0,09$ & $-688 \pm 31$ & $+103 \pm 15$ & k.A. \\
\hline
\end{tabular}

Da sich Wasserstoff und Deuterium chemisch identisch verhalten, sollten auch die Belegungsdichten dieser Elemente an der $\mathrm{Ag} / \mathrm{MgO}$ - Grenzfläche identisch sein, womit gilt: $\Theta_{\mathrm{H}}=\Theta_{\mathrm{D}}$. Über Gleichung (2.21) kann somit bei Proben gleicher MgO- Teilchengrößenverteilung mit Hilfe einer H/D- Kontrastvariation die Belegungsdichte des Wasserstoffs $\Theta_{\mathrm{H}}$ an der Teilchengrenzfläche bestimmt werden (siehe Kap. 2.2.4). Abb. 3.12 zeigt modifizierte Guinier- Plots der Differenz R.d $\Sigma /\left.\mathrm{d} \Omega\right|^{0}{ }^{\mathrm{D}-\mathrm{H}}$ nach Gl. (2.21) für die Proben aus Kap. 3.2.2, aus denen die Belegungsdichte $\Theta_{\mathrm{H}}$ bestimmt wurde. 


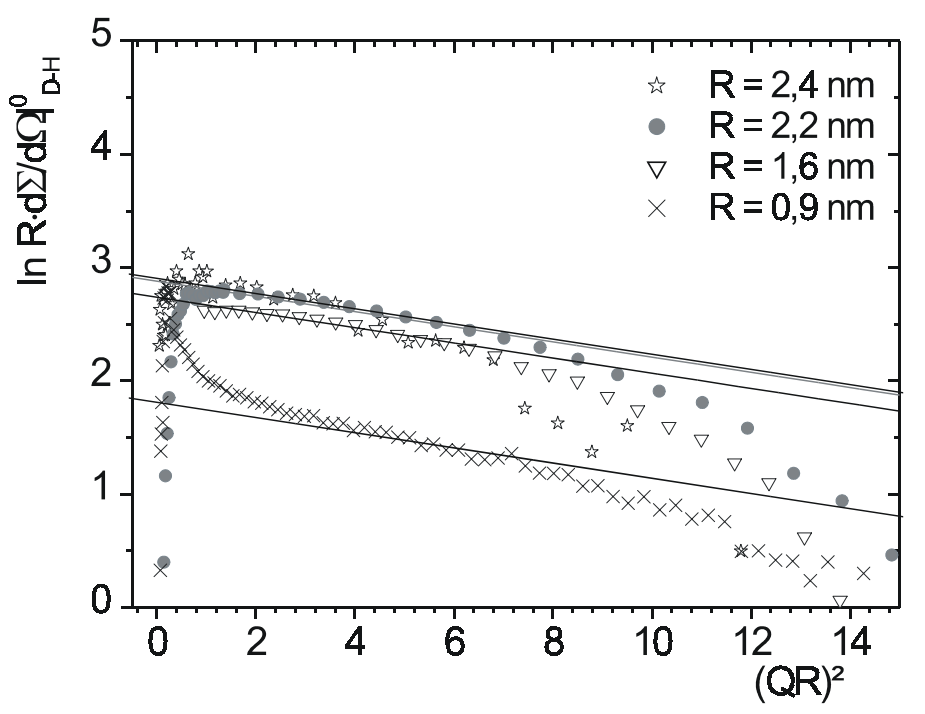

Abb. 3.12: Modifizierte Guinier- Auftragung der Differenz $R \cdot d \Sigma /\left.d \Omega\right|^{0}{ }_{D-H} \quad$ der normierten Nettostreuquerschnitte für $D$ und $H$ nach Gl. (2.21). Die Belegungsdichte $\Theta_{H}$ wird aus dem Achsenabschnitt der Anpassungsgeraden bestimmt.

Für verschiedene Teilchenradien liefert diese Auswertung folgende Werte für die HBelegungsdichte der Ag/MgO- Teilchengrenzfläche (Tabelle 3.7):

Tabelle 3.7: H-Belegungsdichten der Ag/MgO-Teilchengrenzfläche für verschiedene Teilchenradien $R$.

\begin{tabular}{c|c}
\hline$R[\mathrm{~nm}]$ & $\Theta_{H}\left[\mathrm{~cm}^{-2}\right]$ \\
\hline $0,88 \pm 0,01$ & $(2,6 \pm 0,2) \cdot 10^{14}$ \\
$1,61 \pm 0,05$ & $(6,5 \pm 0,2) \cdot 10^{14}$ \\
$2,17 \pm 0,08$ & $(7,3 \pm 0,3) \cdot 10^{14}$ \\
$2,38 \pm 0,09$ & $(7,5 \pm 0,5) \cdot 10^{14}$ \\
\hline
\end{tabular}

Für Teilchen $\mathrm{R} \geq 1,61 \mathrm{~nm}$ berechnet sich eine mittlere Belegungsdichte von $\Theta_{\mathrm{H}}=(7,1 \pm 0,4) \cdot 10^{14} \mathrm{~cm}^{-2}$ 
Wie in Kap. 4 noch gezeigt wird, entspricht dieser Wert einer halben Monolage Wasserstoff (bzw. Deuterium) an der $\mathrm{Ag} / \mathrm{MgO}$ - Teilchengrenzfläche. Für Teilchen mit $\mathrm{R}=$ $0,88 \mathrm{~nm}$ findet man ein abweichendes Verhalten. Die H- Belegungsdichte beträgt bei diesen kleinsten Teilchen nur etwa ein Drittel der Belegungsdichte der größeren Teilchen. Diese interessante Tatsache wird ebenfalls in Kap. 4 diskutiert.

Mit Kenntnis der H- Belegungsdichten $\Theta_{\mathrm{H}}$ (Tabelle 3.7), sowie des Schalenkontrastes $<\Delta \rho_{\mathrm{S}} \delta \mathrm{R}>$ von $\mathrm{H}$ und D (Tabelle 3.6) kann nun die Änderung der Ag- Dichte durch Segregation mit Hilfe der Formel (2.19) berechnet werden (siehe Tabelle 3.8). Für Teilchen $\mathrm{R} \geq 1,6 \mathrm{~nm}$ entspricht die Dichteänderung etwa der Menge segregierter Wasserstoff- Atome. Bei kleinsten Teilchen mit $\mathrm{R}=0,88 \mathrm{~nm}$ findet sich eine geringere Ag- Dichteänderung $\Delta \Theta_{\mathrm{Ag}}$ von nur etwa $50 \%$ der H- Belegungsdichte $\Theta_{\mathrm{H}}$.

Tabelle 3.8: Änderung der Ag-Matrixdichte durch Wasserstoff- Segregation. Zum Vergleich ist die Wasserstoff- Belegungsdichte mit aufgeführt.

\begin{tabular}{c|c|c|c}
\hline$R[\mathrm{~nm}]$ & $\Delta \Theta_{A g}\left[\mathrm{~cm}^{-2}\right]$ & $\Theta_{H}\left[\mathrm{~cm}^{-2}\right]$ & $\Delta \Theta_{A g} / \Theta_{H}$ \\
\hline $0,88 \pm 0,01$ & $(1,3 \pm 0,3) \cdot 10^{14}$ & $(2,6 \pm 0,2) \cdot 10^{14}$ & $0,50 \pm 0,12$ \\
$1,61 \pm 0,05$ & $(5,7 \pm 0,3) \cdot 10^{14}$ & $(6,5 \pm 0,2) \cdot 10^{14}$ & $0,88 \pm 0,05$ \\
$2,17 \pm 0,08$ & $(7,1 \pm 0,4) \cdot 10^{14}$ & $(7,3 \pm 0,3) \cdot 10^{14}$ & $0,97 \pm 0,07$ \\
$2,38 \pm 0,09$ & $(6,8 \pm 0,6) \cdot 10^{14}$ & $(7,5 \pm 0,5) \cdot 10^{14}$ & $0,91 \pm 0,10$ \\
\hline
\end{tabular}

\subsubsection{Experimente an UHV- entgasten Ag/MgO- Proben}

Um den Nachweis der strukturellen Leerstellen an der $\{111\}$ - orientierten M/O- Grenzfläche und der damit verbundenen Segregation von Exzeß- O zu führen, wurde die Änderung des Streuverhaltens von innenoxidierten Ag-1At.\%Mg- Proben nach einer UHV- Entgasung bei $700{ }^{\circ} \mathrm{C}$ für $2 \mathrm{~h}$ untersucht. 


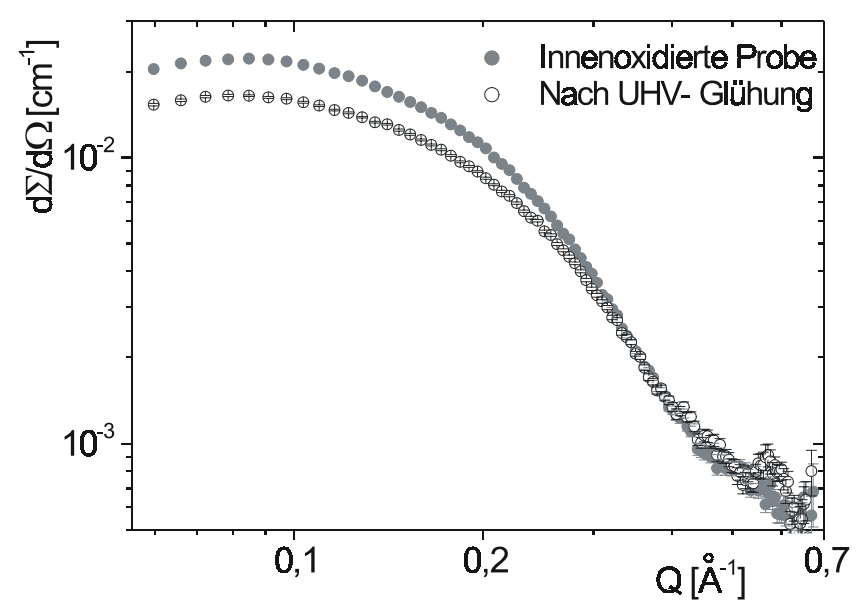

(a)

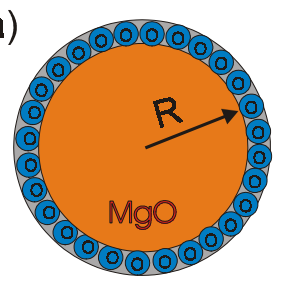

(b)

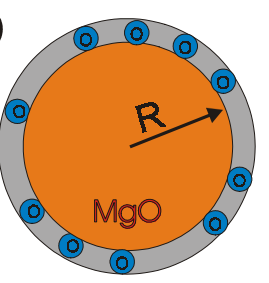

Abb. 3.13: Doppeltlogarithmische Auftragung $d \Sigma / d \Omega(Q)$ einer innenoxidierten Ag1 At.\%Mg-Probe vor und nach Glühung im UHV, $R_{M g O}=1 \mathrm{~nm}$; Rechts: Skizze zum KS-Modell, (a) Nach IO (b) Nach UHV-Glühung.

Abb. 3.13 zeigt das Streuverhalten einer UHV- entgasten Probe im Vergleich zu dem einer innenoxidierten Probe. Man erkennt eine deutliche Abnahme der gestreuten Intensität innerhalb des Guinier- Bereichs. Unter der Annahme, daß die Abnahme der gestreuten Intensität aus einer Desorbtion von Exzeß-O resultiert, kann das KugelSchale Modell auch hier angewendet werden (vgl. Abb. 3.13a,b). Die UHV- entgaste Probe entspricht dabei $\mathrm{d} \Sigma /\left.\mathrm{d} \Omega\right|_{\mathrm{K}}$ und $\mathrm{d} \Sigma /\left.\mathrm{d} \Omega\right|_{\mathrm{K}+\mathrm{S}}$ der frisch innenoxidierten Probe. Analog wie für den Wasserstoff in Kap. 3.2.2 läßt sich über Gleichung (2.20) das Produkt aus Kontrast $\Delta \rho_{\text {Ex-O }}$ und Dicke $\delta R$ der segregierten Schale Exzeß- O bestimmen. Nach dem strukturellen Modell von Huang, Mader und Kirchheim (siehe Kap. 1.2.1) bildet sich bei der Desorbtion eines Exzeß-O eine strukturelle Leerstelle [HUANG91], d.h. die Matrixdichte an der Grenzfläche wird nicht wesentlich verändert. Entsprechend diesem Modell ist folgender Kontrast für eine Schale Exzeß-O anzunehmen:

$$
\left(\Delta \rho_{\mathrm{S}} \cdot \delta \mathrm{R}\right)_{\mathrm{Ex} . \mathrm{O}}=\Delta \Theta_{\text {Ex.O }} \cdot \mathrm{b}_{\mathrm{O}} \cdot
$$

Als Teilchenradius wird der der UHV- entgasten Probe verwendet (siehe Abb. 3.13b). 
Tabelle 3.9: Bestimmte Werte für Abnahme des Teilchenradius infolge der UHVEntgasung: Schalenkontrast und daraus errechnete Belegungsdichte des Exzeß-O unter Annahme von Gl. (3.3).

\begin{tabular}{cccc}
\hline$R_{I O}[\mathrm{~nm}]$ & $R_{U H V}[\mathrm{~nm}]$ & $(\Delta \rho \cdot \delta R)_{E x-O}\left[\mathrm{~cm}^{-1}\right]$ & $\Delta \Theta_{E x-O}\left[\mathrm{~cm}^{-2}\right]$ \\
\hline $1,06 \pm 0,01$ & $1,00 \pm 0,01$ & 150 & $2,59 \cdot 10^{14}$ \\
$1,27 \pm 0,01$ & $1,24 \pm 0,01$ & 66 & $1,14 \cdot 10^{14}$ \\
$2,38 \pm 0,02$ & $2,32 \pm 0,02$ & 15 & $0,25 \cdot 10^{14}$ \\
\hline
\end{tabular}

Wie in Tabelle $3.9 \mathrm{zu}$ erkennen, findet man bei allen untersuchten Proben nach der Glühung im UHV einen geringeren Teilchenradius. Die durchschnittliche Abnahme des Teilchenradius $\mathrm{R}$ beträgt $\delta \mathrm{R}=0,5 \pm 0,1 \AA$. Erstaunlicherweise ist der Kontrast der Schale allerdings nicht konstant, sondern nimmt mit zunehmendem Teilchenradius ab. Eine Berechnung der Desorbtionsdichte $\Delta \Theta_{\text {Ex.O }}$ nach Gl. (3.3) liefert daher kleinere Belegungsdichten mit zunehmenden Teilchenradius. Möglicherweise wird der Kontrast der Schale durch einen zusätzlichen Streubeitrag, wie bei der H- Segregation, vermindert. Im Fall des Sauerstoffs ist es leider nicht möglich, analog zum Wasserstoff eine Kontrastvariation zur Identifizierung dieses Streubeitrages durchzuführen.

Es ist allerdings möglich, allein aus der Änderung der Teilchengröße $\delta \mathrm{R}$ durch UHVEntgasung, die Desorbtionsdichte $\Delta \Theta_{\text {Ex.O }}$ abzuschätzen. Mit einer Schalendicke von $\delta R$ $=0,5 \AA$, sowie dem Atomvolumen des Sauerstoffs von $\Omega_{\mathrm{O}}=23,25 \AA^{3}$, errechnet sich eine mittlere Desorbtionsdichte $\Delta \Theta_{\text {Ex.O }}$ von

$\Delta \Theta_{\text {Ex.O }}=\delta R / \Omega_{\mathrm{O}}=(2,2 \pm 0,5) \cdot 10^{14} \mathrm{O} / \mathrm{cm}^{2}$.

Der ermittelte Wert für $\Delta \Theta_{\text {Ex.O }}$ entspricht allerdings nur dann der Exzeß-O- Belegungs-

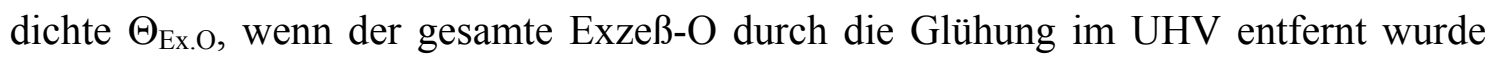
(vgl. Abb. 3.13). 
(a)

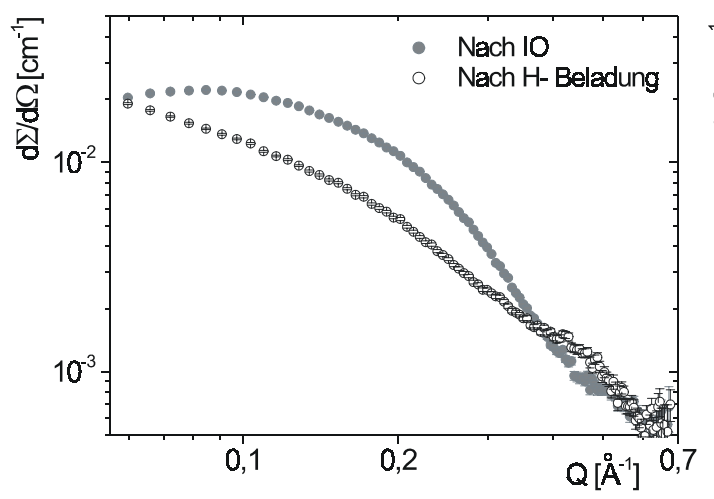

(b)

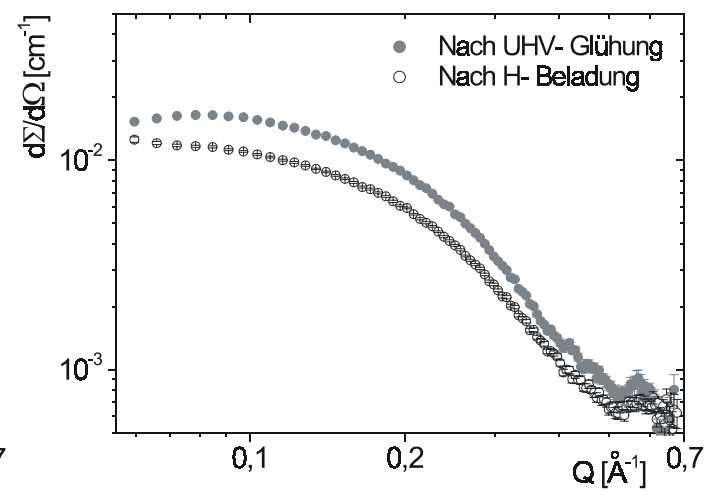

Abb. 3.14: Änderung des Streuverhaltens einer IO Ag-1At.\%Mg-Probe bei HBeladung; $R=1 \mathrm{~nm}$ (a) direkt nach der IO (b) nach UHV-Glühung. Die IO-Probe zeigt eine deutlich stärkere Abnahme in $d \Sigma / d \Omega$ nach $H$-Beladung.

Um den Zusammenhang von Exzeß- O und segregiertem Wasserstoff experimentell nachzuweisen, wurde das H- und D- Sorbtionsverhalten UHV- entgaster Proben untersucht. Wie in Abb. 3.14 zu erkennen, ist die Abnahme in $\mathrm{d} \Sigma / \mathrm{d} \Omega$ nach $\mathrm{H}$ - Beladung bei frisch innenoxidierten Proben größer als bei UHV- entgasten Proben. Die H- Belegungsdichte sowie die Ag- Verdrängung wurde für beide Proben mit Hilfe der H/DKontrastvariation analog zu Kap. 3.2.2 bestimmt (Tabelle 3.10):

Tabelle 3.10: H-Belegungsdichte $\Theta_{H}$ und Ag-Verdrängung $\Delta \Theta_{A g}$ von innenoxidierten Proben und UHV-geglühten Proben; $R=2,38 \mathrm{~nm}$.

\begin{tabular}{cccc}
\hline$\Theta_{H}^{I O}\left[10^{14} \mathrm{~cm}^{-2}\right]$ & $\Theta_{H}{ }^{U H V}\left[10^{I 4} \mathrm{~cm}^{-2}\right]$ & $\Delta \Theta_{A g}^{I O}\left[10^{14} \mathrm{~cm}^{-2}\right]$ & $\Delta \Theta_{A g}{ }^{U H V}\left[10^{I 4} \mathrm{~cm}^{-2}\right]$ \\
\hline$(7,5 \pm 0,5) \cdot 10^{14}$ & $(3,0 \pm 0,3) \cdot 10^{14}$ & $(6,8 \pm 0,6) \cdot 10^{14}$ & $(2,9 \pm 0,4) \cdot 10^{14}$ \\
\hline
\end{tabular}

Beim Vergleich der Belegungsdichten zeigt sich, daß die frisch IO Probe mehr als doppelt so viel H- sorbiert wie die UHV geglühte Probe. Die Abnahme der Belegungsdichte infolge der UHV- Glühung beträgt:

$\Delta \Theta_{\mathrm{H}}=(4,5 \pm 0,6) \cdot 10^{14} \mathrm{~cm}^{-2}$.

Außerdem zeigt sich, daß die Änderung der Ag- Matrixdichte auch nach einer UHVGlühung der Menge an segregiertem Wasserstoff entspricht, also $\Theta_{\mathrm{H}} \approx \Delta \Theta_{\mathrm{Ag}}$ gilt. 


\subsection{TAP-Ergebnisse des Systems $\mathrm{Ag} / \mathrm{Mn}_{3} \mathrm{O}_{4}$}

Im Rahmen dieser Arbeit wurde mit $\mathrm{Ag} / \mathrm{Mn}_{3} \mathrm{O}_{4}$ ein weiteres Metall/Oxid- System untersucht. Im Vergleich zu MgO- Ausscheidungen weisen $\mathrm{Mn}_{3} \mathrm{O}_{4^{-}}$Teilchen in SilberMatrix einige interessante Unterschiede auf. Bei der Inneren Oxidation einer Ag-MnLegierung bilden sich $\mathrm{Mn}_{3} \mathrm{O}_{4^{-}}$Oktaeder, die nur $\{111\}$ - orientierte Grenzflächen aufweisen. Für die Prüfung des strukturellen Modells (Kap. 1.2.1) ist dieses System daher prinzipiell noch besser geeignet als $\mathrm{MgO}$, da hier die aus SANS- bestimmte Oberfläche der Teilchen direkt der Menge an $\{111\}$ - Grenzflächen entspricht. Die Bildungsenthalpie von $\mathrm{Mn}_{3} \mathrm{O}_{4}$ ist zudem geringer als die des $\mathrm{MgO}$. Aus diesem Grunde ist somit auch ein Vergleich der Bildungs- und Wachstumsmechanismen dieser beiden Oxide interessant.

\subsubsection{Innere Oxidation der AgMn- Legierung}

Im System Ag-0,4At.\%Mn wurden bei gleichen Oxidationstemperaturen größere Oxidteilchen erzeugt als im System Ag-0,4At.\%Mg. Dies ist begründet in der geringen Bildungsenthalpie des $\mathrm{Mn}_{3} \mathrm{O}_{4}$ und dem daraus resultierenden größeren kritischen Keimradius im Vergleich zu MgO. Bei einer Oxidationstemperatur von $750{ }^{\circ} \mathrm{C}$ bilden sich Ausscheidungen von etwa $5 \mathrm{~nm}$ Radius. Berücksichtigt man die geringe Detektionswahrscheinlichkeit des Sauerstoffs in TAP- Analysen, bestätigt eine Konzentrationsanalyse, daß es sich bei der ausgeschiedenen Phase um $\mathrm{Mn}_{3} \mathrm{O}_{4}$ handelt (Abb. 3.15).

Da bei einer Oxidationstemperatur von $750{ }^{\circ} \mathrm{C}$ die Teilchen so groß wurden, daß sie nur selten vollständig im Analysevolumen lagen, wurden die Proben bei einer Temperatur von $650{ }^{\circ} \mathrm{C}$ innenoxidiert. Aufgrund des kleineren kritischen Keimradius (siehe Kap. 1.1.2) und der langsameren Kinetik wurden bei dieser Temperatur wesentlich kleinere Ausscheidungen gebildet, die damit der Analyse mit TAP wieder zugänglich waren. Die zeitliche Entwicklung der gebildeten Oxidteilchen bei konstanter Oxidationstemperatur wurde dann analog zu Ag-0,4At.\%Mg untersucht. Die mit Hilfe von TAP bestimmten Werte sind in Tabelle 3.11 aufgeführt. 

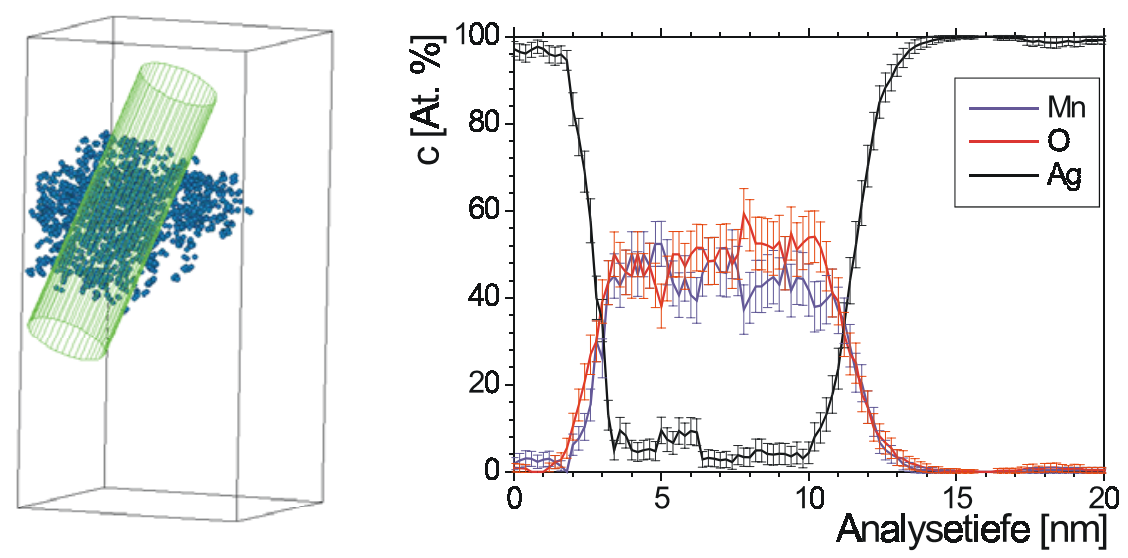

Abb. 3.15: Links: Angeschnittene $\mathrm{Mn}_{3} \mathrm{O}_{4^{-}}$Ausscheidung in $\mathrm{Ag}$, Maße der Box: $17 \times 17 \times 24$ nm; Rechts: Konzentrationsanalyse entlang des Analysezylinders im linken Bild. Der Analysezylinder liegt senkrecht zur vorderen und hinteren Grenzfläche.

Aus den Analysen ist es ebenfalls möglich, das Sauerstoff zu Mangan Verhältnis der für verschiedene Zeiten innenoxidierten Proben zu vergleichen. Hierbei muß allerdings berücksichtigt werden, daß nicht der gesamte Sauerstoff der Proben detektiert wird. Wie sich schon bei der Analyse der Ag/MgO- Proben zeigte, werden nur etwa 75-80 \% des Probensauerstoffs detektiert, da ein gewisser Teil des Probensauerstoffs zwischen den Hochspannungspulsen zusammen mit Wasserstoff des Restgases als HO- Komplex von der FIM- Spitze desorbiert. Dennoch ist ein systematischer Vergleich des Sauerstoffgehaltes unterschiedlicher Proben zulässig, da es sich um einen statistischen Effekt handelt. Die aus den Analysen bestimmten O/Mn- Verhältnisse finden sich ebenfalls in Tabelle 3.11.

Die mittlere Größe der $\mathrm{Mn}_{3} \mathrm{O}_{4}$ - Ausscheidungen nimmt wie im System $\mathrm{Ag} / \mathrm{MgO}$ mit zunehmender Oxidationszeit zu, während die Anzahldichte entsprechend abnimmt. Schon nach einer Oxidationsdauer von $\mathrm{t}_{\mathrm{IO}}=100 \mathrm{~min}$ haben sich Teilchen von etwa 0,9 nm Radius gebildet. Bis zu einer Auslagerungsdauer von 32 Stunden wird dann allerdings weder eine signifikante Größenzunahme der Ausscheidungen noch eine Abnahme in deren Anzahldichte $\mathrm{N}_{\mathrm{V}}$ beobachtet. Bei Oxidationszeiten länger als 32 Stunden werden dann wieder merklich größere Teilchen gebildet wobei die Anzahldichte stetig abnimmt. 
Tabelle 3.11: Aus TAP-Analysen bestimmte Werte von Radius R, Anzahldichte $N_{V}$ der $\mathrm{Mn}_{3} \mathrm{O}_{4^{-}}$Ausscheidungen, sowie die jeweiligen O/Mn-Verhältnisse innerhalb der Proben für verschiedene Oxidationsdauern $t_{I O}$, $\left(T_{I O}=650^{\circ} \mathrm{C}\right)$. Es sind die statistischen Fehler angegeben.

\begin{tabular}{l|cccccccc}
\hline$t_{I O}$ & $100 \min$ & $14 h$ & $24 h$ & $32 h$ & $76 h$ & $6 d$ & $14 d$ & $21 d$ \\
\hline \multirow{2}{*}{$\mathrm{R}[\mathrm{nm}]$} & 0,91 & 0,97 & 0,96 & 0,99 & 1,20 & 1,56 & 1,75 & 2,04 \\
& $\pm 0,07$ & $\pm 0,04$ & $\pm 0,05$ & $\pm 0,04$ & $\pm 0,05$ & $\pm 0,06$ & $\pm 0,06$ & $\pm 0,07$ \\
\multirow{2}{*}{$\mathrm{N}_{\mathrm{V}}\left[10^{24} \mathrm{~m}^{-3}\right]$} & 6,91 & 7,75 & 6,36 & 6,05 & 5,40 & 3,05 & 2,68 & 1,82 \\
& $\pm 0,82$ & $\pm 0,92$ & $\pm 1,22$ & $\pm 0,78$ & $\pm 0,12$ & $\pm 0,46$ & $\pm 0,43$ & $\pm 0,30$ \\
& 1,75 & 1,74 & 1,70 & 1,64 & 1,50 & 1,44 & 1,43 & 1,37 \\
$\mathrm{O} / \mathrm{Mn}$ & $\pm 0,04$ & $\pm 0,04$ & $\pm 0,04$ & $\pm 0,04$ & $\pm 0,05$ & $\pm 0,04$ & $\pm 0,04$ & $\pm 0,03$ \\
\hline
\end{tabular}

Für alle Oxidationszeiten wurde, trotz der möglicherweise geringeren Detektionswahrscheinlichkeit des $\mathrm{O}$, ein $\mathrm{O} / \mathrm{Mn}$ - Verhältnis oberhalb der 4:3- Stöchiometrie des $\mathrm{Mn}_{3} \mathrm{O}_{4}$ bestimmt (Tabelle 3.11). $\mathrm{Zu}$ Beginn der Oxidation beträgt das $\mathrm{O} / \mathrm{Mn}$ - Verhältnis sogar $\mathrm{O} / \mathrm{Mn}=1,75 \pm 0,04$. Nach $\mathrm{t}_{\mathrm{IO}}=21$ Tagen findet sich bei einer mittleren Teilchengröße von $\mathrm{R}=2,1 \mathrm{~nm}$ immerhin noch ein $\mathrm{O} / \mathrm{Mn}$ - Verhältnis von $1,37 \pm 0,03$. Eine chemische Analyse ${ }^{7}$ der Proben dieses Zustandes ergab ein O/Mn- Verhältnis von 1,66 $\pm 0,01$. Alle Proben hatten somit eine höhere O- Konzentration als nach der Stöchiometrie des Oxids zu erwarten wäre. Die erhöhte O- Konzentration folgt aus der Anwesenheit von Exzeß- $\mathrm{O}$ an der M/O- Teilchengrenzfläche, welche mit zunehmendem Teilchenradius kleiner wird.

\footnotetext{
${ }^{7}$ Mit Heißextraktion- Trägergas- Verfahren, G. Werner u. S. Hammoud, MPI Stuttgart.
} 


\subsection{SANS- Ergebnisse des Systems $\mathrm{Ag} / \mathrm{Mn}_{3} \mathrm{O}_{4}$}

Da die Gültigkeit des Kugel- Schale Modells in Kap. 3.2 schon für verschiedene Teilchenradien $\mathrm{R}$ und Kontraste $\Delta \rho_{\mathrm{S}}$ gezeigt werden konnte, kann nun auch überprüft werden, ob dieses Modell im Prinzip auch auf ein weiteres System angewendet werden kann. Im System $\mathrm{Ag} / \mathrm{Mn}_{3} \mathrm{O}_{4}$ ist im Unterschied $\mathrm{zu} \mathrm{Ag} / \mathrm{MgO}$ der Kontrast der Matrix größer als der des Oxids (siehe Kap. 2.2.1). Damit wird die Streulängendichte von Oxid zu Matrix für das System $\mathrm{Ag} / \mathrm{Mn}_{3} \mathrm{O}_{4}$ negativ:

$$
\Delta \rho_{\mathrm{Mn}_{3} \mathrm{O}_{4}-\mathrm{Ag}}=-1,95 \cdot 10^{10} \mathrm{~cm}^{-2} \quad \Delta \rho_{\mathrm{MgO}-\mathrm{Ag}}=+2,51 \cdot 10^{10} \mathrm{~cm}^{-2}
$$

Der Schalenkontrast $\left(\Delta \rho_{\mathrm{S}} \delta \mathrm{R}\right)_{\mathrm{H}}$ von Wasserstoff bleibt hingegen negativ und der von Deuterium $\left(\Delta \rho_{S} \delta R\right)_{D}$ weiterhin positiv. Nach Gl. (3.4) wird somit bei negativem $\Delta \rho_{K M}$ der Nettostreuquerschnitt $\mathrm{d} \Sigma /\left.\mathrm{d} \Omega\right|_{\mathrm{S}}{ }^{0}$ für Wasserstoff positiv und für Deuterium negativ.

$$
\left.\frac{\mathrm{d} \Sigma}{\mathrm{d} \Omega}\right|_{\text {Schale }} ^{0}=\frac{\left.\frac{\mathrm{d} \Sigma}{\mathrm{d} \Omega}\right|_{\mathrm{K}+\mathrm{L}}-\left.\frac{\mathrm{d} \Sigma}{\mathrm{d} \Omega}\right|_{\mathrm{K}}}{\left.\frac{\mathrm{d} \Sigma}{\mathrm{d} \Omega}\right|_{\mathrm{K}}}=\frac{6 \Delta \rho_{\mathrm{S}} \delta \mathrm{R}}{\Delta \rho_{\mathrm{KM}} \mathrm{R}} \cdot \exp \left(-\frac{\mathrm{Q}^{2} \mathrm{R}^{2}}{15}\right) .
$$

Beim System $\mathrm{Ag} / \mathrm{MgO}$ ist $\Delta \rho_{\mathrm{KM}}$ positiv und daher liegt die Streukurve der $\mathrm{H}$ - beladenen Probe unterhalb der Streukurve der D- beladenen Probe. Für das System $\mathrm{Ag} / \mathrm{Mn}_{3} \mathrm{O}_{4}$ ist hingegen zu erwarten, daß sich die Streukurve der H- beladenen Probe oberhalb der Streukurve der D- beladenen Kurve befindet. Nach der Variation von Teilchenradius R und Schalenkontrast $\Delta \rho_{S}$ kann überprüft werden, ob das Kugel- Schale Modell auch bei Variation der Streulängendichtedifferenz $\Delta \rho_{\mathrm{KM}}$ seine Gültigkeit behält. Ein weiterer Unterschied des Systems $\mathrm{Ag} / \mathrm{M}_{3} \mathrm{O}_{4} \mathrm{zu} \mathrm{Ag} / \mathrm{MgO}$ besteht in der Morphologie der gebildeten Ausscheidungen. Für $\mathrm{Mn}_{3} \mathrm{O}_{4}$ - Teilchen entspricht die aus SANS ermittelte H- Belegungsdichte der H- Belegungsdichte der $\{111\}$ - Flächen, da die Ausscheidungen allein aus diesen Flächen bestehen (vgl. Kap. 1.2.1). 
Abb. 3.16 zeigt das Streuverhalten einer H- und D- beladenen $\mathrm{Ag} / \mathrm{Mn}_{3} \mathrm{O}_{4^{-}}$Probe im Vergleich zu dem Streuverhalten einer unbeladenen Probe. Wie nach Gleichung (3.4) zu erwarten, liegt die Streukurve der H- beladenen Probe oberhalb der Streukurve der Dbeladenen Probe. Allerdings befindet sich die Streukurve der unbeladenen $\mathrm{Ag} / \mathrm{Mn}_{3} \mathrm{O}_{4}$ Probe ebenfalls unterhalb der D- beladenen Kurve. Da das für diese Messungen verwendete SANS- Instrument nicht die Möglichkeit bot die Proben während des Experiments zu evakuieren, weisen alle drei Streukurven, als Folge einer zusätzlichen Luftstreuung, einen sehr hohen Untergrund von $\sim 0,026 \mathrm{~cm}^{-1}$ auf. Aufgrund der geringen Intensität der Streukurve der unbeladenen Probe, ist diese für Werte $Q>0,08$ $\AA^{-1}$ durch den hohen Untergrund bestimmt.

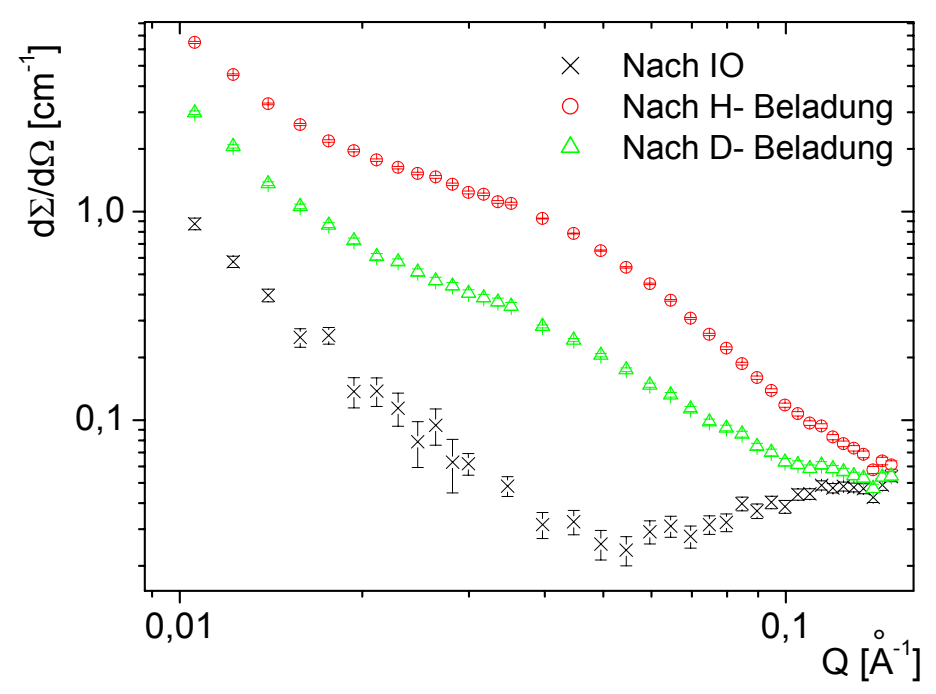

Abb. 3.16: Makroskopischer Streuquerschnitt $d \Sigma / d \Omega$ von innenoxidierten Ag1At.\%Mn-Proben vor und nach H/D-Beladung, Teilchenradius $R=2,9 \mathrm{~nm}$. Der Guinier-Bereich liegt bei $Q \sim 0,4$ A.

Aufgrund der geringen, gestreuten Intensität $\mathrm{d} \Sigma / \mathrm{d} \Omega$ der unbeladenen Probe und der daraus resultierenden, schlechten Meßstatistik wurde die $\mathrm{H}$ - Belegungsdichte der $\mathrm{Ag} / \mathrm{Mn}_{3} \mathrm{O}_{4}$ - Proben aus dem unnormierten Nettostreuquerschnitt $\mathrm{d} \Sigma /\left.\mathrm{d} \Omega\right|_{\mathrm{B}-\mathrm{UB}}$ ermittelt (Gleichung (3.5)). Die Ableitung dieses unnormierten Nettostreuquerschnitts findet sich im Appendix in Kap. 6.1. 


$$
\left.\frac{\mathrm{d} \Sigma}{\mathrm{d} \Omega}\right|_{\mathrm{B}-\mathrm{UB}}=\left.\frac{\mathrm{d} \Sigma}{\mathrm{d} \Omega}\right|_{\mathrm{B}}-\left.\frac{\mathrm{d} \Sigma}{\mathrm{d} \Omega}\right|_{\mathrm{UB}} \cong 8 \pi \cdot \mathrm{v} \cdot\left(\Delta \rho_{\mathrm{S}} \delta \mathrm{R}\right) \cdot \Delta \rho_{\mathrm{Mn}_{3} \mathrm{O}_{4}-\mathrm{Ag}} \cdot \exp \left(-\frac{4 \mathrm{Q}^{2} \mathrm{R}^{2}}{15}\right) .
$$

$\mathrm{d} \Sigma /\left.\mathrm{d} \Omega\right|_{\mathrm{B}}$ und $\mathrm{d} \Sigma /\left.\mathrm{d} \Omega\right|_{\text {UB }}$ sind dabei die makroskopischen Streuquerschnitte einer H- (oder D-) beladenen Probe und der unbeladenen Probe. Der Teilchenradius R = 2,9 nm wurde aus Kleinwinkelröntgenstreuungs (SAXS)- Messungen ermittelt [KLUTHE03C]. Der in (3.5) benötigte Volumenbruchteil $v$ des Oxids konnte mit Hilfe einer Mikrosondenanalyse bestimmt werden (siehe Kap. 2.3.3). Der Vorteil von einer Auswertung über Gl. (3.5) liegt darin, daß den Guinier- Bereich beeinflussende andere Streuungen mit abgezogen werden. Durch eine Differenzbildung der H- und D- beladenen Streukurve erhält man aus Gleichung (3.5):

$$
\left.\frac{\mathrm{d} \Sigma}{\mathrm{d} \Omega}\right|_{\mathrm{H}-\mathrm{D}}=\left.\frac{\mathrm{d} \Sigma}{\mathrm{d} \Omega}\right|_{\mathrm{H}}-\left.\frac{\mathrm{d} \Sigma}{\mathrm{d} \Omega}\right|_{\mathrm{D}} \cong 8 \pi \cdot \mathrm{v} \cdot \mathrm{R}^{2} \cdot \Theta_{\mathrm{H}}\left(\mathrm{b}_{\mathrm{H}}-\mathrm{b}_{\mathrm{D}}\right) \cdot \Delta \rho_{\mathrm{Mn}_{3} \mathrm{O}_{4}-\mathrm{Ag}} \cdot \exp \left(-\frac{4 \mathrm{Q}^{2} \mathrm{R}^{2}}{15}\right)
$$

$\mathrm{d} \Sigma /\left.\mathrm{d} \Omega\right|_{\mathrm{H}-\mathrm{D}}$ ist in G1. (3.6) der Nettostreuquerschnitt der segregierten Schale und $\mathrm{d} \Sigma /\left.\mathrm{d} \Omega\right|_{\mathrm{H}}, \mathrm{d} \Sigma /\left.\mathrm{d} \Omega\right|_{\mathrm{D}}$ sind die makroskopischen Streuquerschnitte der H- und D- beladenen Probe. Abb. 3.17a zeigt eine Auftragung von $\ln \mathrm{d} \Sigma /\left.\mathrm{d} \Omega\right|_{\mathrm{H}-\mathrm{D}}$ gegen $(\mathrm{QR})^{2}$. Die H- Belegungsdichte $\mathrm{Ag} / \mathrm{Mn}_{3} \mathrm{O}_{4}$ - Teilchengrenzfläche konnte mit diesen Werten dann aus deren Achsenabschnitt ermittelt werden:

$\Theta_{\mathrm{H}}=(1,5 \pm 0,2) \cdot 10^{15} \mathrm{~cm}^{-2}$.

Dieser Wert $\Theta_{\mathrm{H}}$ ist etwa doppelt so groß wie der für das System $\mathrm{Ag} / \mathrm{MgO}$. Der Schalenkontrast für Wasserstoff und Deuterium kann nach Formel (3.5) aus einer Auftragung von $\mathrm{d} \Sigma /\left.\mathrm{d} \Omega\right|_{\mathrm{B}-\mathrm{UB}}$ gegen $(\mathrm{QR})^{2}$ aus dem Achsenabschnitt einer Anpassungsgerade im Guinier- Bereich bestimmt werden (Abb. 3.17b). Die Schalenkontraste betragen: $\left(\Delta \rho_{\mathrm{S}} \delta \mathrm{R}\right)_{\mathrm{H}}=2446 \mathrm{~cm}^{-1}$ und $\left(\Delta \rho_{\mathrm{S}} \delta \mathrm{R}\right)_{\mathrm{D}}=673 \mathrm{~cm}^{-1}$. Mit Kenntnis der Schalenkontraste sowie $\Theta_{\mathrm{H}}$, läßt sich nun auch die Änderung der Ag- Matrixdichte nach (2.19) bestimmen. Es berechnet sich eine Ag- Dichteänderung von

$$
\Delta \Theta_{\mathrm{Ag}}=(2,7 \pm 0,5) \cdot 10^{15} \mathrm{~cm}^{-2}
$$


Die Ag- Dichteänderung $\Delta \Theta_{\mathrm{Ag}}$ ist somit etwa doppelt so groß wie die H- Belegungsdichte $\Theta_{\mathrm{H}}$. Der Wert für $\Delta \Theta_{\mathrm{Ag}}$ hat allerdings einen recht großen Fehler, da die Streukurve der unbeladenen Probe mit großen statistischen Fehlern behaftet ist.
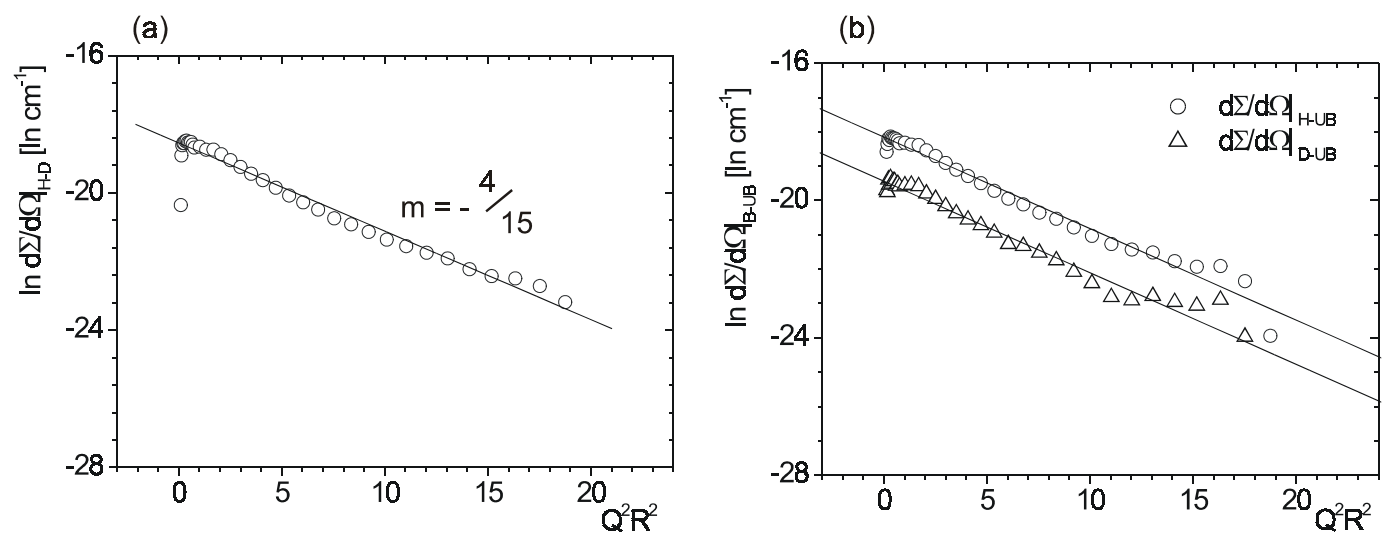

Abb. 3.17: Guinier- Auftragungen der Nettostreuquerschnitte einer innenoxidierten Ag-1At.\%Mn-Probe; $R=2,9 \mathrm{~nm}$. (a) $d \Sigma /\left.d \Omega\right|_{H-D}$ gegen $Q^{2} R^{2}$ nach Gl. (3.6); (b) $d \Sigma /\left.d \Omega\right|_{B-U B}$ nach Gl. (3.5). 


\section{Diskussion}

\subsection{Bildungskinetik und Reifung der Oxidteilchen}

\subsubsection{Frühstadien}

In Kap. 3.1.1 konnte gezeigt werden, daß die O- Konzentration schon nach einer Oxidationszeit von $t_{I O}=10 \mathrm{~min}$ der Mg-Konzentration entspricht. Die Oxidationsfront (siehe Kap. 1.1.1) hat die Probe nach dieser Zeit also schon vollständig durchwandert. Alle TAP- Massenspektren weisen zudem, als Folge einer nicht homogenen Feldverdampfung des $\mathrm{MgO}$, nicht nur $\mathrm{Mg}^{+}$- und $\mathrm{O}^{+}$- Ionen, sondern auch $\mathrm{MgO}^{+}$- Molekülionen auf. Die Detektion eines $\mathrm{MgO}^{+}$- Ions ist nur dann möglich, wenn zwei in der Probe direkt benachbarte $\mathrm{Mg}$ - und O- Atome gleichzeitig feldverdampfen (siehe Abb. 4.1).
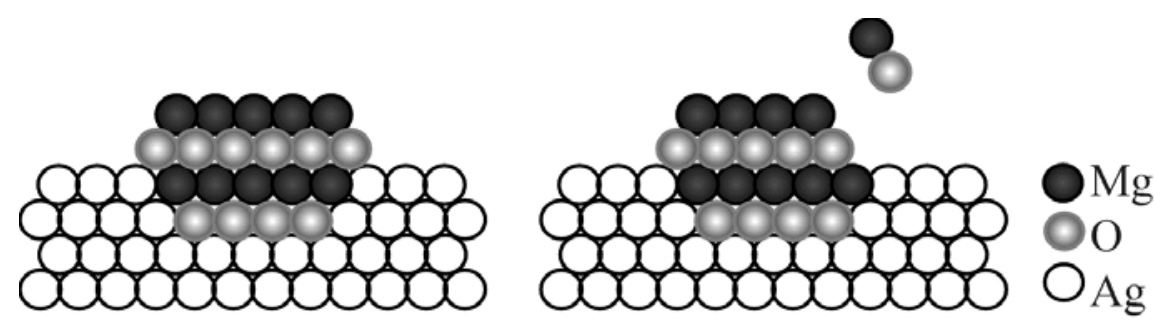

Abb. 4.1: Schematische Skizze zum Feldverdampfen eines $\mathrm{MgO}$-Moleküls.

Da die als $\mathrm{MgO}^{+}$detektierten $\mathrm{Mg}$ - Atome für alle Oxidationszeiten einen Anteil von 10$15 \%$ aller $\mathrm{Mg}$ - Atomen einnehmen, bedeutet dies, daß schon nach $\mathrm{t}_{\mathrm{IO}}=10 \mathrm{~min}$ alle $\mathrm{Mg}$ Atome durch O- Atome gebunden sind. Einem $\chi^{2}$ - Test zufolge, liegen sowohl O, als auch Mg in inhomogener Verteilung innerhalb der Probe vor (siehe Tabelle 3.2). Nach einer Oxidationszeit von $10 \mathrm{~min}$ haben sich schon Oxidteilchen von $\mathrm{R} \approx 0,6 \mathrm{~nm}$ gebildet. Nach Wagner hat die Oxidationsfront die Probe allerdings schon nach $\mathrm{t} \approx 30$ Sekunden durchlaufen (siehe Kap. 1.1.1). Für den Zustand des Systems unmittelbar nach Durchschreiten der Oxidationsfront sind zwei unterschiedliche Entwicklungen denkbar: 
(i) Bei Vernachlässigung der Diffusion der $\mathrm{Mg}$ - Atome werden diese alle durch $\mathrm{O}$ gebunden, bevor sich freie $\mathrm{Mg}$ - Atome an ein gebildetes $\mathrm{MgO}$ - Molekül anlagern können. In diesem idealisierten Fall liegt damit nach dem Durchschreiten der Oxidationsfront eine homogenen Verteilung von $\mathrm{MgO}$ - Molekülen innerhalb des übersättigten Ag- Mischkristalls vor [RIKEL99]. Das System entmischt sich dann über eine Diffusion dieser MgO- Moleküle (siehe Kap. 4.1.2) in kugelförmige Ausscheidungen, analog zur Phasenseparation metallischer Systeme.

(ii) Für den Fall einer nicht zu vernachlässigenden Diffusion der Mg- Atome ist keine homogene Verteilung an MgO- Molekülen nach Durchlaufen der Oxidationsfront zu erwarten. Angesichts der hohen Sauerstoffaffinität des $\mathrm{Mg}$ können sich freie MgAtome an ein gebildetes $\mathrm{MgO}-$ Molekül anlagern. In diesem Fall sind auch regellose, unsymmetrische $\mathrm{MgO}$ - Cluster aus mehreren Atomen denkbar [CHARRIN00], da anzunehmen ist, daß die Enthalpie der chemischen Bindung groß gegen die aufzubringende Grenzflächenenergie ist. Im Verlauf einer weiteren Glühung werden aus diesen unsymmetrischen Clustern dann kohärente Ausscheidungen. In diesem Fall sollten die in den Frühstadien gebildeten Ausscheidungen weniger kompakt sein, als zu späteren Stadien.

Allein aus den TAP- Untersuchungen laßt sich nicht ableiten, ob die Ausscheidungen früherer Stadien kompakter sind, als die späterer Stadien, da die Rekonstruktion der Ausscheidungen durch ein Abbildungsartefakt verfälscht wird. Die SANS- Ergebnisse zeigen allerdings, daß die Proben der frühen Stadien $\left(t_{I O} \leq 1 d\right)$ einen höheren ausgeschiedenen Volumenbruchteil von $v \approx 0,8$ Vol.\% aufweisen, als länger oxidierter Proben mit $v \approx 0,7$ Vol.\%. Diese Beobachtung steht im Einklang mit dem Reaktionsablauf (ii), da bei gleicher MgO- Konzentration regellose Ausscheidungen einen größeren Volumenbruchteil einnehmen, als kugelförmige Ausscheidungen. Es ist ebenfalls denkbar, daß dieser größere Volumenbruchteil aus höheren O- Konzentration innerhalb der Keimbildungsphase resultiert. 

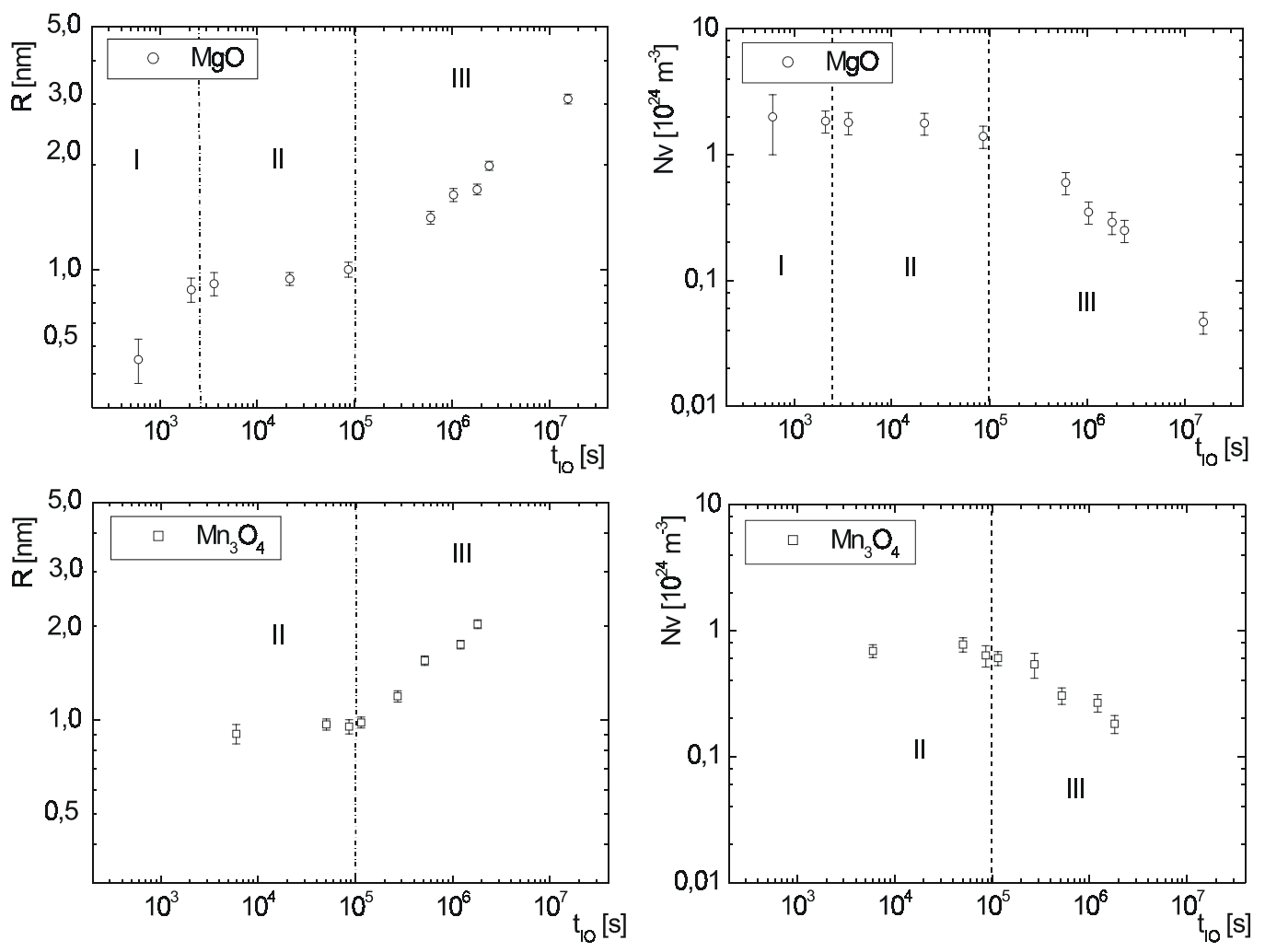

Abb. 4.2: Doppeltlogarithmische Auftragungen der Teilchenradien und Anzahldichten gegen die Oxidationsdauer für $\mathrm{MgO}$ und $\mathrm{Mn}_{3} \mathrm{O}_{4}$ in $\mathrm{Ag}$. Bereich I: Keimbildung, Bereich II: Übergangsbereich, Bereich III: Vergröberung.

Für beide untersuchten Systeme weist der Verlauf der Teilchengröße mit zunehmender Oxidationsdauer ein Plateau auf (siehe Abb. 4.2, Bereich II). Die Teilchen werden nicht stetig größer, sondern besitzen bis zu einer Auslagerungsdauer von etwa 24 Stunden den gleichen mittleren Radius von $\mathrm{R} \approx 0,9 \mathrm{~nm}$. Die Anzahldichte der Ausscheidungen ist innerhalb dieser Zeitspanne ebenfalls konstant. Im Rahmen der klassischen Keimbildungstheorie ist ein solches Plateau der Entwicklung von Teilchenradius und Anzahldichte nicht vorgesehen. Tatsächlich wird ein solches Verhalten allerdings auch bei der Phasenseparation metallischer Systeme beobachtet [WAGNER91]. Kampmann und Wagner interpretieren dieses Plateau als Übergangsbereich von Keimbildung zu Umlösung [WAGNER91]. Für innenoxidierte Ag-0,4At.\%Mg- Legierung deuten die SANSErgebnisse dieser Arbeit auf eine Änderung der Teilchenmorphologie innerhalb dieses Bereichs II hin. Wie bereits erwähnt, nehmen die Oxidausscheidungen der Frühstadien einen größeren Volumenbruchteil ein, als die Ausscheidungen späterer Stadien. Diese 
Beobachtung steht im Einklang mit Charrin et. al., wonach sich in den Frühstadien der Inneren Oxidation regellose, nicht notwendigerweise stöchiometrische $\mathrm{MgO}$ - Cluster bilden [CHARRIN00]. Für den Fall, daß diese Cluster einen Sauerstoffüberschuß aufweisen, stellen diese damit auch einen höheren Volumenbruchteil dar, als die kohärenten und stöchiometrischen Ausscheidungen späterer Stadien. Ein weiteres Indiz für eine Veränderung der Teilchenmorphologie innerhalb des Bereichs II liefern die HBeladungsexperimente. Es konnte gezeigt werden, daß die Grenzflächen der Oxidteilchen, die zu Beginn des Plateaus mit $\mathrm{H}$ beladen wurden eine kleinere H-Belegungsdichte $\left(\Theta_{\mathrm{H}}=2,2 \cdot 10^{14} \mathrm{~cm}^{-2}\right)$ aufwiesen, als die länger oxidierten am Ende des Plateaus $\left(\Theta_{\mathrm{H}}=5,7 \cdot 10^{14} \mathrm{~cm}^{-2}\right)$. Die unterschiedlichen Belegungsdichten sind ein eindeutiges Maß für die unterschiedlichen Grenzflächenbeschaffenheiten der entsprechenden Oxidteilchen, was in Kap. 4.2.4 gezeigt wird. Es ist daher denkbar, daß sich mit dem Durchschreiten der Oxidationsfront regellose MgO- Ausscheidungen bilden, welche dann im Verlauf einer weiteren Glühung zu kohärenten Ausscheidungen kompaktieren. Diese Interpretation ist physikalisch sinnvoll, da bereits Combe und Charrin zeigten, daß $\mathrm{MgO}$ - Oxidkeime regelloser Form eine sehr starke Verzerrung der Matrix bewirken. Um diese Verzerrungsenergie zu minimieren ist es daher notwendig, die Oberfläche durch Umlagerungsprozesse der $\mathrm{MgO}$ - Moleküle möglichst glatt zu gestalten. Die verbleibenden Verzerrungsfelder werden dann durch den Einbau von Versetzungen in der Matrix abgebaut [CHARRIN93, CHARRIN98]. Eine weitere treibende Kraft besteht in der Minimierung der totalen M/O- Grenzfläche durch die Kompaktierung der Oxidteilchen.

\subsubsection{Spätstadien}

Im Anschluß an Bereich II zeigen sowohl MgO- als auch $\mathrm{Mn}_{3} \mathrm{O}_{4}$ - Ausscheidungen mit zunehmender Oxidationsdauer ein deutliches Reifungsverhalten. Die Tatsache, daß bei oxidischen Ausscheidungen mit derart hohen Bildungsenthalpien (vgl. Kap. 1.1.2) eine Umlösung stattfindet, ist nicht selbstverständlich. Diverse Autoren gingen daher bisher von einem sehr geringen [CHARRIN98], bis zu vernachlässigenden Reifungsverhalten [GEGNER95, ORTH96] von MgO- Ausscheidungen in einer metallischen Matrix aus. 
Die treibende Kraft für die Umlösung der Oxidausscheidungen ist die Minimierung der gesamten Grenzflächenenergie. Nach der bisherigen Meinung ist die Voraussetzung für eine solche Umlösung, daß die Ausscheidungen durch die Absorbtion (bzw. Emission) einzelner Atome wachsen (bzw. schrumpfen) [WAGNER61]. Aufgrund der hohen Sauerstoffdiffusion sollte nach dieser Vorstellung die Diffusion von Mg (bzw. Mn)- Atomen der bestimmende Faktor für eine Reifung der Oxidteilchen sein. Es sollten daher zwischen den Oxidteilchen einzelne $\mathrm{Mg}$ - Atome zu finden sein. Die TAP- Ergebnisse zeigen jedoch, daß auch der Anteil des $\mathrm{Mg}$, der nicht in Form größerer Oxidteilchen agglomeriert ist, durch O- Atome gebunden ist. Es befinden sich also keine freien $\mathrm{Mg}$ Atome in der Probe, was angesichts der großen Affinität zwischen $\mathrm{Mg}$ und $\mathrm{O}$ auch zu erwarten ist. Offensichtlich kann eine Reifung der MgO- Teilchen nicht wie die klassische Ostwaldreifung, mittels atomarer Umlösungsprozesse vonstatten gehen. Im Rahmen dieser Arbeit wird deshalb vorgeschlagen, daß sich die Reifung der MgOAusscheidungen durch eine Diffusion von $\mathrm{MgO}_{\mathrm{x} \geq 1}$ - Einheiten vollzieht (vgl. Abb. 4.3b).
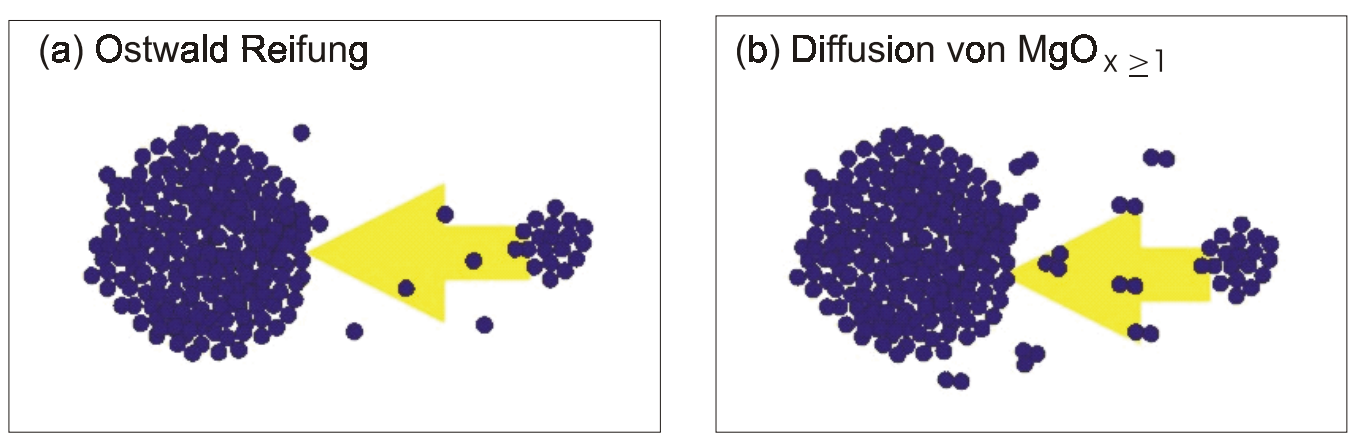

Abb. 4.3: Skizzen zur (a) Ostwaldreifung und (b) der im Rahmen dieser Arbeit vorgeschlagenen Reifung der Oxidteilchen über eine Diffusion von $\mathrm{MgO}_{x \geq 1}$.

Das Atomvolumen von Silber beträgt $\Omega_{\mathrm{Ag}}=17,06 \AA^{3}$, das Volumen eines MgOMoleküls $\Omega_{\mathrm{MgO}}=18,69 \AA^{3}$. Zwar dehnt aufgrund seines größeren Volumens das MgOMolekül das Matrixgitter leicht auf, jedoch ist die Volumendifferenz zwischen $\mathrm{MgO}$ und Ag mit weniger als $10 \%$ immer noch klein genug, um $\mathrm{MgO}$ eine nicht zu vernachlässigende Beweglichkeit in $\mathrm{Ag} \mathrm{zu}$ verleihen. Der für die Umlösung notwendige Materiestrom kann also durch die Diffusion von $\mathrm{MgO}_{\mathrm{x} \geq 1}$ - Komplexen erbracht werden. Die Diffusionsbewegung einer solchen $\mathrm{MgO}_{\mathrm{x} \geq 1}$ - Spezies ist nach Charrin et. al. durch ein gegenseitiges „Hangeln“ von $\mathrm{Mg}$ - und O- Atomen durch das Matrixgitter sogar möglich, ohne daß die Mg-O- Bindung zerstört wird [CHARRIN92]. Dabei diffundieren 
die $\mathrm{MgO}_{\mathrm{X} \geq 1-}$ - Moleküle interstitiell - korreliert durch das Volumen, wobei ein Atom substitutionell diffundiert und das zweite Atom mit diesem im Zwischengitter mitwandert [MURCH91]

Im Falle der $\mathrm{Mn}_{3} \mathrm{O}_{4}$ - Ausscheidungen ergibt sich durch die geringere Bildungsenthalpie dieses Oxids ein größerer kritischer Keimradius als für $\mathrm{MgO}$. Die Reifung dieser Teilchen könnte daher durchaus wie eine klassische Ostwaldreifung ablaufen, da eine Dissoziation des Oxids in freie Mn- und O- Atome möglich ist. In den Massenspektren der innerlich oxidierten Ag-0,4At.\%Mn- Proben traten allerdings $\mathrm{MnO}^{+}, \mathrm{MnO}_{2}{ }^{+}, \mathrm{MnO}_{3}{ }^{+}$ und sogar $\mathrm{MnO}_{4}{ }^{+}$- Ionen auf. Diese $\mathrm{MnO}_{\mathrm{x} \geq 1^{-}}$Molekülionen entsprechen nicht der chemisch zu erwartenden Stöchiometrie und wurden bevorzugt im Bereich zwischen den $\mathrm{Mn}_{3} \mathrm{O}_{4}$ - Ausscheidungen detektiert. Der kritische Keimradius von $\mathrm{MnO}$ in Ag liegt bei $650{ }^{\circ} \mathrm{C}$ mit $1,65 \AA$ unterhalb des Molekülradius des $\mathrm{MnO}$ von $1,75 \AA$. Es ist also auch in diesem System möglich, daß die $\mathrm{Mn}_{3} \mathrm{O}_{4}$ - Teilchen nicht in einzelne Atome dissozieren, sondern auch in $\mathrm{MnO}_{\mathrm{x} \geq 1}$ - Einheiten. Dies legt den Schluß nahe, daß auch in diesem System eine Vergröberung über die Diffusion von $\mathrm{MnO}_{\mathrm{x} \geq 1}$ - Spezies analog zum System $\mathrm{Ag} / \mathrm{MgO}$ stattfindet.

\section{Ratenkonstante und Zeitexponent der Umlösung}

Nach der LSW- Theorie [Lifshitz61, WAGNER61] gilt für den Fall eines diffusionsbestimmten Wachstums ein sogenanntes $t^{1 / 3}$ - Gesetz für die Zunahme des mittleren Teilchenradius mit der Zeit:

$$
\mathrm{R}^{3}(\mathrm{t})-\mathrm{R}_{0}^{3}=\alpha_{\mathrm{LSW}} \cdot \mathrm{t}, \text { wobei } \quad \alpha_{\mathrm{LSW}}=\frac{8 \cdot \sigma \cdot \mathrm{D} \cdot \mathrm{c}_{\mathrm{e}}^{\mathrm{v}} \cdot \mathrm{V}_{\mathrm{m}}^{2}}{9 \cdot \mathrm{R} \cdot \mathrm{T} \cdot \beta^{2}}
$$

$\mathrm{R}_{0}$ ist der Radius zu Beginn der Reifung, $\beta$ der Stöchiometriekoeffizient des Oxids, $\sigma$ die Grenzflächenenergie, D der Diffusionskoeffizient der diffundierenden Spezies und $\mathrm{c}_{\mathrm{e}}{ }^{\mathrm{v}}$ deren Restlöslichkeit in der Matrix im thermodynamischen Gleichgewicht (in $\left.\mathrm{mol} / \mathrm{cm}^{3}\right) . \mathrm{V}_{\mathrm{m}}$ ist das Molvolumen der ausgeschiedenen Phase. Die LSW- Theorie geht von einer Umlösung durch atomare Diffusion aus. Für $\mathrm{Ag} / \mathrm{MgO}$ ist diese Voraussetzung nicht erfüllt, weil die Reifung über eine Diffusion von $\mathrm{MgO}_{\mathrm{x} \geq 1}$ - Einheiten abläuft. Aller- 
dings ist der Umlösungsvorgang auch für dieses System diffusionsgesteuert, womit ein $\mathrm{t}^{1 / 3}$ - Gesetz nach (4.1) für die Entwicklung des Teilchenradius mit zunehmender Oxidationsdauer zu erwarten wäre. Aus einer Auftragung $\log \left(\mathrm{R}^{3}-\mathrm{R}_{0}^{3}\right)^{1 / 3}=\mathrm{n} \cdot \log \mathrm{t}+$ $\log \alpha_{\mathrm{LSW}}{ }^{1 / 3}$ ist es möglich (siehe Abb. 4.4), für beide Systeme die Ratenkonstante $\alpha_{\text {LSW }}$ sowie den Zeitexponenten der Umlösung zu bestimmen. Aus dieser kann dann nach (4.1) das Produkt $\sigma \cdot \mathrm{D} \cdot \mathrm{c}_{\mathrm{e}}{ }^{\mathrm{v}}$ berechnet werden (Tabelle 4.1).

Tabelle 4.1: Berechnete Ratenkonstanten $\alpha_{L S W}$ und Produkte $\sigma \cdot D \cdot c_{e}^{v}$ der Umlösung für $\mathrm{MgO}$ und $\mathrm{Mn}_{3} \mathrm{O}$ in $\mathrm{Ag}$.

\begin{tabular}{c|ccc}
\hline Oxid & $\mathrm{n}$ & $\alpha_{\text {LSW }}\left[\mathrm{cm}^{3} / \mathrm{s}\right]$ & $\sigma \cdot \mathrm{D} \cdot \mathrm{c}_{\mathrm{e}}{ }^{\mathrm{v}}\left[\mathrm{Jmol} \cdot \mathrm{cm}^{-3} \mathrm{~s}^{-1}\right]$ \\
\hline $\mathrm{MgO}$ & $0,27 \pm 0,01$ & $(4,8 \pm 0,2) \cdot 10^{-26}$ & $3,63 \cdot 10^{-24}$ \\
$\mathrm{Mn}_{3} \mathrm{O}_{4}$ & $0,33 \pm 0,02$ & $(4,0 \pm 0,4) \cdot 10^{-26}$ & $2,70 \cdot 10^{-25}$ \\
\hline
\end{tabular}

(a)

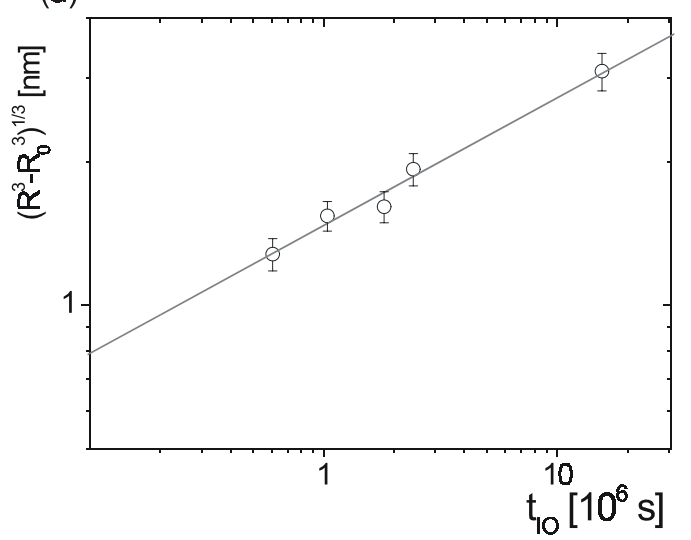

(b)

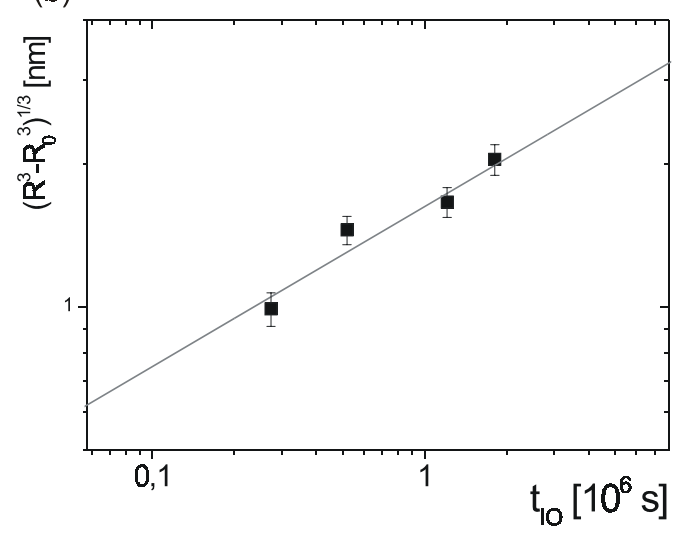

Abb. 4.4: Auftragungen $\log \left(R^{3}-R_{0}^{3}\right)^{1 / 3}$ gegen die Oxidationsdauer to für Oxidteilchen im Bereich der Umlösung; (a) $\mathrm{Ag} / \mathrm{MgO}$ und (b) $\mathrm{Ag} / \mathrm{Mn}_{3} \mathrm{O}_{4}$.

Aus der Ratenkonstante kann mit Kenntnis der Restlöslichkeiten, sowie der Grenzflächenenergie der Diffusionskoeffizient der diffundierenden Spezies berechnet werden. Für das System MgO betrug $\mathrm{c}_{\mathrm{e}}^{\mathrm{v}}=2,8 \cdot 10^{-4}=2,5 \cdot 10^{-5} \mathrm{~mol} / \mathrm{cm}^{3}$. Als Wert für die Grenzflächenenergie wurde $\sigma_{\mathrm{Ag} / \mathrm{MgO}}=2 \cdot 10^{-4} \mathrm{~J} / \mathrm{cm}^{2}$ aus [MEIJERING71] verwendet. Damit ergibt sich bei einer Temperatur von $\mathrm{T}=750^{\circ} \mathrm{C}$ ein Diffusionskoeffizient der $\mathrm{MgO}_{\mathrm{x} \geq 1^{-}}$ Moleküle in Ag von 
$\mathrm{D}_{\mathrm{MgO}_{\mathrm{X} \geq 1}}\left(750^{\circ} \mathrm{C}\right) \approx 7 \cdot 10^{-16} \mathrm{~cm}^{2} / \mathrm{s}$

Dieser Diffusionskoeffizient ist etwa 6 Größenordnungen kleiner als der des $\mathrm{Mg}$ in $\mathrm{Ag}$ Matrix. Durch die sehr viel höhere Matrixkonzentration der $\mathrm{MgO}_{\mathrm{x} \geq 1^{-}}$Moleküle im Vergleich zu freien Mg-Atomen, ist diese Beweglichkeit allerdings groß genug um die beobachtete Reifung der MgO- Ausscheidungen zu erklären.

Das System $\mathrm{Ag} / \mathrm{MgO}$ weist außerdem eine Abweichung vom dem zu erwartenden Reifungsexponenten von 1/3 auf (Tabelle 4.1). Nach G1. (4.1) kann eine solche Abweichung dadurch erklärt werden, daß die Konstante $\alpha_{\text {LSw }}$ eine Zeitabhängigkeit aufweist, also $\alpha_{\mathrm{LSW}}=\alpha_{\mathrm{LSW}}(\mathrm{t})$. Dies bedeutet, daß entweder die Grenzflächenenergie $\sigma$, der Diffusionskoeffizient D oder $\mathrm{c}_{\mathrm{e}}{ }^{\mathrm{v}}$ mit zunehmender Oxidationsdauer abnehmen. Pedder et. Al. fanden bei der Untersuchung des Umlösungsverhaltens von CdO- Ausscheidungen in Ag einen Reifungsexponent der ebenfalls kleiner 1/3 war [PEDDER78]. Die Gemeinsamkeit der Systeme $\mathrm{Ag} / \mathrm{CdO}$ und $\mathrm{Ag} / \mathrm{MgO}$ besteht darin, daß sowohl $\mathrm{CdO}-$ als auch MgO- Ausscheidungen, in Ag die Form abgestumpfter Oktaeder aus $\{100\}-$ und $\{111\}$ - Facetten aufweisen. Die SANS- Ergebnisse dieser Arbeit zeigen nun, daß der Anteil der $\{111\}$ - Grenzflächen mit zunehmender Teilchengröße auf Kosten der $\{100\}$ Grenzflächen ansteigt (siehe Kap. 4.2.4). Da die $\{111\}$ - Grenzflächen eine geringere Grenzflächenenergie besitzen, als die $\{100\}$ - Grenzflächen, bedeutet dieses Ergebnis, daß die mittlere Grenzflächenenergie $\sigma_{\mathrm{Ag} / \mathrm{MgO}}$ mit zunehmender Teilchengröße abnimmt. Mit steigender Glühdauer wird daher die treibende Kraft für die Vergröberung geringer. Die geringe Reifungsneigung des $\mathrm{MgO}$ ist somit auch durch eine Abnahme der Grenzflächenenergie mit zunehmender Glühdauer zu verstehen.

Des weiteren zeigte sich, daß Proben ohne Exzeß- O nach gleicher Glühdauer einen größeren, mittleren Teilchenradius aufweisen als Proben mit Exzeß- O (Tabelle 3.3). Dieser Effekt kann auf Basis des strukturellen Modells von Huang, Mader und Kirchheim verstanden werden, wonach der Exzeß Sauerstoff nur an der $\{111\}$ - Grenzfläche, aber nicht an der $\{100\}$ - Grenzfläche segregieren kann [HUANG91]. Durch eine Entfernung des Exzeß- Sauerstoffs aus der Probe wird somit die Grenzflächenenergie der 
$\{111\}$ - Facetten erhöht. Dies zeigt sich beispielsweise daran, daß selbst $\mathrm{Mn}_{3} \mathrm{O}_{4}$ Ausscheidungen nach einer Hochtemperaturglühung im UHV, nicht mehr nur $\{111\}$ sondern auch $\{100\}$ - Facetten aufweisen [KoOI00]. Der Vorfaktor $\alpha_{\text {LSW }}$ der Proben ohne Exzeß- O ist damit höher als der der Proben mit Exzeß- O. Dies erklärt den höheren Teilchenradius der Proben ohne Exzeß- O nach gleicher Oxidationsdauer. Für eine genaue Überprüfung dieser Vermutung wäre allerdings eine Untersuchung des Reifungsverhaltens der Proben ohne Exzeß- O für mehrere Glühdauern notwendig.

\subsection{Beschaffenheit der Metall/Oxid (M/O)- Grenzfläche}

Im Verlauf der Experimente zeigte sich, daß es mittels TAP nicht möglich ist, die Grenzfläche der Oxidausscheidungen gezielt zu untersuchen, da diese während der Feldverdampfung einem lokalen Vergrößerungseffekt unterliegen. Im Rahmen dieser Arbeit wurden daher SANS - Untersuchungen an innenoxidierten Ag-1At.\%Mg- und Ag-1At.\%Mn- Proben durchgeführt. Im Wesentlichen wurde dabei das Segregationsverhalten von $\mathrm{H}$, als Sonde zur Charakterisierung der M/O- Grenzfläche, studiert.

\subsubsection{Verifizierung des Kugel- Schale- Modells}

Da die Wasserstoff- Löslichkeit von reinem Silber vernachlässigbar klein ist $\left(\mathrm{c}_{\mathrm{H}}(\mathrm{Ag}) \sim\right.$ $10^{-14} \mathrm{H} / \mathrm{Ag}$ bei $296 \mathrm{~K}$ [FROMM76]), läßt sich eine merkliche H- Löslichkeit des Systems $\mathrm{Ag} / \mathrm{MgO}$ nur mit einer Segregation von Wasserstoff an den $\mathrm{Ag} / \mathrm{MgO}$ - Grenzflächen erklären. Um dies zu überprüfen, kann mit Hilfe der aus dem Kugel- Schale Modell bestimmten $\mathrm{H}$ - Belegungsdichte $\Theta_{\mathrm{H}}$ die aus der Anzahl aller Wasserstoffhaftstellen der

M/O- Grenzfläche resultierende H- Konzentration $\mathrm{c}_{\mathrm{H}}^{\text {Grenzfl. }}$ abgeschätzt werden. Diese bestimmt sich nach

$$
c_{\mathrm{H}}^{\text {Grenzfl. }}=\frac{\Theta_{\mathrm{H}} \cdot \mathrm{S}_{\mathrm{MgO}}}{\mathrm{N}_{\mathrm{Ag}}}
$$


mit $\mathrm{N}_{\mathrm{Ag}}=5,86 \cdot 10^{28} \mathrm{Ag} / \mathrm{m}^{3}, \Theta_{\mathrm{H}}$ (aus Tabelle 3.7 in Kap. 3.2.2) und $\mathrm{S}_{\mathrm{MgO}}$ als totale M/OGrenzfläche der Proben (siehe Tabelle 3.5). Geht man davon aus, daß sich der gesamte Wasserstoff der Probe an den M/O- Grenzflächen befindet und die Matrix- Löslichkeit vernachlässigbar ist, muß der aus (4.2) berechnete Wert gleich der H- Gesamtkonzentration der Probe sein. Letztere wurde aus der inkohärenten Streuung Hbeladener Proben bei großen Q- Werten nach Gl. (2.11) bestimmt und in Tabelle 3.6 vorgestellt. In Abb. 4.5 sind die Werte sowohl für die nach (4.2) berechneten als auch für die aus Tabelle 3.6 bestimmten H- Konzentrationen gegen den mittleren Teilchenradius aufgetragen. Die Werte unterscheiden sich innerhalb der Fehler kaum. Somit befindet sich der gesamte Wasserstoff der Proben an deren inneren $\mathrm{Ag} / \mathrm{MgO}-$ Grenzflächen. Das Kugel- Schale Modell liefert damit eine sehr gute Beschreibung der Streukurven H- (und D-) beladener $\mathrm{Ag} / \mathrm{MgO}$ - Proben. Nach bestem Wissen des Autors stellen die im Rahmen dieser Arbeit durchgeführten Untersuchungen die ersten SANSMessungen dar, die direkte und quantitative Informationen über die Segregation von $\mathrm{H}$ und D an inneren Grenzflächen liefern.

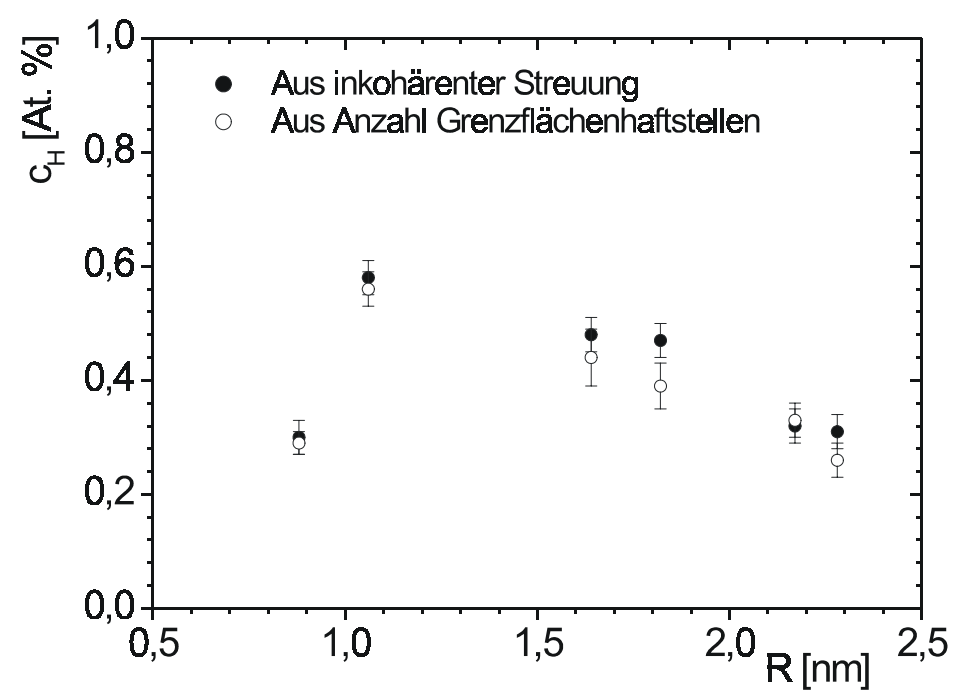

Abb. 4.5: $\quad$ Vergleich der aus der inkohärenten Streuung bestimmten H-Konzentration mit der Anzahl der H-Haftstellen an der Ag/MgO-Teilchengrenzfläche. 


\subsubsection{Segregation und Bindung von $\mathrm{O}$ und $\mathrm{H}$ an der Grenzfläche}

Wie schon in Kap. 3.2.2 beschrieben, ist das Streuverhalten einer H- beladenen Probe nach einer Glühung H- beladener Proben im Hochvakuum $\left(\sim 10^{-2} \mathrm{~Pa}\right)$ unverändert. Dies weist darauf hin, daß H irreversibel in der Probe, bzw. als Ergebnis aus Kap. 4.2.1 an den Teilchengrenzflächen gebunden sein muß.

Der Vergleich der experimentell ermittelten H- Belegungsdichten mit den nach dem strukturellen Modell von Huang, Mader und Kirchheim berechneten H- Belegungsdichten (siehe Kap. 1.2.1) zeigt insbesondere für das System $\mathrm{Ag} / \mathrm{MgO}$ eine sehr gute Übereinstimmung (vgl. Tabelle 4.2). Für das System $\mathrm{Ag} / \mathrm{Mn}_{3} \mathrm{O}_{4}$ liegt die experimentell gefundene $\mathrm{H}$ - Belegungsdichte $\Theta_{\mathrm{H}}{ }^{\text {Exp }}$ etwas höher, als der aus der theoretischen Belegungsdichte des Exzeß- O abgeschätzte Wert $\Theta_{\mathrm{H}}{ }^{\text {Theor. }}$. Im Unterschied zur kohärenten $\mathrm{Ag} / \mathrm{MgO}$ - Grenzfläche [MADER89] bilden $\mathrm{Mn}_{3} \mathrm{O}_{4}-$ Ausscheidungen jedoch semikohärente Grenzflächen zum Ag aus [KOOI00]. Die Anpassungsversetzungen der semikohärenten $\mathrm{Ag} / \mathrm{Mn}_{3} \mathrm{O}_{4}$ - Grenzfläche stellen daher zusätzliche Haftstellen für den Wasserstoff dar, was die Diskrepanz zwischen $\Theta_{\mathrm{H}}^{\text {Exp. }}$ und $\Theta_{\mathrm{H}}{ }^{\text {Theor. }}$ für das System $\mathrm{Ag} / \mathrm{Mn}_{3} \mathrm{O}_{4}$ erklärt.

Tabelle 4.2: $\quad$ Mit SANS bestimmte H-Belegungsdichten im Vergleich zu theoretisch berechneten Werten. Der Wert $\Theta_{H}^{\text {Theor. }}(\mathrm{Ag} / \mathrm{MgO})=2 \Theta_{E x . O}^{\text {Theor. }}(\mathrm{Ag} / \mathrm{MgO})$ basiert auf Annahme eines Flächenverhältnisses von $A^{\{111\}} / A^{\{100\}}=1$ der MgO-Teilchen. $\Theta_{H}^{\text {Exp. }}$ $(\mathrm{Ag} / \mathrm{MgO})$ ist der Mittelwert für $1,6 \mathrm{~nm} \leq R \leq 2,4 \mathrm{~nm}$.

\begin{tabular}{cccc}
\hline System & $\Theta_{H}^{\text {Exp. }}\left[10^{14} \mathrm{~cm}^{-2}\right]$ & $\Theta_{H}^{\text {Theor. }}\left[10^{14} \mathrm{~cm}^{-2}\right]$ & $\Theta_{E x . O}^{\text {Theor. }}\left[10^{14} \mathrm{~cm}^{-2}\right]$ \\
\hline $\mathrm{Ag} / \mathrm{MgO}$ & $7,1 \pm 0,4$ & 6,5 & 3,25 \\
$\mathrm{Ag} / \mathrm{Mn}_{3} \mathrm{O}_{4}$ & $15,0 \pm 2,0$ & 10,8 & 5,4 \\
\hline
\end{tabular}

Die geringere $\mathrm{H}$ - Belegungsdichte des $\mathrm{MgO}$ im Vergleich zum $\mathrm{Mn}_{3} \mathrm{O}_{4}$ läßt sich dadurch erklären, daß MgO- Ausscheidungen nicht nur $\{111\}$ - Grenzflächen sondern auch $\{100\}$ - Grenzflächen aufweisen, an denen kein Exzeß- O und folglich auch kein 
Wasserstoff segregiert (siehe Kap. 1.2.1). Es segregiert in beiden Systemen etwa doppelt so viel Wasserstoff wie Exzeß- $\mathrm{O}$ an der M/O- Teilchengrenzfläche, also:

$$
\Theta_{\mathrm{H}}^{\text {Exp. }} \approx 2 \cdot \Theta_{\text {Ex.O }}^{\text {Theor. }}
$$

Für beide untersuchten Systeme beträgt die H- Belegung der $\{111\}$ - Teilchengrenzflächen damit eine Monolage Wasserstoff. Die Belegungsdichte des Exzeß-O an der $\{111\}$ - orientierten $\mathrm{M} / \mathrm{O}$ - Grenzfläche entspricht damit in Übereinstimmung mit dem strukturellen Modell von Huang, Mader und Kirchheim (siehe Kap. 1.2.1) einer halben Monolage.

$\mathrm{Ag} / \mathrm{MgO}$ - Proben die vor der $\mathrm{H}$ - Beladung einer UHV- Glühung unterzogen wurden, nahmen zudem sehr viel weniger Wasserstoff auf, als Vergleichsproben die keiner vorherigen Glühung im UHV unterzogen wurden. Mit Hilfe der H/D- Kontrastvariation konnte eine Abnahme der $\mathrm{H}$ - Belegungsdichte um $\Delta \Theta_{\mathrm{H}}=4,5 \cdot 10^{14} \mathrm{~cm}^{-2}$ bestimmt werden (siehe Kap. 3.2.2). Die aus der Abnahme des Teilchenradius bestimmte Menge an desorbierten Exzeß- O betrug $\Delta \Theta_{\text {Ex.O }}=2,2 \cdot 10^{14} \mathrm{~cm}^{-2}$ (siehe Kap. 3.2.3). Die Menge der vernichteten $\mathrm{H}$ - Haftstellen entsprach also der doppelten Menge des infolge der UHV- Glühung desorbierten Exzeß-O:

$$
\Delta \Theta_{\mathrm{H}} \approx 2 \cdot \Delta \Theta_{\mathrm{Ex} . \mathrm{O}}
$$

Dieses Ergebnis zeigt den direkten Zusammenhang von Exzeß- O und segregierten Wasserstoff. Sowohl der Vergleich mit der theoretisch berechneten O- Belegungsdichte, als auch die direkte Korrelation von desorbierten Exzeß- O und vernichteten H- Haftstellen infolge der UHV- Glühung, weisen auf eine Bindung des segregierten Wasserstoffs durch den Exzeß- O hin. Dabei werden offensichtlich zwei segregierte H- Atome durch ein Exzeß- O an der M/O- Grenzfläche gebunden. Eine Reaktion an der Grenzfläche könnte somit nach folgender Gleichung ablaufen:

$$
\mathrm{Ag}_{2} \mathrm{O}+\mathrm{H}_{2} \rightarrow \mathrm{H}_{2} \mathrm{O}+2 \mathrm{Ag} \text {. }
$$


Aus den SANS- Messungen allein läßt sich nicht schließen, ob tatsächlich eine Wasserbildung an der M/O- Grenzfläche erfolgt ist. Eine Reaktion unter Bildung von Magnesiumhydroxid (bzw. Manganhydroxid) an der Grenzfläche wäre ebenfalls denkbar

$\mathrm{Ag} / \mathrm{MgO}: \quad \mathrm{MgO}+\mathrm{Ag}_{2} \mathrm{O}+\mathrm{H}_{2} \rightarrow \mathrm{Mg}(\mathrm{OH})_{2}+2 \mathrm{Ag}$

$\mathrm{Ag} / \mathrm{Mn}_{3} \mathrm{O}_{4}: \quad \mathrm{Mn}_{3} \mathrm{O}_{4}+4 \mathrm{Ag}_{2} \mathrm{O}+4 \mathrm{H}_{2} \rightarrow 2 \mathrm{Mn}(\mathrm{OH})_{3}+\mathrm{Mn}(\mathrm{OH})_{2}+2 \mathrm{Ag}$.

Der zuvor als $\mathrm{Ag}_{2} \mathrm{O}$ gebundene Exzeß- O tauscht somit bei Beladung das Silber durch den Wasserstoff als stärkeren Bindungspartner aus, wobei zwei Wasserstoff- Atome pro ein Exzeß- $\mathrm{O}$ an der $\{111\}$-orientierten M/O- Grenzfläche gebunden werden.

Die Menge des infolge der UHV- Glühung desorbierten Exzeß-O $\Delta \Theta_{\text {Ex.O }}$ ist zudem kleiner als die theoretische Belegungsdichte des Exzeß- O $\left(\Delta \Theta_{\text {Ex.O }}<\Theta_{\text {Ex.O }}\right)$. Durch die UHV - Glühung wurde der Exzeß- O also nicht vollständig aus der Probe entfernt. Dies erklärt, weshalb selbst UHV- geglühte Proben eine geringe, aber dennoch meßbare Anzahl irreversibler Haftzentren für den Wasserstoff aufweisen. Nach Gegner ist die Bindungsenergie der Exzeß-O- Atome in der Mitte der $\{111\}$ - Facetten höher, als an deren Kanten [GEGNER95]. Es ist daher anzunehmen, daß bei der durchgeführten UHVGlühung diejenigen Exzeß-O Atome die sich in der Mitte der $\{111\}$ - Facetten befanden nicht desorbiert sind.

\subsection{3 Änderung der Matrixdichte infolge der Segregation}

Aufgrund des höheren Betrages der kohärenten Streulänge von D im Vergleich zu H ist bei Beladung der Grenzfläche die größere Intensitätsänderung für Deuterium zu erwarten und nicht für Wasserstoff. Die Experimente aus Kap. 3.2.2 zeigten jedoch für $\mathrm{Ag} / \mathrm{MgO}$ eine Änderung in $\mathrm{d} \Sigma / \mathrm{d} \Omega$ von $\sim 70 \%$ bei $\mathrm{H}$ - Beladung und nur von $\sim 15 \%$ bei D- Beladung. Dies kann durch eine Änderung der Ag- Matrixdichte infolge der H(D)Segregation an der Grenzfläche interpretiert werden (siehe Kap. 2.2.4). Die AgVerdrängung infolge der H- Segregation konnte mit Hilfe des Kugel- Schale Modells 
(Kap. 2.2.4) ermittelt werden. Die berechneten Werte sind noch einmal in Tabelle 4.3 aufgelistet.

Tabelle 4.3: Ag-Dichteänderung absolut und pro H, sowie notwendige Volumenzunahme $\Delta V_{\text {Exp. }}$ pro Mol segregiertem $\mathrm{H}$. Der Wert für das System $\mathrm{Ag} / \mathrm{MgO}$ gilt für einen Teilchenradius $R>1,1 \mathrm{~nm}$.

\begin{tabular}{cccc}
\hline System & $\Delta \Theta_{A g}\left[\mathrm{~cm}^{-2}\right]$ & $\Delta \Theta_{A g} / \Theta_{H}$ & $\Delta V_{\text {Exp. }}\left[\mathrm{cm}^{3} / \mathrm{mol} \mathrm{H}\right]$ \\
\hline $\mathrm{Ag} / \mathrm{MgO}$ & $6,5 \cdot 10^{14}$ & 0,9 & 9,3 \\
$\mathrm{Ag} / \mathrm{Mn}_{3} \mathrm{O}_{4}$ & $2,7 \cdot 10^{15}$ & 1,8 & 18,5 \\
\hline
\end{tabular}

Für Teilchenradien $\mathrm{R}>1,1 \mathrm{~nm}$ entspricht im System Ag/MgO die Änderung der AgDichte etwa der Menge der segregierten H- Atome. Ein einzelnes segregiertes H- Atom verursacht damit eine Volumenzunahme von fast $100 \%$ des Ag- Atomvolumens. Diese sehr hohe Ag- Verdrängung ist bemerkenswert. Für das System $\mathrm{Ag} / \mathrm{Mn}_{3} \mathrm{O}_{4}$ ist die $\mathrm{Ag}$ Verdrängung sogar doppelt so groß wie die H- Belegungsdichte. Der Wert für $\mathrm{Ag} / \mathrm{Mn}_{3} \mathrm{O}_{4}$ ist allerdings mit einem großen statistischen Fehler behaftet (siehe Kap. 3.4).

Da die hohen Werte von $\Delta \Theta_{\mathrm{Ag}}$ eine starke Volumenzunahme durch H- Segregation implizieren (siehe Tabelle 4.3), werden im Folgenden auf Basis der in Kap. 4.2.2 postulierten Reaktionsgleichungen die theoretischen Volumenzunahmen für das System $\mathrm{Ag} / \mathrm{MgO}$ abgeschätzt. Unter der Annahme, daß die Volumenzunahme aus der Bildung von Wasser bzw. Magnesiumhydroxid an der M/O- Grenzfläche resultiert, berechnen sich folgende Volumenzunahmen der Ag- Matrix pro segregiertem H- Atom:

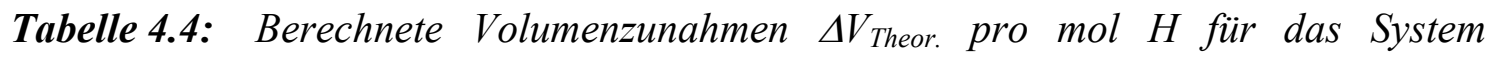
$\mathrm{Ag} / \mathrm{MgO}$. Molvolumina der Reaktanden [cm $/ \mathrm{cmol}^{3}$ : $\mathrm{MgO}$ 11,04; $\mathrm{H}_{2} \mathrm{O} \mathrm{18;} \mathrm{Mg}(\mathrm{OH})_{2} 24,7$ [LANGE99].

\begin{tabular}{lc}
\hline Bildungsprodukt & $\Delta V_{\text {Theor. }}\left[\mathrm{cm}^{3} / \mathrm{mol} \mathrm{H}\right]$ \\
\hline $\mathrm{H}_{2} \mathrm{O}$ & 3,1 \\
$\mathrm{Mg}(\mathrm{OH})_{2}$ & 1,3 \\
\hline
\end{tabular}


Eine Wasserbildung verursacht für beide Systeme eine recht hohe Volumenänderung von $3,1 \mathrm{~cm}^{3} / \mathrm{mol} \mathrm{H}$. Allerdings ist im Fall einer Wasserbildung die höhere Ag- Verdrängung des $\mathrm{Mn}_{3} \mathrm{O}_{4}$ nicht $\mathrm{zu}$ verstehen, da die Volumenzunahme für $\mathrm{Ag} / \mathrm{MgO}$ und $\mathrm{Ag} / \mathrm{Mn}_{3} \mathrm{O}_{4}$ gleich groß ausfällt (vgl. Tabelle 4.3). Im Fall einer Hydroxidbildung fällt der Unterschied zwischen gemessener und $\mathrm{zu}$ erwartender Volumenänderung noch wesentlich größer aus. Somit können weder eine Wasserbildung nach Gl. (4.3) noch eine Hydroxidbildung nach Gl. (4.4) bzw. (4.5) die beobachteten Absolutwerte der AgVerdrängung von $\Delta \Theta_{\mathrm{Ag}} / \Theta_{\mathrm{H}}(\mathrm{MgO}) \approx 1$ und $\Delta \Theta_{\mathrm{Ag}} / \Theta_{\mathrm{H}}\left(\mathrm{Mn}_{3} \mathrm{O}_{4}\right) \approx 2$ erklären. Möglicherweise spielen hier elastische Verzerrungen ebenfalls eine Rolle. Um einen tieferen Einblick in die Volumenänderungen, die mit der H- Segregation verbunden sind, zu bekommen, wären weitere Experimente, wie z. B. Kleinwinkelröntgenstreuungs (SAXS)- Untersuchungen mit Synchrotronstrahlen, erforderlich.

Des weiteren konnte der Effekt einer Änderung der Ag- Matrixdichte nicht nur bei HSegregation, sondern auch bei einer Segregation (bzw. Desorbtion) des Exzeß- O beobachtet werden. Die UHV- Entgasungsexperimente zeigten eine deutliche Abnahme des Exzeß-O-Schalenkontrastes mit zunehmenden Teilchenradius (Tabelle 4.5).

Tabelle 4.5: Abnahme des Exzeß-O Schalenkontrastes $(\Delta \rho \cdot \delta R)_{E x-O}$ mit zunehmenden Teilchenradius $R$.

\begin{tabular}{cc}
\hline$R[\mathrm{~nm}]$ & $(\Delta \rho \cdot \delta R)_{E x-O}\left[\mathrm{~cm}^{-1}\right]$ \\
\hline 1,1 & 150 \\
1,3 & 66 \\
2,4 & 15 \\
\hline
\end{tabular}

Bei einem Teilchenradius von $\mathrm{R}=2,4 \mathrm{~nm}$ verschwindet der Schalenkontrast fast gänzlich. Diese Abnahme kann durch eine Relaxation der Matrix- Atome bei Desorbtion des Exzeß-O erklärt werden. Der Schalenkontrast muß damit nicht nach Gl. (4.6), sondern nach Gl. (4.7) definiert werden:

$$
\begin{array}{ll}
\text { Ohne Relaxaktionsbewegung: } & \left(\Delta \rho_{\mathrm{S}} \cdot \delta \mathrm{R}\right)_{\text {Ex.O }}=\Delta \Theta_{\text {Ex.O }} \cdot \mathrm{b}_{\mathrm{O}} \\
\text { Mit Relaxaktionsbewegung: } & \left(\Delta \rho_{\mathrm{S}} \cdot \delta \mathrm{R}\right)_{\text {Ex.O }}=\Delta \Theta_{\text {Ex.O }} \cdot \mathrm{b}_{\mathrm{O}}-\Delta \Theta_{\mathrm{Ag}} \cdot \mathrm{b}_{\mathrm{Ag}}
\end{array}
$$


Die kohärenten Streulängen von $\mathrm{Ag}$ und $\mathrm{O}$ sind fast identisch $\left(\mathrm{b}_{\mathrm{Ag}}=5,92 \mathrm{fm}\right.$ und $\mathrm{b}_{\mathrm{O}}=$ 5,8 fm). Bei einer gewissen Relaxation von $\Delta \Theta_{\mathrm{Ag}}<\Delta \Theta_{\text {Ex.O }}$ wird der Schalenkontrast $(\Delta \rho \delta \text { R })_{\text {Ex.O }}$ nach Gl. (4.7) damit verschwindend klein. Eine Berechnung der Desorbtionsdichte $\Delta \Theta_{\text {Ex.O }}$ nach Gl. (4.6) liefert damit zu kleine Werte. Die Desorbtionsdichte $\Delta \Theta_{\text {Ex.O }}$ (und damit auch die Exzeß-O- Belegungsdichte) ist allerdings konstant, was auch eine Berechnung von $\Delta \Theta_{\text {Ex.O }}$ aus der Radienabnahme infolge der UHVGlühung zeigt. Dies bedeutet, daß bei Desorbtion eines Exzeß-O eine nahezu vollständige Relaxation der Matrixatome in die entstandene Leerstelle stattfindet. Dieses Ergebnis steht nicht im Einklang mit dem Modell von Huang, Mader und Kirchheim , nach dem die Matrix- Atome keine vollständige Relaxationsbewegung durchführen [HUANG91], da sich bei Desorbtion des Exzeß-O eine strukturelle Leerstelle bildet.

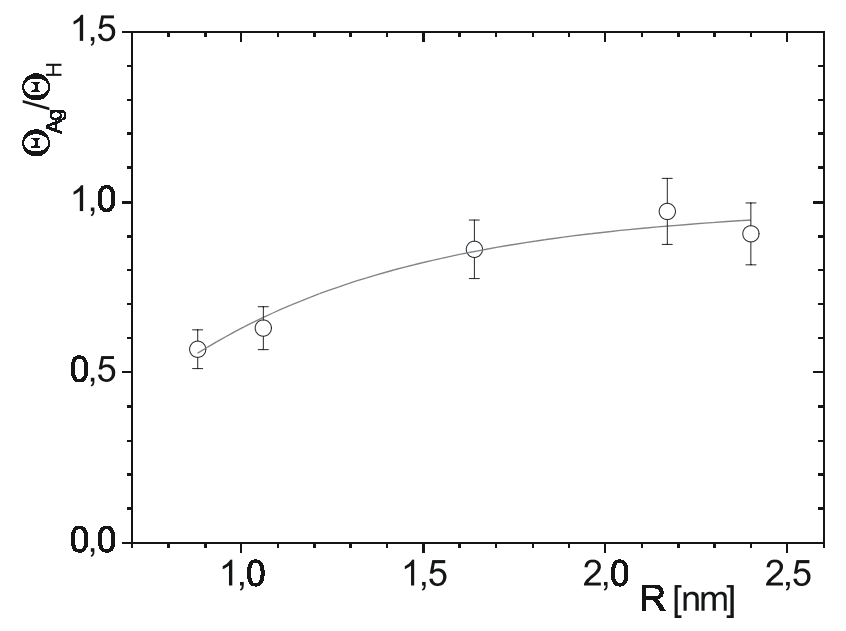

Abb. 4.6: Änderung der Matrixdichte infolge der H-Segregation in Abhängigkeit vom $\mathrm{MgO}$ - Teilchenradius.

Die Zunahme des Exzeß- O- Schalenkontrastes bei kleineren Teilchen (siehe Tabelle 4.5) bedeutet dann eine geringere Relaxation der Matrixatome. Dies ist konsistent mit den Ergebnissen aus Kap. 3.2.2 bzw. der Abb. 4.6. Dort wurde für die Änderung der Matrixdichte durch H- Segregation eine Silberverdrängung von $\Delta \Theta_{\mathrm{Ag}} / \Theta_{\mathrm{H}} \approx 0,5$ für kleine Teilchen mit $\mathrm{R}=0,88 \mathrm{~nm}$ bestimmt. Größere Teilchen $(\mathrm{R}>1,1 \mathrm{~nm})$ wiesen Verdrängungen um $\Delta \Theta_{\mathrm{Ag}} / \Theta_{\mathrm{H}} \approx 1$ auf. Das die Verdrängung für kleine Teilchen geringer ist, folgt direkt aus den in Kap. 3.2.1 gefundenen höheren Grenzflächenrauhigkeiten 
kleinerer Teilchen. Aufgrund des größeren freien Volumina dieser rauhen Grenzflächen, können die bei H- Segregation auftretenden Spannungen leichter abgebaut werden, als bei größeren Teilchen mit scharfen Grenzflächen. Diese höhere Oberflächenrauhigkeit der kleineren Teilchen wird im folgenden Abschnitt noch ausführlicher diskutiert werden.

\subsubsection{Entwicklung der Grenzflächenbeschaffenheit}

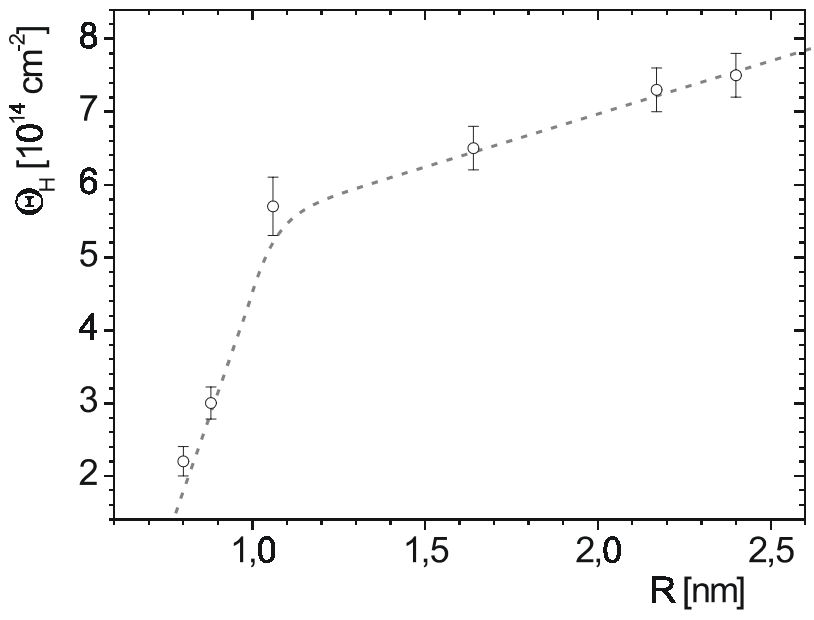

Abb. 4.7: Abhängigkeit der H-Belegungsdichte $\Theta_{H}$ vom Teilchenradius $R$. MgO.

Die aus SANS bestimmten $\mathrm{H}$ - Belegungsdichten $\Theta_{\mathrm{H}}$ des Systems $\mathrm{Ag} / \mathrm{MgO}$ sind in $\mathrm{Abb}$. 4.7 gegen den mittleren Radius der MgO- Ausscheidungen aufgetragen. Für sehr kleine Teilchen von $\mathrm{R}=0,8 \mathrm{~nm}$ beträgt $\Theta_{\mathrm{H}}$ nur etwa ein Drittel des Wertes der größten Teilchen $\left(\Theta_{\mathrm{H}}{ }^{\mathrm{Max}}=7,5 \cdot 10^{14} \mathrm{~cm}^{-2}\right)$. Die H- Belegungsdichte nimmt mit steigender Teilchengröße sehr schnell zu und beträgt bei $\mathrm{R}=1 \mathrm{~nm}$ schon $75 \%$ von $\Theta_{\mathrm{H}}{ }^{\mathrm{Max}}$. Nach dem strukturellen Modell bedeutet dies, daß diese kleinsten Teilchen einen sehr geringen Anteil an $\{111\}$ - Grenzflächen aufweisen, an denen Exzeß-O und somit H segregieren kann. Innerhalb einer geringen Radienzunahme von 0,8 auf $1 \mathrm{~nm}$ nimmt dann offensichtlich der Anteil der $\{111\}$ - Grenzflächen stark zu. Diesen Teilchenradien entsprechen gerade solchen Oxidationszeiten, die im Bereich des Übergangsbereichs von Keimbildung zu Vergröberung liegen (siehe Kap. 4.1.1, Bereich II). Im Rahmen dieser Arbeit wird daher vorgeschlagen, daß in den Frühstadien der IO regelose MgO-Cluster 
gebildet werden, welche dann im Verlauf einer weiteren Glühung zu kohärenten $\mathrm{MgO}$ Ausscheidungen mit facettierten Grenzflächen kristallisieren. Die regelosen Cluster zeichnen sich dabei durch eine sehr geringe H- Belegungsdichte aus, da diese keine definierten $\{111\}$ - Grenzflächen aufweisen. Durch das höhere freie Volumen an den Grenzflächen hat die Segregation von Exzeß- $\mathrm{O}$ und $\mathrm{H}$ an diesen Clustern zudem nur eine geringe Änderung der Ag- Matrixdichte zur Folge (siehe Kap. 4.2.3). Mit zunehmender Glühdauer formieren sich die Cluster dann zu kohärenten MgO- Ausscheidungen, welche facettierte Grenzflächen aufweisen, was die starke Zunahme der H-Belegungsdichte erklärt.

Anschließend folgt für $1 \mathrm{~nm}<\mathrm{R}<2,4 \mathrm{~nm}$ eine stetige aber sehr viel geringere Zunahme von $\Theta_{\mathrm{H}}$ mit steigendem Teilchenradius (vgl. Abb. 4.7). Die Teilchengrößen dieses Bereichs entsprechen Oxidationszeiten $\mathrm{zu}$ denen sich das System schon in der Umlösungsphase befindet (vgl. Abb. 4.2, Bereich III). Berücksichtigt man, daß nur die $\{111\}$ - Grenzflächen Wasserstoff aufnehmen, kann die langsame Zunahme von $\Theta_{\mathrm{H}}$ nur durch eine Zunahme der $\{111\}$ - Facetten auf Kosten der $\{100\}$ - Facetten erklärt werden. Da die Grenzflächenenergie für $\{111\}$ - orientierte Grenzflächen geringer ist als die $\{100\}$ - orientierter $\mathrm{Ag} / \mathrm{MgO}$ - Grenzflächen, bedeutet dies eine gleichzeitige Verringerung der Grenzflächenenergie $\sigma_{\mathrm{Ag} / \mathrm{MgO}}$ mit zunehmendem Teilchenradius. Dieses Ergebnis liefert damit auch eine mögliche Erklärung für die beobachtete Abweichung vom $\mathrm{t}^{1 / 3}$ - Gesetz bei der Vergröberung der MgO- Ausscheidungen (siehe Kap. 4.1.2). 


\section{Zusammenfassung}

In der vorliegenden Arbeit wurden $\mathrm{MgO}$ (bzw. $\mathrm{Mn}_{3} \mathrm{O}_{4}$ )- Ausscheidungen, die sich während der Inneren Oxidation einer Ag-0,4At.\%Mg- (bzw. Ag-0,4At.\%Mn)Legierung bilden, mit Hilfe der Tomographischen Atomsonde (TAP) und der Kleinwinkelneutronenstreuung (SANS) systematisch untersucht.

Ein wesentlicher Teil dieser Arbeit bestand darin, das Segregationsverhalten von Wasserstoff und Deuterium an den mittels Innerer Oxidation hergestellten Ag/MgOund $\mathrm{Ag} / \mathrm{Mn}_{3} \mathrm{O}_{4}$ - Teilchengrenzflächen zu untersuchen. Da die Menge des an den Grenzflächen haftenden Wasserstoffes direkt auf die dortige Menge an Exzeß-O hindeutet, kann der Wasserstoff als Sonde zur Charakterisierung der M/O- Grenzfläche benutzt werden. Leider war es nicht möglich, das Segregationsverhalten von Wasserstoff an der M/O- Grenzfläche mit Hilfe von TAP zu untersuchen. Dies galt sowohl für mittels Innerer Oxidation hergestellte Proben als auch für Metall/Oxid- Schichtpakete die bei H- Beladung versprödeten und während der TAP- Analyse abrissen. Aus diesem Grund wurden SANS- Untersuchungen an den betreffenden Systemen durchgeführt. Das Streuverhalten von H (bzw. D)- beladenen, innerlich oxidierten Proben lies sich dabei hervorragend durch ein sog. Kugel-Schale- Modell beschreiben. Die Ergebnisse zeigten, daß sich der gesamte Wasserstoff der Probe an den M/O- Grenzflächen befindet. Im Rahmen dieser Arbeit wurde damit erstmals SANS erfolgreich benutzt um eine Segregation an inneren Grenzflächen nachzuweisen. Aus UHV- Entgasungsexperimenten konnte nachgewiesen werden, daß je zwei Wasserstoff- Atome durch ein Exzeß- O gebunden werden, wobei sich entweder Wasser oder ein Magnesium (bzw. Mangan)hydroxid bildet. Die H- Segregation verursacht außerdem eine starke Verdrängung der Matrix- Atome an der Grenzfläche. Im System Ag/MgO beträgt die Volumenzunahme an der Grenzfläche infolge der H- Segregation $9,3 \mathrm{~cm}^{3} / \mathrm{mol} \mathrm{H}$, im System $\mathrm{Ag} / \mathrm{Mn}_{3} \mathrm{O}_{4}$ sogar $18,5 \mathrm{~cm}^{3} / \mathrm{mol} \mathrm{H}$. Sowohl für $\mathrm{Ag} / \mathrm{MgO}$ als auch für $\mathrm{Ag} / \mathrm{Mn}_{3} \mathrm{O}_{4}$ wurde die $\mathrm{H}$ Belegungsdichte der $\{111\}$ - Grenzflächen zu einer Monolage bestimmt. Da zwei HAtome durch ein Exzeß-O gebunden werden, beträgt die Belegungsdichte des Exzeß- O an der $\{111\}$ - Grenzfläche folglich eine halbe Monolage. Für beide untersuchten Systeme wurde damit die nach dem strukturellen Modell von Huang, Mader und Kirchheim vorhergesagte Exzeß-O Belegungsdichte beobachtet [HuANG91]. Des 
weiteren konnte gezeigt werden, daß die Desorbtion des Exzeß-O eine nahezu vollständige Relaxation der Matrixatome in die entstandene Leerstelle nach sich zieht. Diese Beobachtung steht nicht im Einklang mit der nach dem strukturellen Modell vorhergesagten Bildung einer Leerstelle bei Desorbtion des Exzeß-O- Atoms [HUANG91].

Für die Untersuchung der Ausscheidungskinetik der Inneren Oxidation einer Ag0,4At.\%Mg- Legierung wurden Auslagerungsexperimente bei einer Oxidationstemperatur von $750{ }^{\circ} \mathrm{C}$ für verschiedene Oxidationszeiten durchgeführt. Die gewonnenen Ergebnisse weisen darauf hin, daß unmittelbar nachdem die Oxidationsfront die Probe durchlaufen hat, alle Mg- Atome durch O- Atome gebunden sind. Nach einer Oxidationsdauer von $t_{I O}=10$ min haben sich durch die Diffusion dieser $\mathrm{MgO}_{\mathrm{x} \geq 1^{-}}$ Moleküle, kleinste $\mathrm{MgO}$ - Ausscheidungen von $\mathrm{R} \approx 0,6 \mathrm{~nm}$ gebildet, welche nach $\mathrm{t}_{\mathrm{IO}}=1$ $\mathrm{h}$ schon einen Radius von 0,9 $\mathrm{nm}$ aufweisen. Bis zu einer Oxidationsdauer von $\mathrm{t}=24 \mathrm{~h}$ wird dann allerdings, sowohl für $\mathrm{MgO}$ - als auch für $\mathrm{Mn}_{3} \mathrm{O}_{4}$ - Teilchen, keine signifikante Größenzunahme der Ausscheidungen beobachtet. Dieses Plateau wird bei der Phasenseparation metallischer Systeme beobachtet und als Übergangsbereich von Keimbildung zu Umlösung diskutiert. Die SANS- Ergebnisse deuten darauf hin, daß sich innerhalb dieses Übergangsbereichs nicht- stöchiometrische, unsymmetrische Oxidcluster zu kohärenten Ausscheidungen kompaktieren. Die Untersuchung der späteren Stadien $\left(t_{I O}\right.$ $\geq 1$ d) zeigt für beide untersuchten Systeme eine deutliche Vergröberung der Oxidteilchen. Es konnte gezeigt werden, daß diese Reifung nicht über eine atomare Diffusion, sondern über eine Diffusion von $\mathrm{MgO}_{\mathrm{X} \geq 1}$ - Molekülen vonstatten geht. Der Diffusionskoeffizient dieser $\mathrm{MgO}_{\mathrm{x} \geq 1^{-}}$Moleküle in $\mathrm{Ag}$ beträgt $\mathrm{D}_{\mathrm{MgO}}\left(750{ }^{\circ} \mathrm{C}\right) \approx 7 \cdot 10^{-16}$ $\mathrm{cm}^{2} / \mathrm{s}$. Für $\mathrm{Ag} / \mathrm{MgO}$ ist der Zeitexponent der Vergröberung mit $\mathrm{n}=0,27$ kleiner als der einer klassischen Ostwaldreifung $(\mathrm{n}=0,33)$. Die SANS- Ergebnisse zeigen, daß bei vergröbernden MgO- Ausscheidungen der Anteil der $\{111\}$ - Grenzflächen auf Kosten der $\{100\}$ - Grenzflächen abnimmt. Da dies eine gleichzeitige Abnahme der Grenzflächenenergie $\sigma_{\mathrm{Ag} / \mathrm{MgO}}$ mit zunehmendem Teilchenradius bedeutet, liefert dieses Ergebnis eine mögliche Erklärung für die beobachtete Abweichung vom $\mathrm{t}^{1 / 3}$ - Gesetz bei der Vergröberung der MgO- Ausscheidungen. 
Abb. 5.1 zeigt schematisch die Veränderung der M/O- Teilchengrenzfläche mit steigendem Teilchenradius $\mathrm{R}$ und zunehmender Oxidationsdauer $\mathrm{t}_{\mathrm{IO}}$. Die Ausscheidungen haben nach der Keimbildung zunächst eine regellose Struktur und kompaktieren sich dann mit zunehmender Glühdauer zu Teilchen mit facettierten Grenzflächen. Bei einer weiteren Glühung vergröbern diese Teilchen dann über eine Diffusion von $\mathrm{MgO}_{\mathrm{x} \geq 1^{-}}$ Einheiten. Mit zunehmender Teilchengröße ändert sich dabei das Verhältnis der $\{111\}$ zu $\{100\}$ - Grenzflächen zu Gunsten der $\{111\}$ - Grenzflächen.

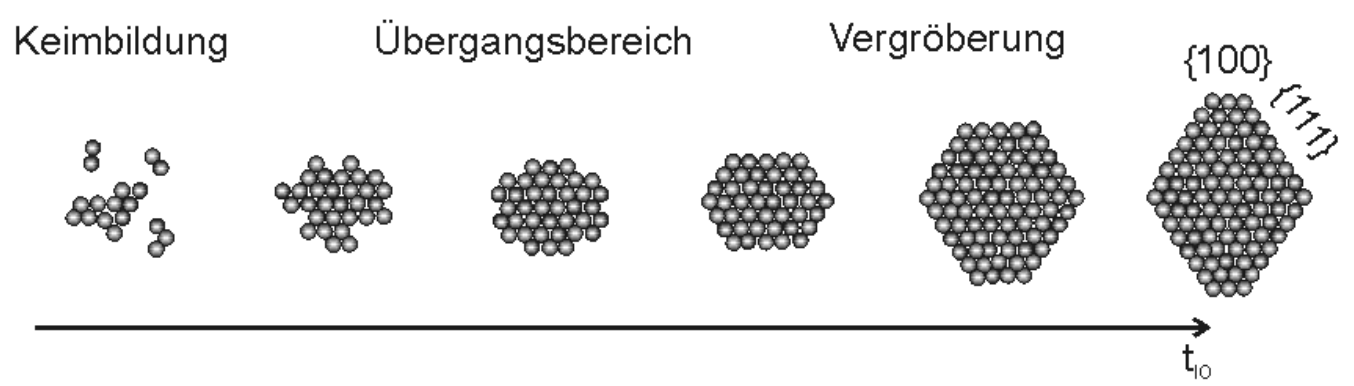

Abb. 5.1: Skizze zur Entwicklung der Teilchenform mit zunehmender Glühdauer. Der Anteil der $\{111\}$ - Grenzflächen nimmt mit längeren Oxidationszeiten zu. 


\section{Literaturverzeichnis}

[AdDa66] Y. Adda, J. Philibert. Diffusion dans les Solides, tome I et II. Presses Universitaires des France, Paris (1996).

[BAckhaus99] M. Backhaus- Ricoult, S. Laurent, J. Devaud; J.Phys. IV 9 (1999) 13.

[BAckhaus00] M. Backhaus- Ricoult, S. Laurent, J. Devaud; Acta Met. Mat. 48 (2000), 4365.

[Beshers58] Beshers, D. N., Acta metal. 6, (1958), 521.

[Bertel03] E. Bertel, C. Deisl, M. Bürgener, G. Meister u. A. Goldmann, Poster im Rahmen der DPG- Frühjahrstagung in Dresden (2003).

[Blavette93] D.Blavette, B.Deconihout, A.Bostel, J.M.Sarrau, M.Bonet, A.Menand, Rev. Sci. Instr. 64 (10) (1993), 2911-2919.

[Blavette99] Blavette, D. Cadel, E., Fraczkiewicz, A. u. Menand, A., Science, 1999, 286, 2317.

[BöHM64] G.Böhm und M.Kahlweit., Vol.12 (1964), S.641-648.

[Chan95] D.K.Chan, D.N.Seidman u. K.L.Merkle. Phy. Rev. Lett. 75/6 (1995), 1118 .

[Charaï88] A. Charaï u. G. Nihoul, Phil. Mag. A, 58, No. 4 (1988), 571-588.

[ChARrin92] L.Charrin, A.Combe, J.Cabané, Oxid. Met. 37 (1992), 65-80.

[Charrin93] L. Charrin, A. Combe, F. Cabané, u. J. Cabane, Oxid. Met. 40 (1993), 483.

[Charrin98] L. Charrin, A. Combe, J. Cabane u. A. Charaï, Rev. Met. (1998), 669.

[ChArrin00] L. Charrin, A. Becquart-Gallissian, A. Combe u. A. Charaï, Scri. Mater. 42 (2000), 701-709.

[COMBE83] A.Combe und J.Cabané, Oxid. Met. 21, Nos.1/2 (1983), 21-37.

[CRANK64] J. Crank, The Mathematics of Diffusion, Oxford University Press (1964), 66-67.

[FÄRBER00] B. Färber, Dissertation, Georg- August- Univ. Göttingen (2000).

[Fromm76] E. Fromm und E. Gebhard, Gase u. Kohlenstoff in Metallen Springer- Verlag, Berlin (1976).

[Fuse02] C. Fuse, Diplomarbeit, Georg- August- Univ. Göttingen (2002).

[GEGNER95] J. Gegner, Dissertation, MPI Stuttgart (1995). 
[GEGNER97] J. Gegner, G.Hörz u. R. Kirchheim, Interf. Science, 5 (1997), 231.

[GuINIER63] A. Guinier, X-Ray Diffraction, Freeman, San Francisco (1963).

[HAASEN87] P.Haasen u. J.Piller. Z.Metallkunde Bd.78 (1987), S.757-766.

[HeInRICh03] A. Heinrich, T. Al-Kassab u. R. Kirchheim, Mat. Sci. Eng. A 353, Iss. 1-2, (2003), 92-98.

[Heuser91] B.J Heuser, J.S King, G.S. Summerfield, F. Boué u. J.E. Epperson, Acta Metal. Mater. 39 (1991), 2815.

[HuAng88] X.Y. Huang, W. Mader, J.A. Eastman u. R. Kirchheim., Scri. Met. 22 (1988), 1109-1114.

[HuAnG91] X.Y. Huang, W. Mader u. R. Kirchheim., Acta Met. 39, No. 5 (1991), 893-907.

[HugGin78] R.O. u. R.A. Huggin, R. Bube u. R. Roberts, Ann. Rev. Mater. Sci. 8 (1978), 327-357.

[JANG92] H. Jang, D.N. Seidman u. K.L. Merkle, Scri. Met. Mat. 26 (1992), 1493-1498.

[KIRCHHEIM81] R. Kirchheim, Acta Met. 29, (1981), 835-845.

[KIRCHHEIM88] R. Kirchheim, Progr. Mat. Sci. 32 (1988), 262.

[Kirchheim03] R. Kirchheim, A. Pundt, T. Al-Kassab, F. Wang, C. Kluthe, Z. Metallkd. 94 (2003), 3.

[Kluthe00] C. Kluthe, Diplomarbeit, Georg- August- Univ. Göttingen (2000).

[Kluthe02] C. Kluthe, T. Al-Kassab and R. Kirchheim, Mat. Sci. Eng. A327 (2002), 70-75.

[Kluthe03A] C. Kluthe, T. Al-Kassab and R. Kirchheim, Mat. Sci. Eng. A 353, Issues 1-2, (2003) 112-118.

[Kluthe03B] C. Kluthe, T. Al-Kassab, J. Barker, W. Pyuckhout-Hintzen u. R. Kirchheim, Eingereicht bei Acta Materialia (2003).

[Kluthe03C] C. Kluthe, M. Ohnuma, T. Al-Kassab und R. Kirchheim, wird veröffentlicht (2003).

[Kool00] B.J. Kooi und J. Th. M De Hosson, Acta Mater. 48 (2000), 36873699.

[Kostorz93] G. Kostorz, Physica Scripta, T49 (1993) 636.

[LANG02] C. Lang, Dissertation, Georg- August- Univ. Göttingen (2002). 
[LANGE99] Lange's Handbook of Chemistry, 15 ${ }^{\text {th }}$ Edition, by J.A. Dean, McGraw Hills Handbooks, (1999), p. 6.93.

[Lifshitz61] I.M. Lifshitz u. V.V. Slyozov; J. Phys. Chem. Solids 19, No. 1/2 (1961), 35 .

[MADER89] W. Mader, Z. Metallkde. 18 (1989), 139-151.

[Mader90] W. Mader u. B. Maier, Coll. de Phys. Coll. C1, Tome 51 (1990), 867-872.

[Makuta79] F. Makuta, Y. Iijima u. K. Hirano, Trans. Jap. Inst. Met. 20, No. 10 (1979), 551-559.

[Maxelon01] M. Maxelon, A. Pundt, W. Pyckhout-Hintzen, J. Barker u. R. Kirchheim, Acta mater. 49 (2001), 2625.

[MeIJERING71] J.L.Meijering. Internal Oxidation in Alloys. Wiley, New York (1971).

[Miller96] M.K.Miller, A.Cereso, M.G.Hetherington u. G.D.W.Smith. Atom Probe Field Ion Microscopy. Clarendon Press, Oxford (1969).

[MÜLLER60] E.W.Müller. Advances in electronics and electron physics. Academic Press, New York 13 (1960), 83.

[Murch91] G.E. Murch; in R.W. Cahn, P. Haasen, E.J. Kramer (Eds.), Mat. Sci. and Techn., Vol. 5, VCH, Weinheim, New York, Basel, Cambridge (1991), 74.

[MütschelL87] T. Mütschell u. R. Kirchheim, Scri. Metal. 21 (1987), 1101-1104.

[NoH96] H. Noh, T. Flanagan, R. Balasubramaniam u. J. Eastman, Scri. Mat. 34, (1996), 863-868.

[Opara97] L. Opara, B. Klein, H. Züchner, J. Al. and Comp. 253/254 (1997), 378-380.

[ORTH96] U. Orth, Diplomarbeit, Georg- August- Univ. Göttingen (1996).

[PIPPEL00] E. Pippel, J. Woltersdorf, J. Gegner, R. Kirchheim, Acta Mat. 48 (2000), 2571-2578.

[PedDER78] D.J. Pedder, Met. Trans. A, 9A (1978), 659-670.

[Glatter83] Porod, G., in Small-Angle X-ray Scattering, ed. O. Glatter and O. Kratky,. Acandemic Press, New York, (1983).

[RAmanara72] T.A. Ramanarayanan u. R.A. Rapp, Metal. Trans. 3 (1972), 32393246. 
[RIKEL99] M.O. Rikel, W. Goldacker. J. Mater. Res. 14, No. 6 (1999), 24362445 .

[RoвACH97] O. Robach, G. Renaud, A. Barbier u. P. Guénard, Surf.Rev.Lett. 5, No. 1 (1997),359-362.

[RÜSING00] J. Rüsing, J.T. Sebastian, O.C. Hellman u. D.N. Seidman, Microscopy u. Microanalysis 6(5), (2000), 445-451.

[SHEwmon69] P.G. Shewmon, Transformation of Metals, New York, McGraw-Hill (1969).

[SCHLEIWIES01] J. Schleiwies, Dissertation, Georg- August- Univ. Göttingen (2001).

[SCHMALZRIED95]H.Schmalzried. Chemical Kinetics of Solids. VCH Verlagsgesellschaft, Weinheim (1995).

[Semega92] B.M. Semega, L. Charrin, A. Combe u. J. Aridl, Phil. Mag. A. 66, No. 6 (1992), 1139-1148.

[SpREnGler65] H. Sprengler, Metallw. und Technik 19, Heft 7 (1965), 725-728.

[Vitos98] L. Vitos, A.V. Ruban, H.L. Skriver, J. Kollár, Surf. Sci. 411 (1998), 186-202.

[Vurpillot00] F. Vurpillot, A. Bostel und D. Blavette, Appl. Phys. Lett. 76, No. 21 (2000), 3127.

[WAGNER59] C. Wagner, Z. Elektrochem. 63/7 (1959), 772-782.

[WAGNER61] C. Wagner, Z. Elektrochem. 65, No. 7/8 (1961), 581.

[WAgneR91] R. Wagner u. R. Kampmann; in R.W. Cahn, P. Haasen, E.J. Kramer (Eds.); Mat. Sci. and Techn. 5, VCH, Weinheim (1991), 213.

[WeinKamer01] R. Weinkamer u. P. Fratzl, Vortrag im Rahmen der DPG- Frühjahrstagung in Hamburg (2001).

[Wolde03] D. Wolde-Giorgis, T. Al-Kassab and R. Kirchheim, Mat. Sci. Eng. A 353, Issues 1-2, (2003), 152-157.

[XIAO89] Xiao, S. Q. and Haasen, P., Scripta Met. 23, (1989), 295 u. 365. 


\section{Appendix}

\subsection{Streuquerschnitt einer segregierten Schale}

Im Folgenden wird eine Herleitung des makroskopischen Streuquerschnitts für den Spezialfall einer segregierten Schale gegeben. Für gewöhnlich wird die gestreute Intensität durch folgenden Ausdruck beschrieben

$$
\frac{\mathrm{d} \Sigma}{\mathrm{d} \Omega}=\mathrm{N}\left|\int \Delta \rho \exp (-\mathrm{iQr}) \mathrm{d}^{3} \mathrm{r}\right|^{2}=16 \pi^{2} \mathrm{~N}|\mathrm{P}(\mathrm{Q})|^{2}
$$
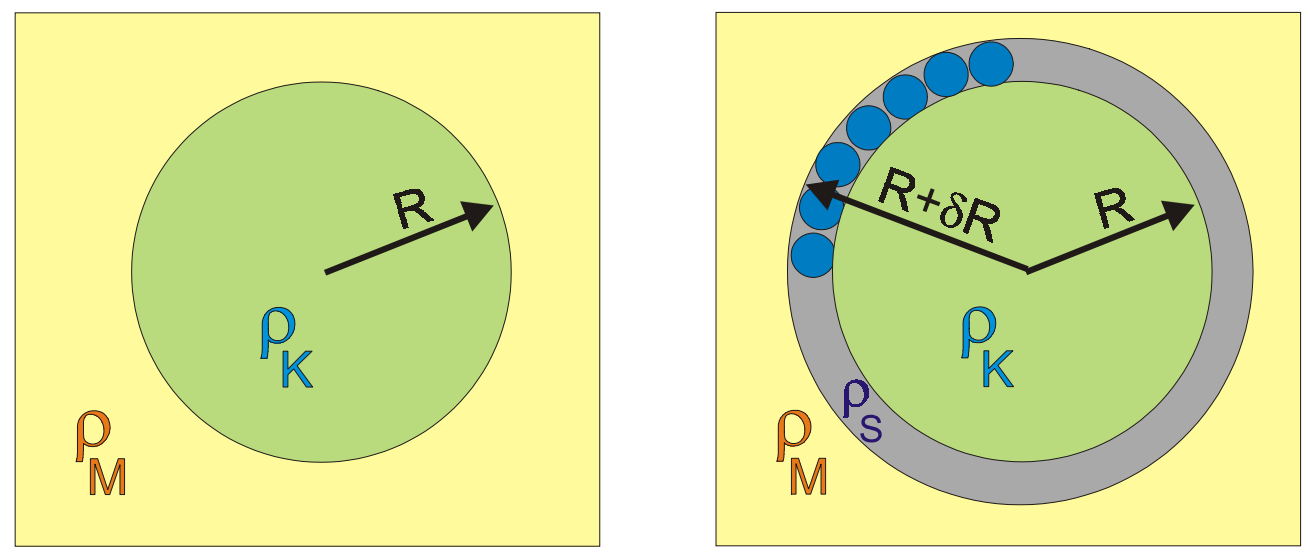

Abb. 6.1: Skizze zum Kugel-Schale Modell. Rechts ohne, links mit segregierter Schale.

$\mathrm{N}$ ist die Anzahl der streuenden Objekte, $\Delta \rho$ die Streulängendichtedifferenz, i.a. Anzahl der Streuer mal der kohärenten Streulänge $b_{n}$ pro Einheitsvolumen, mit Q als Streuvektor. Der makroskopische Streuquerschnitt kann, wie in Gl. (6.1) zu sehen, durch den Formfaktor $\mathrm{P}(\mathrm{Q})$ der streuenden Objekte ausgedrückt werden. Im Folgenden wird der Formfaktor einer Kugel sowie einer Kugel mit einer zusätzlichen Schale präsentiert. Der Kugel- Formfaktor $\left.\mathrm{P}(\mathrm{Q})\right|_{\text {Kugel }}$ ist bekannt und entspricht

$$
\left.\mathrm{P}(\mathrm{Q})\right|_{\text {Kugel }}=\Delta \rho_{\mathrm{KM}} \cdot \mathrm{f}(\mathrm{R}) \quad \mathrm{f}(\mathrm{R})=\frac{\sin (\mathrm{QR})-\mathrm{QR} \cos (\mathrm{QR})}{\mathrm{Q}^{3}}
$$


$\Delta \rho_{\mathrm{KM}}$ ist die Streulängendichtedifferenz von Kugel zu Matrix und R der Kugel- oder Teilchenradius. Mit Hilfe dieses Kugel- Formfaktors läßt sich nach Guinier folgende Näherung für kleine Q- Vektoren herleiten [GUINIER63]:

$$
\left.\frac{\mathrm{d} \Sigma}{\mathrm{d} \Omega}\right|_{\text {Kugel }} \cong \frac{16}{9} \pi^{2} \mathrm{~N} \cdot \mathrm{R}^{6} \Delta \rho_{\mathrm{KM}}^{2} \cdot \exp \left(-\frac{\mathrm{Q}^{2} \mathrm{R}^{2}}{5}\right) .
$$

Für eine Kugel mit einer zusätzlichen äußeren Schale wird der Formfaktor zu

$\left.\mathrm{P}(\mathrm{Q})\right|_{\text {Kugel }+ \text { Schale }}=\Delta \rho_{\mathrm{S}} \cdot \mathrm{f}(\mathrm{R}+\delta \mathrm{R})-\Delta \rho_{\mathrm{S}} \cdot \mathrm{f}(\mathrm{R})+\Delta \rho_{\mathrm{KM}} \cdot \mathrm{f}(\mathrm{R})$,

hierbei ist $\Delta \rho_{\mathrm{S}}$ die Streulängendichtedifferenz einer Schale segregierter Atome zu Vakuum. Eine Entwicklung von $\mathrm{f}(\mathrm{R}+\delta \mathrm{R})$ unter Vernachlässigung quadratischer Anteile führt auf

$\left.\mathrm{P}(\mathrm{Q})\right|_{\mathrm{K}+\mathrm{S}}=\Delta \rho_{\mathrm{S}} \cdot \delta \mathrm{R} \cdot \mathrm{df}(\mathrm{R}) / \mathrm{dR}+\Delta \rho_{\mathrm{KM}} \cdot \mathrm{f}(\mathrm{R})$

Der makroskopische Streuquerschnitt $\mathrm{d} \Sigma / \mathrm{d} \Omega$ wird so zu

$$
\left.\frac{\mathrm{d} \Sigma}{\mathrm{d} \Omega}\right|_{\text {Kugel }+ \text { Schale }}=16 \pi^{2} \mathrm{~N}|\mathrm{P}(\mathrm{Q})|^{2}=16 \pi^{2} \mathrm{~N}\left|\Delta \rho_{\mathrm{S}} \cdot \mathrm{f}^{\prime}(\mathrm{R}) \cdot \delta \mathrm{R}+\Delta \rho_{\mathrm{KM}} \cdot \mathrm{f}(\mathrm{R})\right|^{2}
$$

Unter Vernachlässigung quadratischer Terme in $\delta \mathrm{R}$ erhält man

$$
\left.\frac{\mathrm{d} \Sigma}{\mathrm{d} \Omega}\right|_{\mathrm{K}+\mathrm{S}} \cong 16 \pi^{2} \mathrm{~N}\left[2 \Delta \rho_{\mathrm{S}} \Delta \rho_{\mathrm{KM}} \delta \mathrm{R} \cdot \mathrm{f}^{\prime} \mathrm{f}+\Delta \rho_{\mathrm{KM}}^{2} \cdot \mathrm{f}^{2}\right]
$$

wobei $\frac{d f}{d R}=\frac{Q^{2} R \sin (Q R)}{Q^{3}}$ und $f(R)=\frac{\sin (Q R)-Q R \cos (Q R)}{Q^{3}}$.

Aus der Differenz von Gl. (6.5) und (6.2) erhält man dann den Netto- Streuquerschnitt der segregierten Schale 


$$
\left.\frac{\mathrm{d} \Sigma}{\mathrm{d} \Omega}\right|_{\mathrm{K}+\mathrm{S}}-\left.\frac{\mathrm{d} \Sigma}{\mathrm{d} \Omega}\right|_{\mathrm{K}}=16 \pi^{2} \mathrm{~N} \Delta \rho_{\mathrm{KM}} 2 \delta \mathrm{R} \Delta \rho_{\mathrm{S}} \frac{\left[\mathrm{Q}^{2} \mathrm{R} \sin (\mathrm{QR})\right][\sin (\mathrm{QR})-\mathrm{QR} \cos (\mathrm{QR})]}{\mathrm{Q}^{6}} .
$$

Der erste Term im Bruch nimmt mit $\mathrm{Q}^{-4}$ ab und der zweite oszilliert um Null. Für den Nettostreuquerschnitt der segregierten, äußeren Schale erhält man dann folgende Näherung für den Guinier- Bereich (Entwicklung nach QR bis zu Termen zweiten Grades und Umwandlung in eine Exponentialfunktion):

$$
\left.\frac{\mathrm{d} \Sigma}{\mathrm{d} \Omega}\right|_{\text {Schale }}=\left.\frac{\mathrm{d} \Sigma}{\mathrm{d} \Omega}\right|_{\mathrm{K}+\mathrm{S}}-\left.\frac{\mathrm{d} \Sigma}{\mathrm{d} \Omega}\right|_{\mathrm{K}} \cong \frac{32}{3} \pi^{2} \mathrm{~N} \Delta \rho_{\mathrm{KM}} \mathrm{R}^{5} \delta \mathrm{R} \Delta \rho_{\mathrm{S}} \cdot \exp \left(-\frac{4 \mathrm{Q}^{2} \mathrm{R}^{2}}{15}\right) .
$$

Außerdem kann man den normierten Nettostreuquerschnitt $\mathrm{d} \Sigma /\left.\mathrm{d} \Omega\right|_{\text {Schale }} ^{0}$ bilden, indem man Gl. (6.7) mit dem Streuquerschnitt der reinen Kugel Gl. (6.3) normiert

$$
\left.\frac{\mathrm{d} \Sigma}{\mathrm{d} \Omega}\right|_{\text {Schale }} ^{0}=\frac{6 \Delta \rho_{\mathrm{S}} \delta \mathrm{R}}{\Delta \rho_{\mathrm{KM}} \mathrm{R}} \cdot \exp \left(-\frac{\mathrm{Q}^{2} \mathrm{R}^{2}}{15}\right)
$$

$\mathrm{d} \Sigma /\left.\mathrm{d} \Omega\right|_{\text {Schale }} ^{0}$ ist der normierte Nettostreuquerschnitt einer segregierten, äußeren Schale. Gl. (6.8) stellt eine Näherung für den Guinier- Bereich dar. 


\subsection{Gesputterte Metall/Oxid- Schichten}

\subsubsection{Präparation der Schichtpakete}

Als Substrat für die Beschichtung dienten W-Spitzen, die elektrolytisch aus einem W-Draht gedünnt wurden. Um eine reproduzierbare Spitzenoberfläche zu erhalten, wurden die Spitzen anschließend im FIM bis zu einer Spannung von $9 \mathrm{kV}$ bei $100 \mathrm{~K}$ entwickelt. Die anschließende Beschichtung wurde in der in Abb. 6.2

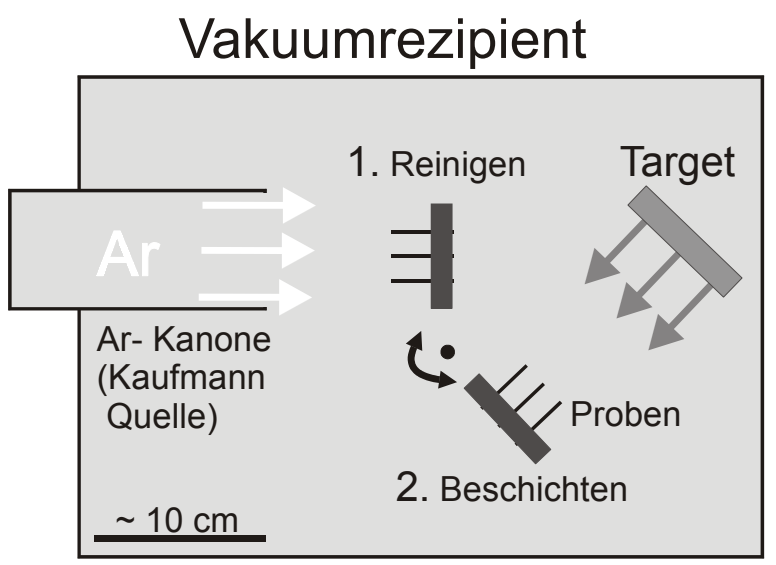
dargestellten Kammer durchgeführt.

Abb. 6.2: Geometrie Beschichtungskammer. Ein Ar-Ionenstrahl dient in dieser sowohl zur Reinigung als auch zum Abtrag (Sputtern) des Targetmaterials [SCHLEIWIES01]. Die experimentellen Parameter zur Reinigung und Beschichtung finden sich in Tabelle 6.1. Sowohl Proben als auch Targets werden auf eine Temperatur von $18^{\circ} \mathrm{C}$ gekühlt. Die Depositionsrate läßt sich mit einem Schwingquarz kontrollieren.

Tabelle 6.1: Beschichtungsparameter. Typische Depositionsraten lagen zwischen 0,5 und 1,5 A/sec. Die Entladespannung $U_{E}$ bestimmt die Divergenz des Ionenstrahls (55V: Strahl parallel, $45 \mathrm{~V}$ : leicht divergent). Der Ar-Sputterdruck betrug 10-2 Pa, der Restgasdruck $<10^{-5} \mathrm{~Pa}$.

\begin{tabular}{l|ccccc}
\hline Vorgang & $I_{\text {Beam }}[\mathrm{V}]$ & $I_{\text {Beam }}[\mathrm{A}]$ & $j_{\text {Beam }}\left[\mathrm{A} / \mathrm{cm}^{2}\right]$ & $U_{E}[\mathrm{~V}]$ & Zeit $t$ \\
\hline Targetreinigung & 600 & 25 & 3,5 & 55 & je $2 \mathrm{~min}$ \\
Substratreinigung & 500 & 10 & 1,4 & 45 & $30 \mathrm{~s}$ \\
Beschichtung & 600 & 20 & $0,8-2,1$ & 55 & $10 \mathrm{~s}-5 \mathrm{~min}$ \\
\hline
\end{tabular}


Da zu erwarten ist, daß das Segregationsverhalten von Gasen stark von der Orientierung der Grenzfläche abhängt (Kap. 1.2), wurde versucht, gezielt Schichten bestimmter Orientierung herzustellen (siehe Abb. 6.3). Die Dicke der Schichten betrug je 50
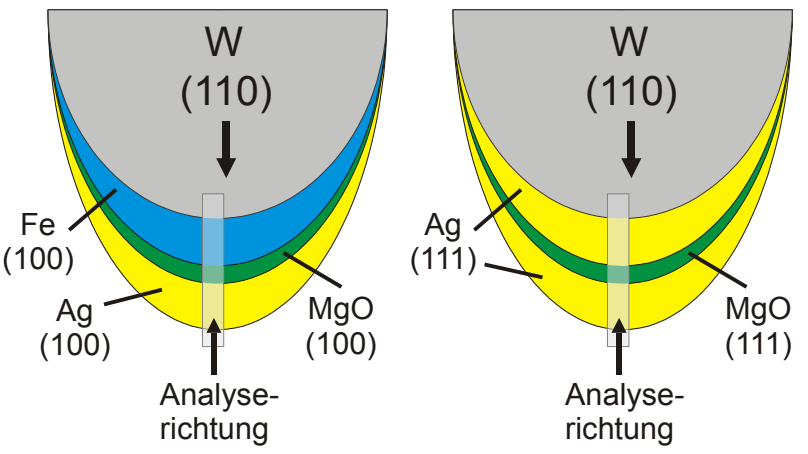

Abb. 6.3: Skizze der Schichtgeometrien. nm für $\mathrm{Ag}$ und Fe sowie etwa 1 $\mathrm{nm}$ für das $\mathrm{MgO}$. Das $\mathrm{MgO}$ wurde direkt von einem $\mathrm{MgO}$ - Target deponiert. Aufgrund der hohen Reaktivität des Magnesiums bilden sich ohne Einstellen eines zusätzlichen OPartialdrucks MgO- Schichten in perfekter 1:1 Stöchiometrie [FusE02]. Wie in Abb. 6.3 angedeutet, sollte sich bei Deposition von Fe auf (110)- orientiertem W eine $\{100\}$ orientierte Fe- Schicht bilden. Nach Fuse wächst bei Laser- Deposition von MgO dieses epitaktisch auf $\{100\}$ - orientiertem Eisen auf [FUSE02]. Aus Röntgenuntersuchungen ist bekannt, daß Ag auf $\{100\}$ - orientiertem MgO epitaktisch aufwächst [ROBACH97]. Auf diese Art kann somit eine $\{100\}$ - orientierte Ag/MgO- Grenzfläche hergestellt werden.

Für die Herstellung $\{111\}$ - orientierter $\mathrm{Ag} / \mathrm{MgO}$ - Grenzflächen wird ausgenutzt, daß $\mathrm{Ag}$ in [111]- Richtung auf (110)- orientiertem Wolfram aufwächst [BERTEL03, SCHLEIWIES01]. Es ist anzunehmen, daß ein dünner MgO- Film von etwa $1 \mathrm{~nm}$ Dicke ebenfalls in [111]- Richtung aufwächst. Eine weitere Ag- Schicht besitzt dann auch eine [111]- Orientierung.

\subsubsection{TAP- Analysen der Schichtpakete}

In Kap. 3.1.2 zeigte sich, daß bei der TAP- Analyse von oxidischen Ausscheidungen eine Identifizierung des segregierten Wasserstoffs nicht möglich ist, da deren Feldverdampfung nicht homogen verläuft. Motiviert durch die Annahme, daß die Feldverdampfung bei einer geschlossenen, aber sehr dünnen Oxidschicht wesentlich homogener abläuft, wurden ebenfalls Oxidschichten untersucht. Ein weiterer Vorteil gegenüber der Analyse oxidischer Ausscheidungen ist die Möglichkeit einer Rekonstruktion des 
Analysevolumens auf Basis des Krümmungsradius und Schaftwinkels der FIM- Spitze. Diese Art der Rekonstruktion ist unempfindlich gegenüber Veränderungen der Feldverdampfungsfeldstärke während der Analyse. Der Algorithmus ist anwendbar solange es sich um einzelne, aufeinanderfolgende Schichten handelt, die während der Analyse nacheinander feldverdampfen. Für eine ausführlichere Beschreibung sei an dieser Stelle auf [LANG02] verwiesen. Abb. 3.5a,b zeigen die rekonstruierten Analysevolumina der so hergestellten $\mathrm{Fe} / \mathrm{MgO} / \mathrm{Ag}$ - sowie $\mathrm{Ag} / \mathrm{MgO} / \mathrm{Ag}$ - Schichtpakete. Beide Messungen zeigen eine, innerhalb des Analysevolumens geschlossene, $\mathrm{MgO}$ - Schicht von etwa 1 nm Dicke. Die im Rahmen dieser Arbeit durchgeführten Messungen oxidischer Schichtpakete stellen die ersten erfolgreichen TAP- Messungen oxidischer Schichtpakete dar.

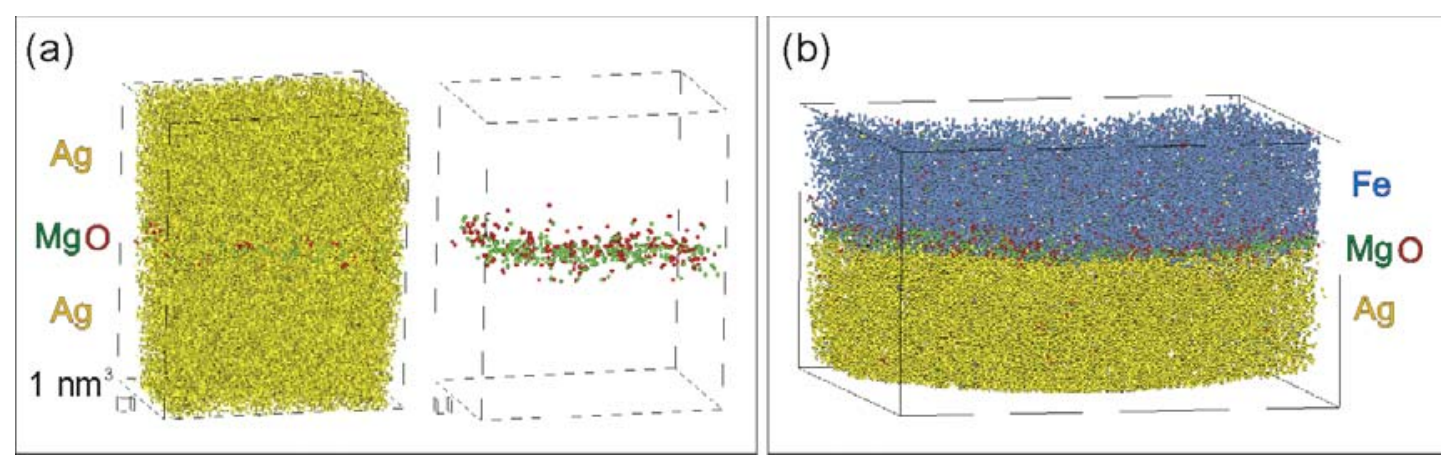

Abb. 6.4: Rekonstruierte Analysevolumina; (a) Ag/MgO/Ag-Schichtpaket, links: alle Atome, rechts: nur $\mathrm{Mg}$ - und O-Atome; (b) Fe/MgO/Ag-Schichtpaket. Boxgröße: $18 \times 18 \times 10,5 \mathrm{~nm}$.

Die Feldverdampfung der Schichtpakete verläuft wesentlich homogener als bei der Analyse von MgO- Ausscheidungen in Ag. Die Massenspektren der TAP- Analysen der Schichtpakete weisen einen deutlich geringeren Anteil an Restgas- Wasserstoff auf, als im Fall der Analyse oxidischer Teilchen (Abb. 6.5). Bei der Analyse oxidischer Ausscheidungen fand sich sogar $\mathrm{H}_{3}{ }^{+}$im Spektrum wieder. Für oxidische Schichten ist eine Identifizierung des segregierten Wasserstoffs deshalb vergleichsweise einfacher als bei der Analyse oxidischer Ausscheidungen. Bedauerlicherweise wird die Stabilität der Spitzen durch die Wasserstoffbeladung derart gering, daß keines der mit Deuterium beladenen $\mathrm{Ag} / \mathrm{MgO} / \mathrm{Ag}$ - Schichtpakete erfolgreich im TAP gemessen werden konnte. Alle D- beladenen Spitzen rissen immer nach einigen 10000 detektierten Ag- Ionen ab. Es muß daher festgestellt werden, daß eine Untersuchung des Segregationsverhaltens von Wasserstoff zur Charakterisierung der M/O- Grenzfläche nicht mit TAP durch- 
geführt werden kann. Dies gilt sowohl für $\mathrm{MgO}$ - Ausscheidungen in $\mathrm{Ag}$ als auch für $\mathrm{MgO}-$ Schichten.

(a)

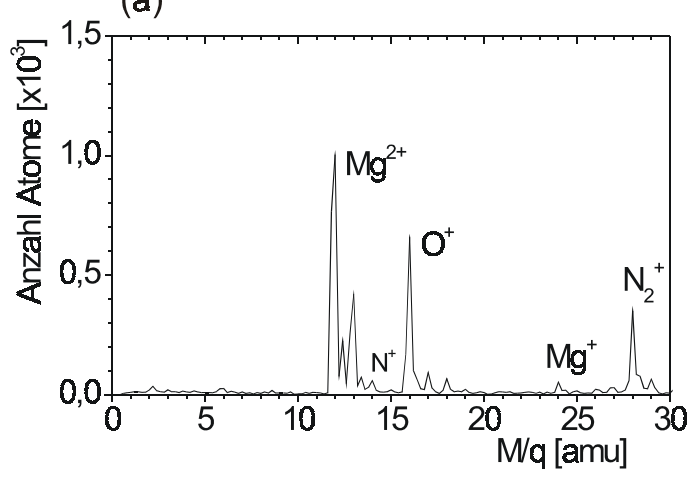

(b)

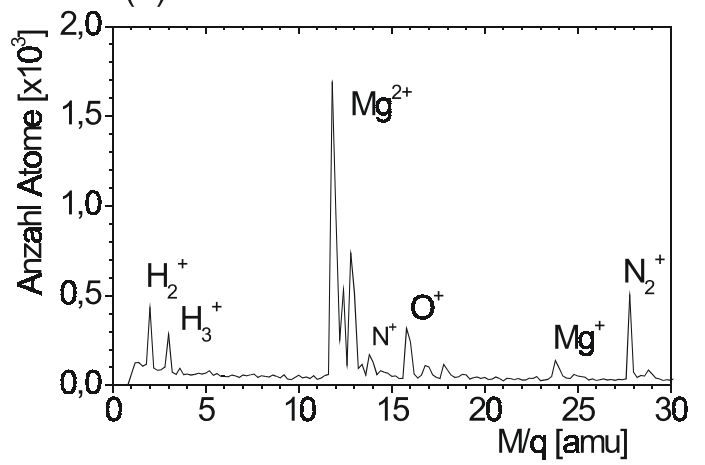

Abb. 6.5: Massenspektren im Bereich kleiner Massen; (a) Ag/MgO/Ag- Schichtpaket (b) innenoxidierte Ag-0,4At.\%Mg-Legierung.

Abb. 6.6a,b zeigen die Konzentrationsanalysen der Schichtpakete aus Abb. 3.5a,b. Im Fall einer geschlossenen reinen $\mathrm{MgO}-$ Schicht sollte die Ag- bzw. Fe- Konzentrationen innerhalb der $\mathrm{MgO}$ - Schicht auf Null abfallen. Allerdings findet sich sowohl für $\mathrm{Ag} / \mathrm{MgO} / \mathrm{Ag}$ als auch für das $\mathrm{Fe} / \mathrm{MgO} / \mathrm{Ag}$ - Schichtpaket selbst innerhalb der MgOSchicht eine gewisse Ag- bzw. Fe- Konzentration. Teilweise läßt sich dieses Ergebnis durch einen sogenannten „Sampling“- Effekt begründen. Da die Schichten sehr dünn und zudem noch leicht gekrümmt sind, die Konzentrationsanalyse jedoch in ebenen Schnitten entlang der Z- Richtung vollzogen wird, ist es nicht möglich die maximale Konzentration der Barriere zu treffen. Die sehr geringe $\mathrm{Mg}$ - und O- Konzentration des $\mathrm{Ag} / \mathrm{MgO} / \mathrm{Ag}-$ Schichtpaketes innerhalb der MgO- Schicht kann durch diesen Effekt allerdings nicht erklären werden. Es ist davon auszugehen, daß es sich in diesem Fall nicht um eine geschlossene Schicht aus $\mathrm{MgO}$ handelt.

Eine Konzentrationsanalyse des $\mathrm{Ag} / \mathrm{MgO} / \mathrm{Ag}$ - Schichtpaketes in Abb. 3.5a senkrecht zu der $\mathrm{MgO}$ - Schicht zeigt eine breitere Verteilung des Sauerstoffs im Vergleich zum Mg (Abb. 6.6a). Das O/Mg- Verhältnis innerhalb der $\mathrm{MgO}-$ Schicht beträgt $\mathrm{O} / \mathrm{Mg}=1,10 \pm$ 0,03. Die Halbwertsbreite der Mg- Verteilung beträgt $d_{M g}=0,7 \pm 0,1 \mathrm{~nm}$, die der OVerteilung $\mathrm{d}_{\mathrm{O}}=1,2 \pm 0,2 \mathrm{~nm}$. Da die O- Konzentration oberhalb der 1:1- Stöchiometrie des $\mathrm{MgO}$ liegt ist davon auszugehen, daß ein Teil des Sauerstoffs auch mit Silber- 
Atomen nahe der $\mathrm{Ag} / \mathrm{MgO}$ - Grenzflächen eine Bindung eingeht oder zumindest dort gelöst ist.

(a)

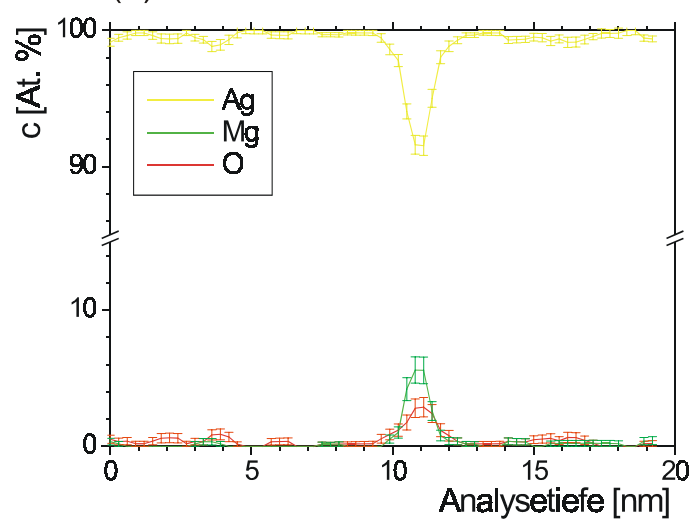

(b)

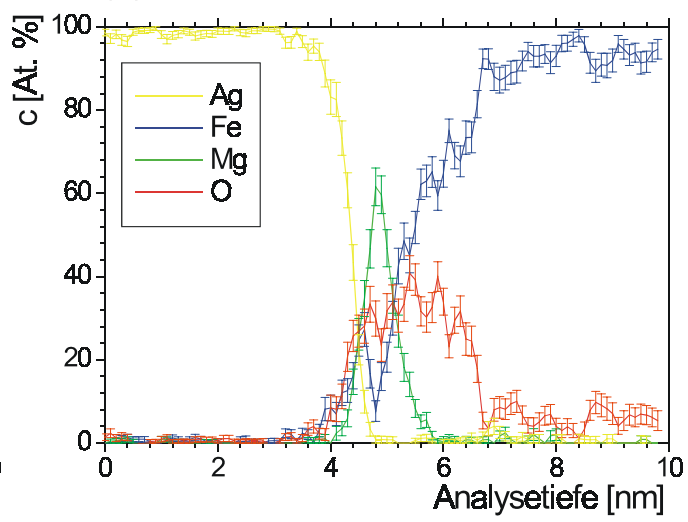

Abb. 6.6: Konzentrationsanalysen senkrecht zur $\mathrm{MgO}$ - Schicht; (a) $\mathrm{Ag} / \mathrm{MgO} / \mathrm{Ag}$ Schichtpaket. Die Halbwertsbreite der Mg-Verteilung beträgt $d_{M g}=0,7 \mathrm{~nm}$. (b) $\mathrm{Fe} / \mathrm{MgO} / \mathrm{Fe}$-Schichtpaket, $d_{\mathrm{Mg}}=0,6 \mathrm{~nm}$.

Für das $\mathrm{Ag} / \mathrm{MgO} / \mathrm{Fe}-$ Schichtpaket reichert sich scheinbar Fe innerhalb der Grenzfläche $\mathrm{Ag} \mathrm{zu} \mathrm{MgO}$ an. Bei dieser scheinbaren Fe- Anreicherung handelt es sich vermutlich um Stickstoff des Restgases, da $\mathrm{N}_{2}{ }^{+}$- Ionen und $\mathrm{Fe}^{2+}$ - Ionen bei 28 amu einen Massenüberlapp haben. Diese Interpretation wird gestützt von der Tatsache, daß auch im Massenspektrum des $\mathrm{Ag} / \mathrm{MgO} / \mathrm{Ag}$ - Schichtpaketes ein Peak bei M/q = 28 amu auftritt, welcher hier eindeutig den $\mathrm{N}_{2}{ }^{+}$- Ionen zugeordnet werden kann (siehe Abb. 6.5). Durch den hohen Unterschied der Feldverdampfungsfeldstärken kondensiert der Stickstoff im Verlauf der TAP- Analyse am Apex der Spitze, feldverdampft dann wieder von dieser und findet sich daher im Massenspektrum der Messung wieder. Der Sauerstoff reicht in erhöhter Konzentration etwa 1,2 $\mathrm{nm}$ in die Fe- Schicht hinein. Dies bedeutet, daß im as sputtered-Zustand etwa 6 Monolagen des Eisens ebenfalls oxidiert wurden, obwohl das Eisen vor dem $\mathrm{MgO}$ deponiert wurde. Die Halbwertsbreite der Mg- Verteilung beträgt $\mathrm{d}_{\mathrm{Mg}}=0,6 \pm 0,1 \mathrm{~nm}$, die der O- Verteilung $\mathrm{d}_{\mathrm{O}}=4,1 \pm 0,2 \mathrm{~nm}$. Auch auf der Grenzfläche des $\mathrm{MgO}$ zum Ag fällt die Mg- Konzentration schneller ab, als die des Sauerstoffs (Abb. 6.6b). 


\section{Danksagung}

An erster Stelle gilt mein Dank Herrn Prof. Dr. Reiner Kirchheim für die Ermöglichung einer Dissertation am Institut für Materialphysik und für den Freiraum bei der Ausgestaltung dieser Arbeit. Sein großes Interesse am Fortgang dieser Arbeit war für mich sehr motivierend.

Herrn Dr. Talaát Al-Kassab danke ich für die Betreuung, sowie die vielen Anregungen und Diskussionen zum Thema dieser Arbeit. Er stand mir während der gesamten Zeit stets hilfreich zur Seite.

Herrn Dr. Wim Pyckhout-Hintzen vom FZ Jülich, Dr. John Barker, Dr. Juscelino Leão und Dr. Terry Udovic vom NIST in Gaithersburgh M.D. (USA) möchte ich für die unkomplizierte Zusammenarbeit sowie ihr Engagement bei der Betreuung der Neutronenstreuexperimente danken.

Außerdem danke ich allen Mitarbeitern des Instituts für Materialphysik: PyuckPa Choi, Daniel Wolde-Giorgis und Dr. Christian Lang danke ich für alles was über den kollegialen Bereich hinausging und das Arbeiten wieder Spaß machen ließ. Für seinen, oft erbetenen, physikalischen Sachverstand sowie viele auch nichtphysikalische Diskussionen (Frauen und Katzen) gilt Dr. Joachim „Jojo“ Bankmann mein aufrichtiger Dank. Prof. Dr. Guido Schmitz, der mir in wissenschaftlichen Gesprächen und praktischen Tipps viel weitergeholfen hat, bin ich sehr dankbar. Allen ,alten“ und „,neuen Kollegen, die jetzt ihren Namen hier nicht lesen, gilt ebenfalls mein Dank für die angenehme und ungezwungene Atmosphäre.

Allen Helfern der Feinmechanik, E-Werkstatt und den Laboranten gilt mein Dank für die unbürokratische und zugleich schnelle Hilfe bei alltäglichen Problemen. Ein spezieller Dank gebührt Tobias Schulz, für seinen unermüdlichen Einsatz in den FIM- Laboren.

Nicht zuletzt möchte ich meiner Frau Anke für ihre Unterstützung danken, auch wenn ich es ihr manchmal nicht einfach gemacht habe. Sie hat mich in Zweifelsfällen immer wieder neu motiviert. Ich danke Dir von ganzem Herzen! 


\section{Lebenslauf}

\section{Persönliche Daten}

$\begin{array}{ll}\text { Name: } & \text { Christian Kluthe } \\ \text { Geburtsort: } & \text { Büren } \\ \text { Geburtsdatum: } & \text { 04. April 1975 } \\ \text { Nationalität: } & \text { deutsch }\end{array}$

Ausbildung

$\begin{array}{lll}\text { Schulbildung } 1994 & \begin{array}{l}\text { Allgemeine Hochschulreife } \\ \text { Mauritius Gymnasium Büren }\end{array}\end{array}$

Wehrdienst $\quad 07 / 94$ bis 06/95 Grundwehrdienst

3. Panzergrenadierbataillon Augustdorf

Studium 10/95 Diplom- Physik Studium an der

Georg- August Universität zu Göttingen

10/97 Vordiplomprüfung Physik

11/99 bis 05/00 Diplomarbeit am Institut für Materialphysik über „Untersuchungen zur innere Oxidation der Legierung Ag-1At.\%Mg mit Hilfe der tomographischen Atomosonde“".

05/00 Diplomprüfung im Fach Physik

seit 06/00 Wissenschaftlicher Mitarbeiter an der Georg- August- Universität zu Göttingen im SFB 602 am Institut für Materialphysik in der Arbeitsgruppe von Prof. Dr. R. Kirchheim. 